\title{
LECTURES ON HEAVY-ION IN-BEAM SPECTROSCOPY
}

R. M. Diamond

Lawrence Berkeley Laboratory

University of California

Berkeley, California 94720

December 1973

\begin{abstract}
This repart was porlCE
sponsored by the prepured is an recount of work

the UnIted by the United States Government. Neither

Conited Sinles nor the United Ststes Arcmir Energy

Commisslon, nor wy of their employets, nor gny of

their contractors, subcontractors, or their employees,

thel any whranty, express or implied, ar sesumes any

pil tibifly or responsibility for the accuracy, com

pleteness or usefolness of any information.

product or process diciosed, or represents, apparatus,

nould not infringa privately, or represents that is tese
\end{abstract}

*

Work supported by the U. S. Atomic Energy Commission.

Lectures presented at the Institute for Nuclear studies Summer School at Dogashima, Japan, August 1972. 
Part I. Angular Momentum Effects on Heavy-Ion Reaction Cross Sections

I shall discuss a, few topics of heavy-ion in-beam spectroscopy. I

There are a rumber of advantages in using heavy ions, but also some disadvantages. In general, there are four features that are emphasized when using heavy ions for in-beam spectroscopy. One is that projectiles have a high nuclear charge. That leads to their almost exclusive use today in Coulomb excitation studies. I shall talk about coulomb excitation in the last two lectures. Another feature that may be emphasized with the use of heavy ions is the possible transfer of a large amount of angular momentum in the reaction. The third feature is that there is a large linear momentum transfer. And a fourth feature is that if you choose the target-projectile nuclei properly, you can get rather good pro-juct specificity when making a particular neutron-deficient nucleus. The last is not an enormous advantage, because if you don't have a great range of particles available to you, you can still make the desired product with protons or alphas. But if you have heavy-ions available and pick the right reaction fusually the first reaction to occur above the coulonb barrier) then usually you can obtain the product more cleanly than is possible with protons. A $\left({ }^{28} 5 i, 4 n\right)$ reaction is cleaner usually than a $(p, 10 n)$ reaction, for example, if you're trying to make a particular product.

In the first two lectures I want to talk about two results of the large angular momentum transfer with heavy ions, and the following two times I'll talk about coulomb excitation. The fact that a heavy ion can bring a large amount of angular momentum to the reaction, whether it makes a compound nucleus or not, influences the results in a number of ways, but 
let me mention two. It can influence the reaction itself, the reaction mechanism. And that, in fact, is the topic I want to take up with you now. But it can al:00 and this is what is more commonly discussed - influenee the nature of the decay of the excited state. For example, if a projectile hits a target nucleus and is. absorbed to form a compound nucleus, it will start the nucleus spinning. Whereever it hits, the nucleus will spın around an axis which is in a plane perpendicular to the beam direction. If there is a large amount of angular momentum transferred, you now have a nucleus with high spin, but the angular momentum is essentially all in the $m=0$ substate. You have a very well-aligned nucleus, and that is of course something that the low-temperature physicists anil chemists work very hard to obtain. As a result of this alignment, one can measure the angular distributions of the de-exciting gamma-rays to learn something about their multipolarities. This is a singles experiment, however, in contrast to a gamma-garma angular correlation one which is a more time-consuming and difficult coincidence experiment. This is an important technique in in-beam spectroscopy. I should also mention that you can perturb that angular distribution with an applied magnetic field and by this means you can measure magnetic moments of excited states. But this topic is not something that is new to you here. Workers at the University of Osaka and Tokyo are among the leaders in this field, so I'm not going to discuss that subject. What I will discuss next cime has to do with the fact that a compound nucleus that has been made by heavy-ion bombardment has a lot of angular momentum and that influences the decay path of the gamma-ray cascade. Predominantly states of high spin are observed, and the cascade goes down fron one state of high spin to the next, picking out preferentially the states of highest spin at 
a given enersy, the so-called "yrast" cascade. Tomorrow I want to discuss examples of such cascades and what they indicate about a new coupling scheme in odd-mass nuclei.

Today we shall discuss ways by which the large amount of angular momentum involved in heavy-ion reactions can influence the reaction mechanisu. Let's consider what happens when we bombard the target nucleus with a beam of projectiles; there are a number of different impact parameters. Some projectiles will be deflecter without touching the nucleus; their distance of closest approach will be tens of Fermis. The process then is mainly elastic (Rutherford) scattering with some Coulomb excitation. - If on the contrary, the impact parameter is small (and the projectile is not at too high an energy) the nucleus can be considered like a black body; it absorbs the projectile and forms a compound nucleus. A particular intermediate case is where the distance of closestapproach is just barely larger than the sum of the nuclear radii. The projectile comes close enough to the target so that just the tails of the nucleon densities overlap and allow transfer reactions to occur, but the trajectory still looks mainly like a coulomb orbit. This is called quasi-elastic scattering. It is harder to exchange many particles so usually one or two particles are transferred. Such direct reactions, and the already mentioned compound-nuclear ones, and inelastic scattering are the main types of reactions possible. Now, let's consider making a conpound nucleus with a very heavy ion. There's a possibility that a very large amount of angular monentum is put into the resulting nucleus if the heavy ion hits at the surface but gets captured. Some years ago, Swiatecki, Cohen, and Plasil discussed a related problem; they calculated the decrease in the fission barrier of a nucleus of given mass with an increase of angular 
momentum. That is, with an increase of engular momentum, the nucleus rotates faster, fission becomes easier, and it flows apart. We can see this from a potential energy diagran. Since the nucleus normally doesn't fission, it has a barrier against fission, when the potential energy is drawn vs. the coordinate for fission, say the distance between the separating fission fragments. Beyond the peak of the barrier is the fall-off due to the coulomb potential. Normaliy the nucleus is sitting in the potential well and cannot ecily penetrate the barrier, so it does not fission. But that is for angular momentum zero. If we now ratate this nucleus, we must add a centrifugal potential, $\left(h^{2} / 2 J\right) I(I+1)$ : This falls very steeply with distance (assuming $\widetilde{S}_{\propto} x^{2}$, for example) and so if the angular momentum is large cnough, the well and barrier shrink to an inflection point; the fission barrier goes to zero. In the time it takes for particles to traverse the nuclear dimension, something in the order of $10^{-22}$ seconds, the nucleus blows apart. If that idea is valid, then you might expect with a heavy-ion reaction where a large ampunt of angular momentum can be brought in, the compound nucleus may not always form. The compound-nuclear cross section would not go up as rapidly with energy as the total reaction cross section. And that is shown in Fig. 1-1 where the cross section is plotted as a function of bombarding energy for neon and oxygen on alumin'm. The total reaction cross section has actually been measured for the case of oxygen on aluminum by wilkins and Igo, and up to $165 \mathrm{MeV}$ oxygen it is increasing, approaching $\sim 1 \frac{1}{2}$ barns at that energy. But the experiment of Kowalski, Jodogne, and Miller just a few years ago, shows that the compound-nuclear cross section, as measured by counting the tracks of the recoiling compound nuclei in a mica or plastic detector, falls with 
increasing energy above a certain value. In fact, with $160 \mathrm{MeV}$ oxygen or $200 \mathrm{MeV}$ neon on aluminum, the compound-nuclear cross section is only about one-fourth or one-fifth of the reaction cross section, only $300-400 \mathrm{mb}$ of the $13-2 b$ total reattion cross section.

So the problem, an interesting problem of only the lasc few years, is where is the missing cross section? It is not in compound-nucleus fornation; it is not in inelastic scattering. It might be in quasi-elastic scattering, in one-, two-, three-particle transfer reactions in which the (modified) projectile goes off near the limiting elastic angle.' It might be that in a still closer collision a new type of process occurs. This is not a new idea; it was suggested more than ten years ago by Wolfgang and kaufmann when they first started working wi th heavy-ion beams at the Yale Hilac. They talked of a "grazing" reaction where the nuclei barely touch as they pass, and so the nuclear interactions come into play for a short time. The path, the trajectory, gets deflected forward, because the nuclear interaction is attractive, and nucleons can be exchanged during the collision. So a multinucleon transfer occurs, but it is not an increase in the quasielastic transfer cross section because it does not go on a Rutherford trajectory, but at a more forward angle. Relatively many particles may transfer, because the nuclei actually make contact, but the contact is not strong enough to permit them to hold together, to form a compound nucleus. There have been two ways to stuay this problem (in the last few years there have been a number of studies). Wolfgang and Raufmann did it by radiochemical methods. They used thin targets, and so let the nuclei recoil out of the target into thin catcher foils. After irradiation, the catcher foils were dissolved, the 
different elements were separated chemically, and then counted. With hemispherical catcher foils, it was possible to measure angular distributions of the products, and with stacked foils range measurements were obtained, so they got a lot of information. But it is a lot of work and tine-consuming. In the last three or four years, counter telescopes have been used to look at the scattered projectile, but not the recoiling product. Again, a great deal of infomation is obtalned, but there are some difficulties with this, too, because the cross sections may vary markejiy with the angle of the scattered particles. It becomes especially difficult to use a counter telescope at small angles where you get wiped out by the beam itself. And yet, measurements must be made to near $0^{\circ}$ if thers is appreciable cross section at sn..1] forward angles. Magnetic spectrometers can do this, they have only come into use very recently, and it is still an extremely time-consuming experiment because you have to measure many angles with good statistics, and do this at several bombarding energies. This may require weeks of running time, and that is not available to most groups. So, in fact, very complete studies have not been made - it is just too time-consuming. However, a lot of information comes from such studies, because you obtain not only the total excitation function and angular distribution, but those to individual states of the taxget and projectile. That is, there may be structure in the projectile spectra, and that tells you something about the states you are producing in the projectile and in the target. There may also be a continuur; usually there are both. Our idea was to usc the technique of gamma-ray spectroscopy and merely look at the target with Ge counters - look at the gamma-rays coming out of the target - both in-beam and between beam bursts (the Hilac beam is pulsed 
so that we have $\sim 5 \mathrm{~ms}$ on and $\sim 23$ ms off). The work was done mainly by Dr. Falk Pühlhofer, who was with us for half a year. If such work is done in a selected region of the periodic table, you can identify the nuclides by their gamma-ray spectra alone, and this method has the advantage that it is extremely quick. The total bombardment time for the experiments described below was about ten hours. Only one or two angles need be done, because, roughly speaking, the gamma-ray angular distributions are isotropic; their anisotropy is not very large, about 30-40\%. That can be taksn into account, and even if done roughly, the error in the cross section is much less. So a number of bombarding energies can be run through in a few hours to get relative cross sections. But the results are cross sections for production of a particular ganma ray, not of that nuclide. That is an important distinction; and they are not the same whing, of course. If the nucleus is made in its ground state, you don't see it by this method. or, if it is made in a very highly excited state which decays to the ground state with a very highenergy transition, it may not register. in the germenium counter, especially if you use small counters as we did at first. The method is complimentary to the particle experiments but in no way supplants them. You just don't get enough information. But it is a nice start - it gives some information guickly. The systems we chose to study had aluminum as a target and ${ }^{20} \mathrm{Ne},{ }^{16} \mathrm{O}$, and ${ }^{12} \mathrm{c}$ projectiles. These were piuked because there already were some data on the ${ }^{16} \mathrm{O}$ and ${ }^{20} \mathrm{Ne}$ systems, the resulting gamma-ray transitions were fairly unique, and such light systeme would magnify the effect of large amounts of angular momentum brought in. 
We can obtain information on the mechanism of production of the product nucliāes by determining how much linear momentum is given them. If a compourd nucleus is made by bombarding aluminum with neon, the resulting compound nucleus is only a little over twice the mass of the original projectjle, so there is a large center-of-mass motion, a large linear momentum transfer to the product nucleus. If, on the other hand, a compound nucleus is rut made but a direct reaction", such as a transfer reacticn, occurs, then the projectile itself continues with essentially the same velocity it had to start with. That is, the projectile has a large final momentum, but the target receives very little momentum, so it stays put. We want to be able to distinguish between these two reaction mechanisms in our gamma-ray studies, and there are two ways to do that which are complimentary. One method is to use targets of varied thickness. When a target foil is thin compared to the recoil range of the compound nucleus, the compound nucleus recoils out of the target. If the gama-ray life-time is large compared to nanoseconds, the recoiling nucleus will go quite a few centimeters, before it emits the gamma ray. Then our gamma counters close to the target may not detect it. If the target is made thicker, we may still not detect it. But at some point the farget becomes thick enoigh so that a compound nucleus formed at the front of the target has its entire range insile the target; it stops in the target. The detector will see it, and as the target is made still thicker, we shall see an increase in the yield from then on. A plot of gama-ray yield against target thickness for a compound-nuclear-

*) I'll use the term direct reaction or non-equilibrium reaction to mean any reaction that does not go through a compound nucleus. There is not uniformity in the literature on this teminology, so this definition is valid for this talk, but not generally in the literature. 
reaction will show nothing up to a thickness equal to the recoil range, and then the yield will go up linearly. The range for the compound-nucleus in the reactions I shall describe below is $\sim 4 \frac{4}{2} \mathrm{mg} / \mathrm{cm}^{2}$. Even the use of as few as three target thicknesses will give an estimate of whether there has been a full-momentum transfer, a compound-nuclear reaction, or not. For if the gamma ray comes from the nucleus derived from the targ̣et in a direct reaction, that is, from a product with low linear momentum, the yield curve will rise linearly with the target thickness starting essentially at zero thickness. These two cases are shown schenatically in Fig. 1-2. That is a way of distinguishing a high-momentum transfer-product nucleus from a low-momentum one if the life-time of the gamma ray is long compared to nanoseconds. If the life-time is very short compared to nanoseconds, we can see whether the gamma-ray is Doppler-shifted. It will show a Doppler-shift if the detector is at a forward angle, and even at $90^{\circ}$ it will be brcadened because the counter has an appreciable solid angle. There are three possible cases. If the gamma-ray comes from either a compound nucleus or a product derived from the projectile, there will be a Doppler-shift. If it comes from a product derived from the target in a direct reaction, there would be no Dopplermshift or broadening. So again by this method you can distinguish whether the garma-emitting fragment had a large velocity or not.

well, that's the idea; it is a simple experiment. The work is mainly in analyzing the data. You must go through the reams of spectra and identify the gamma rays, correct their intensities for the detector efficiency, and so obtain absolute values for the cross sections to produce them. Some 30 
gamme rays from 22 nuclides were identified in the reactions of ${ }^{20} \mathrm{Ne}$ and $16_{\mathrm{O}}$ on $27 \mathrm{Al}$, and Fig. $1-3$ gives examples of the spectra. The top two figures are Ne on $A I$ and the bottom cne is $C$ on $A l$. Note that there are a finite number of gamua-ray lines, indicating the experiment can be done, which is the first thing we had to convince ourselves. Almost all of the major gamma-rays can be identified. We look separately both in-beam and out-of-becm, and some of the useful data comes from the out-of-beam spectra which are gamma-rays following $\beta$-decay and give the cumulative mass-chain yield, But remember we are not observing the yield of a nuclide, we are observing the yield of the gamma-ray. This gives only a lower limit on the yield of the nuclide. Neon ions of $85 \mathrm{MeV}$ energy on aluminum are at a reasonably low energy, so one can expect mainly compound-nuclear reactions, and that is what is observed. Argon 38 and ${ }^{42,43} \mathrm{Ca}, 42,43$ Sc are all compound-nuclear products, as will be indicated below from target thickness vs. yield curves and also fron the excitation..functions. At higher bombarding energies, the yields of these particular nuclides decrease and other compound-nuclear products appear that involve the evaporation of more particles. For example, ${ }^{32} \mathrm{p}$ shows up as a reaction product at higher energies. But with the highest energy 20 Ne beam, $200 \mathrm{MeV}{ }^{20} \mathrm{Ne}$ on ${ }^{27} \mathrm{Al}$, a number of products such as ${ }^{18,19} \mathrm{~F}$, $19,21 \mathrm{Ne}, 22,24 \mathrm{Na}, 25_{\mathrm{Mg}}$, and $26,28 \mathrm{Al}$ appear, all in the mass range 18 to 28 . As you will see below, all of these are low-momentum products derived from the target in direct reactions. It may not be obvious from Fig. 1-3, so I would like to point out that the relative yields of ${ }^{18,19} \mathrm{~F}$ and other products in the mass range 18 to 28 have almost precisely the same relative yields for $200 \mathrm{MeV}{ }^{20} \mathrm{Ne}$ as for $160 \mathrm{MeV}{ }^{16} 0$ on aluminum, and for $120 \mathrm{MeV}$ carbon on aluminum. 
This is not true, of course, for the compound-nuclear products, which show very different yields depending on what the projectile is (and the energy). Another point to be noted is that the peak of the ${ }^{21} \mathrm{Ne} 351 \mathrm{keV}$ transition is broad at the base for the $200 \mathrm{MeV}{ }^{20} \mathrm{Ne}$ irradiation. Most of the yield, in fact, is in the Dopplex-broadened peak and not in the small spike. But that same transition in $21_{\mathrm{Ne}}$ is not broadened in the spectrum from $160 \mathrm{MeV}$ ${ }^{16} 0$. This illustrates that there are two mechanisms operating to make the ${ }^{21} \mathrm{Ne}$, one involving a large linear momentum transfer, the other a small momentum transfer. with ${ }^{16} 0$ only the latter occurs, so the ${ }^{2 I_{\text {Ne }}}$ in this case is derived from the target. With ${ }^{20}$ Ne as the projectile, it can also be made by neutron pick-up.

Figure 1-4 shows the region of the periodic table involved. The target, projectile, and compound nucleus are marked and the 22 product nuclei identified are shown. The minus signs indicate nuclides which have ganma-rays which we should have seen if they had been made in any reasonable yield. A reasonable yield, with this technique, means millibarns. In-beam gamma-ray spectroscopy is not a very sensitive technique. The blank squares are the nuclei about which we know nothing. We did not see them, but there is some question whether we should have seen them because of their location or because of the nature of tiieir decay scheme. Clearly the nuclei we did observe fall near the valley of stability. That is not too surprising, since it rises rather steeply on each side in light nuclides and so one cannot move very far from it with any appreciable cross section. However, most of the nuclides seen appear to fall into two groups. One goes from the compound-nucleus down to ${ }^{32} \mathrm{p}$, and the other group involves masses from 28-18. The first group, as shown below, are 
compound-nuclear products. The second group, with the exception of ${ }^{19} \mathrm{~F}$ and part of the ${ }^{21}$ Ne yield, come from the target nucleus by direct reactions, or gerhaps more accurately, by ton-compound-nuclear reactions. The singleparticle transfer reactions leading to ${ }^{19} \mathrm{~F}$ and ${ }^{21} \mathrm{Ne}$ have the biggest cross sections in this group, but there is a non-legligible cross section for all the other mass 28-18 nuclei, and one of the problens is how do they come about - what are they? Before we come to some speculation about chem, let me point out one more feature on this figure which is rather striking. That is the asymetry in yield between the product nuclei of mass $28-18$ coming from the target, ${ }^{27} \mathrm{Al}$, and the lack of observation of direct reaction products of correspondingly heavier mass also coming from the target. For example, if the mass 28-18 group are the result of multi-nucleon transfer, multi-nucleon transfer reactions should also be able to transfer up to 9 particles into the target and so give heavier products. Now we haven't proved that they are not made, beciuse as I mentioned earlier, we dun't really know that most of the direct-reaction produzts in the mass range $28-38$ are not observed because of some quirk of their decay schemes. But the indication is that there is a marked asymmetry, and that is a clue, of course, as to what is happening.

Figure 1-5 gives some examples of the plots of gamma-ray yield vs. target thickness. These help decide whether the transitions come from nuclei with a large velocity or not. The unshifted peak in ${ }^{38}$ Ar is typical of nuclides made from compound-nuclear reactions. Because they recoil out of the thin ierget, they cannot be seen unti? the target thickness is greater than the recoil range ( $4 \frac{1}{2} \mathrm{mg} / \mathrm{cm}^{2}$ ). Phosphorus 32 shows the same behavior. So these 
nuclides, we think, are made with a large momentum transfer by compoundnuclear reactions, and their excitation functions indicate the same thing, as you shall see. The other nuclides, particularly those from mass 28-18, show essentially straight lines from the origin for such plots of yield against target thickness, and that indicates that they are made with little momentum transfer, i.e., they are direct-reaction products from the target. Figure 1-6 shows some excitation functions. These are nct very good since they have only four points, but we had very limited machine time before it shut down. Again the heavier nuclei, ${ }^{38} \mathrm{Ar},{ }^{36} \mathrm{Cl}$, and ${ }^{32} \mathrm{P}$ are indicated to be compound-nuclear reaction products; they show maximum cross sections and thresholds exactly where you would calculate them for compound-nuclear reactions, if the particles being emitted are considered as alpha particles whenever two protons and two neutrons are evaporated and the rest as individual nucleons. That would give thresholds where the arrows indicate. The yield of ${ }^{38}{ }_{\text {Ar }}$ appears to consist of two reactions; the lowest energy one involves two alphas and a proton evaporating, and then at higher energies, one alpha and five nucleons came out. Now the other examples, the nuclei with masses from 18-28, all show about the same behavior. Almost all show a peak with a threshold around $100 \mathrm{MeV}$. In scme cases that is 50 or $60 \mathrm{MeV}$ below the compound-nuclear threshold, even for alpha-particle evaporation. For example, to make ${ }^{18_{F}}$ the compound nuclear threshold is about $160 \mathrm{MeV}$, and the observed threshold is below $100 \mathrm{MeV}$. So I am pretty sure these are not compound-nuclear reactions and that agrees, of course, with the evidence that they come from a target nucleus with little momentum transfer. 
I might mention that the same sort of experiments were done with oxygen on aluminum with the same sort of results, although they are not quite as nice nor as complete. With oxygen on aluminum the thresholds for these reactions seen to be about $120 \mathrm{MeV}$, a little higher in energy and they are not quite so well marked. But again they cannot be ascribed to compoundnuclear reactions.

So, what do we have? We know that the total reaction cross section goes up monotonically, as is shom in Fig. $1-7$ for ${ }^{20} \mathrm{Ne}$ on ${ }^{27} \mathrm{Al}$. It starts rising at the coulomb barrier, about $40 \mathrm{MeV}$. The compound-nuclear reaction cross section determinations of Kowalski et al. give the points indicated, showing a cross section decreasing from about $90 \mathrm{MeV}$ on. And we have found that the direct reactions which lead to the products derived from the target nucleus, ranging from ${ }^{18}$ to to ${ }^{28} \mathrm{Al}$, all start up around $100 \mathrm{MeV}$. I have not mentioned one particular reaction which I should, and that is fission. In particularr, compound-nuclear fission. The people who did the experiments to determine compound-nuclear cross sections looked at the recoil of the compound-nuclear products into track plates; the latter are purposely chosen so they will not Iespond to masses of the order of those of the projectile. For example, for neon on aluminum they will not respond to masses of the order of 20 or less. Only heavier nuclei, corresponding to products derived from the compouna nucleus, ${ }^{47} \mathrm{~V}$, will make tracks, say masses in the range from 30-47. But that means if symetric fission occurs it would not have been seen, because the fission fragments would be back in the range of 20 plus mass units. So, in fact, their cross sections may be too small by however much there is of 
compound-nuclear fission. What if all the products we abserved in the mass range from 18 to 28 were the results of compount-nuclear fission? I cannot definitely say no, that is not so, but there are a number of plausibility arguments against it. Fission from a compound nucleus has a certain angular distribution; ic has to be symmetric around $90^{\circ}$ in the $c$. of $\mathrm{m}$. system. There has to be as many of a given fragment in the backward direction as in the forward direction. In the laboratory system with ${ }^{20} \mathrm{Ne}$ on ${ }^{27} \mathrm{Al}$, the forward direction means going forward with a velocity about that of the original projectile, and the backward direction means almost tanding still. (Exactly so if the target and projectile were the same mass and the same as the symuetric fission products.) But that means we should see all short-lived Y-ray transitions equally likely with Doppler-shift and without, because some come from stationary fragments and some come from forward-moving fragments. In the case of the $351 \mathrm{keV}$, transition in ${ }^{21} \mathrm{Ne}$ made with ${ }^{16} \mathrm{o}$ on ${ }^{27} \mathrm{Al}$, there is no Dopplerbroadened component. There should have been, if this product were the result cf compound-nuclear fission. Two other high-energy gamma rays, seen only with the coaxial counter, also do not show equal amounts of broad and narrow peaks. But these are not many cases, so it is not a very strong argment. Another argument, though again not a very strong one, is the feature mentioned earlier that the relative yields of the products from ${ }^{18} \mathrm{~F}$ to ${ }^{28} \mathrm{Al}$ were the same whether aluminum was bombarded with ${ }^{20} \mathrm{Ne}$ or ${ }^{16} \mathrm{O}$ or ${ }^{12} \mathrm{C}$. clearly, very different compound nuclei are made, ranging from ${ }^{47} \mathrm{v}$ to ${ }^{39} \mathrm{~K}$, so you might expect the fission fragments to show a distribution which would vary. That is, if they showed any probability of seaking around symmetric fission, then obviously that peak would move down 4 mass units and should be noticeable in the results. 
This did not appear to happen. Of course, the answer to that could be that fission in such light fragments under these conditions can cover a broad range of mass distributions with equal probability; there is no symetric peaking but fragments from mass 1 to mass 47 sccur with almost equal probability. To tiat I can answer that we should then see everything from mass 1 to mass 47 , and we do not. But there surely has to be some fission, and we can set a rough limit. We will not see any product with a cross section less than a few millibarns, so if a hundred products are possible from the mass 47 compound nucleus, each requiring a few millibarns, the total cross section could be a few hundred millibarns. That is, anywhere from zero to, say, 300 millibarns could go into compound-nuclear fission. Thus, that adds from zero to a few lundred millibarns to the 300-4v0 millibarns of compount-nuclear cross section already observed by the track-plate investigators for $200 \mathrm{MeV}{ }^{20} \mathrm{Ne}$ or ${ }^{27} \mathrm{Al}$, so let's double their number and consider $700 \mathrm{mb}$ an upper limit for the campound-nuclear cross section. That is $\mathrm{s}^{+}$ill less than half the total reaction cross section of $\sim 1.7$ barns. So there is still of the order of a barn to account for.

Let us consider the calculation of reaction cross sections more quantitatively using a simple sharp-cutoff model. The reaction cross section, if you consider the target a black sphere with plane waves incident on it, is

$$
\begin{aligned}
& \sigma_{R}=\pi \hbar^{2} \sum_{L=0}^{L_{\max }}(2 L+1)=\pi R^{2}\left(I-V_{C B} / E\right) \\
& \left.L_{\text {max }}=K_{C B} R, R=r_{0}\left(A_{1}^{1 / 3}+A_{2}^{1 / 3}\right)+t, K_{C B}=\sqrt{2 \mu\left(E-V_{C B}\right.}\right) / \hbar
\end{aligned}
$$

where $A_{1}, A_{2}$ are the mass numbers of the projectile and target, respectively, $\mu$ is the reduced mass, $\lambda$ the wave length, $E$ the c.m. energy, and $v_{C B}$ the Coulomb barrier. Tie reaction cross section is calculated assuming that all collisions in which the distance of closest approach is less than $\mathrm{R}$ lead to reactions. For the radius 
parameter, $r_{0}$, a value of $1.16 \mathrm{fm}$ was taken. The parameter $t$, which accounts for the diffuseness and the range of nuclear forces, was determined from a fit of the measured reaction cross sections for ${ }^{16} \sigma_{0}$ on ${ }^{27} A 1$ to be $t=2.0 \mathrm{fm}$. $\sigma_{R}$ starts up at the coulomb barrier, and goes to the geometric linit of $\pi R^{2}$ at high bombarding energies as drawn in Fig. 1-7. The limiting angular momentum, $L_{\text {max }}$ is the angular momentum that corresponds to hitting the edge of the target and is given in Eq. (1-2). For $200 \mathrm{MeV}$ neon on aluminum, the value calculated for $L_{\max }$ is $60 \mathrm{~h}$. Equation (1-3) proviaes the same sort of calculation for the compount-nuclear cross sections.

$$
\sigma_{C N}=\pi \hbar^{2} \sum_{L=0}^{L_{c}}(2 L+1)= \begin{cases}\sigma_{R} & \text { for } L_{c r} \geqslant L_{\max } \\ \pi \hbar^{2} L_{c r}\left(L_{c r}+1\right) & \text { for } L_{c r}<I_{\max }\end{cases}
$$

with $\mathrm{L}_{c}=$ minimum ( $\mathrm{L}_{\mathrm{er}}, \mathrm{L}_{\max }$ ). The compound-nuclear cross section will be equal to the total reaction cross section if the critical angular momentum (that angular momentum at which the Coulomb repulsion and centrifugal forces overcome the nuclear surface tension and allow the nucleus to blow apart) is greater than $L_{\max }$ of the reaction. That is, the target is a black body and anything that hits it is caught and forms a compound nucle' as long as the angular momentum brought in is less than the critical angulaz momentum. But if the critical angular momentum is lower than $L_{\text {max }}$ all the particles bringing in more angulax momentum than $L_{c r}$ will not form a compound nucleus. The critical angular momentum cannot be calculated from any simple formula, but if you have, as we do, a w. Swiatecki at your place, you ask him to calculate it from the liquid-drop model. He does this by calculating how much angular momentum is needed to reduce the fission barrier to zero. An interesting feature is that we are getting to a point where the rotational energy is becoming comparable in magnitude to the Coulomb energy and the surface tension energy r.f the liquid drop. That is a new regime in nuclear physics. 
The critical angular momentum on such a plot as Fig. 1-7 will be a hyperbola, as the cross section for any constant value of $L$ goes as $\hbar^{2}$ or $1 /$ E. The critical angular momentum for the compound nucleus ${ }^{47} \mathrm{~V}$ is $45 \mathrm{~h}$, as calculated by the liquiddrop model. It is less than $L_{\text {max }}$ at $200 \mathrm{MeV}{ }^{20} \mathrm{Ne}(60 \mathrm{~h})$, and so it would be expected that only about one-half the total cross section would go into compoundnucleus formation at this energy, if the liquid-drop model picture is right. And let me emphasize, that is a model calculation and may or may not have anything to do with reality. At the end I shall point out a somewhat different qualitative approach to this problem. But this calculated value for $L_{c r}, 45 \mathrm{~h}$, is still rather large compared to the experimental results for compound-nuclear cross sections, as they indicate a value of about $30 \mathrm{~h}$ (see Fig. 1-7). However, a correction has to be made to each value. The experimental curve does not include compound-nuclear fission, and any such fission would raise the value of $30 \mathrm{~h}$. And the calculated value is too large because the nucleus will still blow apart, rather than form a compound nucleus, even with a small, non-zero, fission barrier. So Swiatecki calculated the angular momentum at which the fission barrier is not zero but is equal to the energy it takes to evaporate a particle, a nautron or proton, whichever is most likely to evaporate from this compound nucleus. (That is a proton in this case. The coulomb barrier is low, and the proton binding energy is lower than the neutron binding energy.) He calculated the angular momentum corresponding to a fission barrier, not of zero, but $\sim 9 \mathrm{MeV}$, and came back with the answer $30 \mathrm{~h}$. I should point out that he did not know the experimental results, so that is pretty good agreement, but somewhat fortuitous, of course, since both experiment and calculation are only good to several units in h. But it shows that such calculations are in the right ball park.

We have one more number to add to this. We have abserved a number of products, which I have called direct-reaction or non-equilibrium products coming from the target, all of which have excitation functions starting up at 
about $100 \mathrm{MeV}$. What part of the missing cross secticn do they represent? Since we do not know the total absolute cross section for all such products, but only for the ones we have seen, we shall have to consider the shape of their excitation functions as being typical, namely that they start having measureable cross sectiors above about $95 \mathrm{MeV}{ }^{20} \mathrm{Ne}$ bombarding energy. This threshold corresponds to an angular momentum limit of about $35 \mathrm{~h}$. What does this mean? We can only speculate. A value of $35 \mathrm{~h}$ might not be an unreasonable limit for the compound-nuclear cross section including compound-nuclear fission (corresponding to about 200 millibarns fission). But how much cross section have we actually observed in the direct or non-equilibrium type reactions, as Fig. 1-7 shows that at $200 \mathrm{MeV}^{20} \mathrm{Ne}$ the total amount of cross section for $\sigma_{R}-\sigma_{C N}$ corresponding to a $35 \hbar$ limit on $\sigma_{C N}$ is more than $1 \mathrm{~b}$. We can integrate the yields obtained from the products from ${ }^{18} \mathrm{~F}$ to ${ }^{28} \mathrm{Si}$ and we come up with about 300 millibarns cross section. About half of that is in the one-particle transfer reactions to make ${ }^{19} \mathrm{~F}$ and ${ }^{21} \mathrm{Ne}$. But we did not see ${ }^{21} \mathrm{Na}$ and ${ }^{19} \mathrm{Ne}$, so if they are equally produced, the cross section should be doubled. In fact, I think they should be larger than that because we do not observe any cross section directly to the ground states, we to not observe any cross section for a projectile-derived product that decays by a gammaray transition with a life-time greater than nanoseconds. We do not observe high-energy gamma-rays that come from high-lying states and go atraight to ground because, unfortunately, most of these measinements were made with a small planar detector. We used a coarial detector only in the last run, and we did observe additional 1 to $2 \mathrm{MeV}$ gamma-ray transitions. So, the fact that we have only measured $300 \mathrm{millibarns}$ cross section does not seen too bad. I believe that the cross section for these reactions could easily be twice that 
figure. However, I am a little bit worried about how to get that number up to 1 barn which is about what we are missing.

Let us look at this situation in a slightly different way. What are the types of reactions we are considering? The projectiles that hit the target at not too high an energy are absorbed to form a compound nucleus. If they hit off center, there is a large amount of angular momentum transferred. The orojectile sticks to the target, a neck forms between them, and while they rotate around, the neck gets larger until they merge into a single compound nucleus.

The other extreme situation is where the projectile goes by and does not quite touch, "quasielastic" scattering. One or two nucleons car be exchanged, but not very many because there is too much distance between nuclei. The projectile trajectory is essentially that of an Elastic scattering. Finaliy, the reactions we want to talk about, the grazing reactions, or the direct reactions, or the non-equilibriurn reactions (and you'll see why I use that term) are the ones in-between. The projectile actually hits on the surface. If the energy is high enough, it will hit and start to form a neck, but as it rotates around the target the following forces are acting on it. The attractive nuclear force is pulling it in to amalgamate with the target. The coulomb repulsion is trying to separate the two nuclei, to blow them apart. And finally, the linear velocity of the projectije is initially pushing the projectile into the target, holding them together and letting the neck form. But when the two nuclei have rotated at most $90^{\circ}$, the motion of the projectile is now away from the target, with the coulomb repulsion and against the nuclear attraction. If the original velocity is high enough, 
there is going to be a reasonable chance that the projectile goes off - it does not stick. If the original velocity is not too high, the projectile will continue around the target, the neck will grow bigger and bigger, and it will form a compound nucleus. And there is going to be every gradation in-between. What happens to the projectiles that pull off? They have rotated some small angle around the target, so, clearly, they will not show a quasielastic pattern. The nuclear interactıon has deflected them from that path, pulled them forward. They will appear in a cone at smaller forward angles than the "critical" angle for quasielastic scattering. And since they have been in close contact with the target for a fraction of a nuclear rotation, they can have exchanged nucleons with it. Multinucleon transfers are quite possible, but the collision is a very drastic, a traumatic, experience for the two nuclei to undergo. They hit very hard, but not at a high enough relative velocity to go through each other. (A 100 MeV proton goes through a nucleus, but these projectiles are at 5-10 MeV per nucleon, sc they cannot go through the target.) They have to go around each other on the surface. In so doing, they are obviously heating up that surface. There is a large amount of frictional energy expended. In the process, small pieces of nuclear matter are chipped off and sent forward, namely protons, alphas, and neutrons. Actually, the protons and alphas were observed ten years ago in early experiments at the Yale Hilac; there is a large cross section for producing protons and alphas at small scattering angles from this kind of reaction. It was not quite understood why at that time, but it was obvious they had to cone from some splitting up of the projectile and/or target. The resulting products of both the projectile and the target are thus going to be 
smaller in mass, and this will be accentuated by the evaporation of still more nucleons by the local heating. There is a reasonable consistency to this picture, because it helps explain why we see products below che target more readily than those above. Products derived from the projectile in such collisions should also favor smaller masses and should be predominantly at forward angles. They also should have their linear velocity reduced, because a lot of energy was used up against friction and in breaking-off these small fragments. That is exactly what has been observed in the experinents, for example, at Orsay by Prof. Lefort's group. However, this picture does not explain everything; it is merely a suggestion, not a fact. What are the possible consequences? First of all, it is clear that with the use of very heavy ions and we hope to be working with very heavy ions in the future - chere may be a lot of trouble seeing compound-nuclear reactions. In fact, I would guess that to make compound-nuclear products, argon is as big a projectile as you want to use. For larger ions the cross section may get so small that it will not do you any good. That is very serious, of course, for the people who want to make super-heavy elements. They are going to have real trouble making them by compound-nuclear reactions with very heavy projectiles, and they are well aware of this. The cross sections do seem to be getting lower and lower as more work is done with such ions. Net a lot is gained by going to transfer reactions, because the multinucleon transfer reaction is the only way to make big changes and they tend to go preferentially one way, to smaller products. (I don't think they are all multinucleon transfer reactions in the literal sense, because I do not think they are all two-body reactions, blit three-body, and many-body reactions.) The particles iost from the ${ }^{27}$ Al target when 
bombarded with 20 ne $\equiv r=$ not all picked up by the Ne; many of them are lost. That is not to say that there are no multinucleon transfer reactions. There are, but the cross sections are quite small. I do not think you are going to see several hundred millibarns in nultinucleon transfer reactions. But I think that much of the missing cross section may well be in reactions like those I have just described, where the nuclei hit but do not stick to form a compound nucleus. Energy is lost against friction as the two nuclei roll around each other, and energy and nucleons can be interchanged between them, but they separate before any equilibrium is achieved. That is why I tend to call them non-equilibrium reactions. We have not proved that they exist. This is only a suggestion, a hypothesis. It is quite obvious that many more experiments must be done to settle the nature of the reaction mechanisms and to account for the unknown cross section. 
Part I

Figure Captions

Fig. 1-1. Compound-nuclear cross section vs. beam energy for ${ }^{20} \mathrm{Ne}$ (upper curve) and ${ }^{16} 0$ (lower curve) on 27 .

Fig. 1-2. Gamma-ray intensity as a function of target thickness. Case 1: $Y-r a y s$ from nuclei formed from the target in a direct reaction (no momentum transfer). Case 2: $\gamma$-rays from nuclei formed in compound-nucleus reactions (full momentum transfer); this curve holds for nuclei which are stopped in the target. Gamma rays from recoiling nuclei are either absent, if the lifetime is much larger than $l \mathrm{~ns}$, or they can be distinguished by the Doppler effect. A narrow range distribution is assumed.

Fig. 1-3. Gamue-ray spectra obtained in the ${ }^{20} \mathrm{Ne}$ and ${ }^{16} 0$ bombardments of $27 \mathrm{Al}$. Fig. 1-4. Reaction products identified in the experiment with the ${ }^{20}$ Ne beam. Projectile, target and compound-nucleus are indicated by dark squares. A minus sign indicates that a nucleus has not been observed although it should be detectable in this experiment.

Fig. 1-5. Experimental yield functions for $206 \mathrm{MeV}{ }^{20} \mathrm{Ne}$ on ${ }^{27} \mathrm{Al}$. A correction for the energy loss in the thickest target has been included. The energy of the transition and the lifetime are indicated.

Fig. 1-6. Excitation functions measured for ${ }^{20} \mathrm{Ne}$ on ${ }^{27} \mathrm{Al}$. The arrows labeled CN indicate the calculated threshold for compound-nucleus reactions (with emission of nucleons and a-particles). Error bars include only statistics and errors due to background subtraction. 
Fig. 1-7. Energy dependence of the reaction cross section, $\sigma_{R^{\prime}}$ and of the total compound-nucleus cross section, $\sigma_{\mathrm{ON}}$, for ${ }^{20} \mathrm{Ne}$ on ${ }^{27} \mathrm{Al}$ according to the sharp-cutoff model described in the text. The curve parameter is L cr, the upper limit of the angular momentum of the compound-nucleus. The data points for $\sigma_{C N}$ are from L. Kowalski, J. C. Jodogne, and J. M. Miller, Phys. Rev. 169 (1968) 894. 


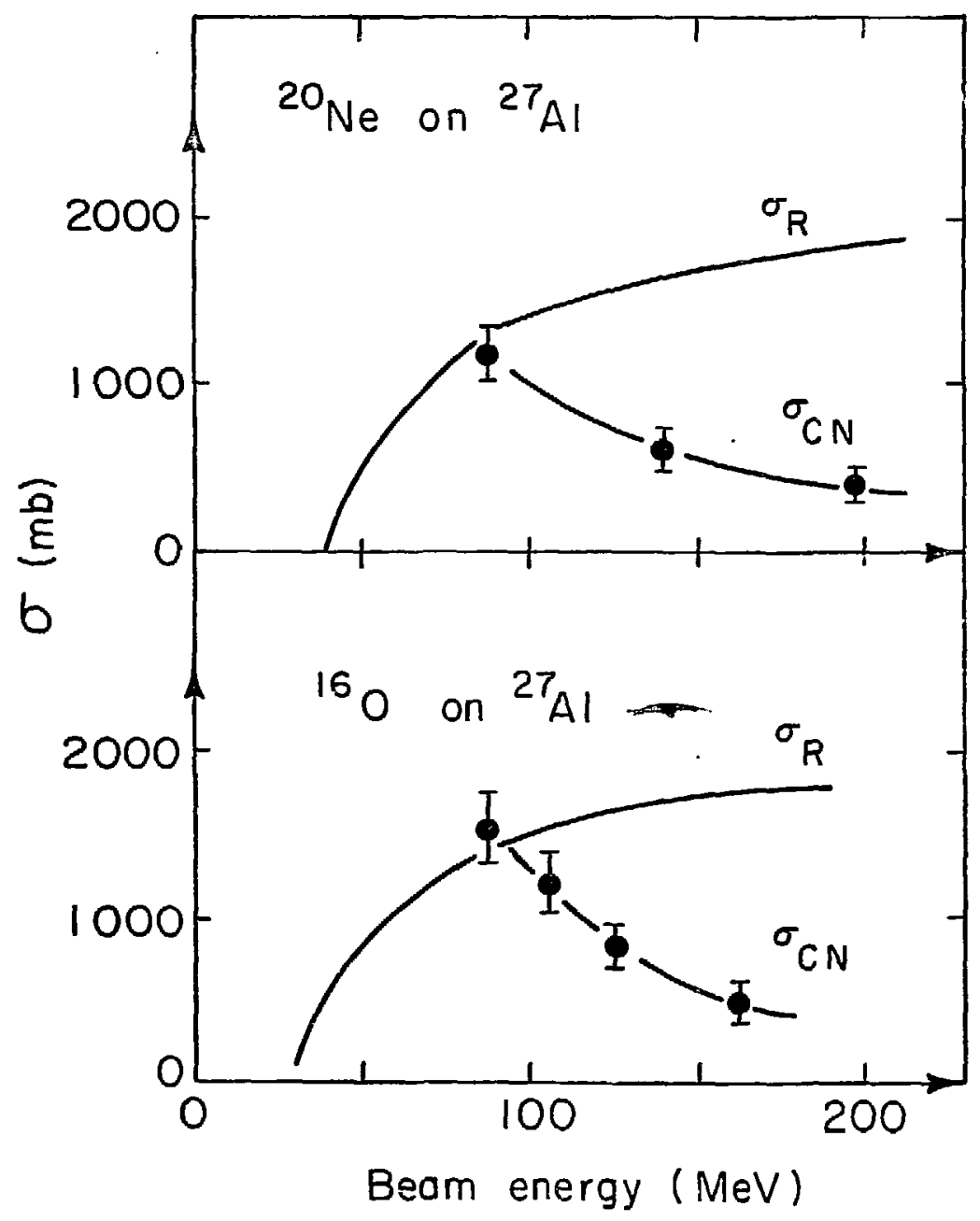

$X B L 7110-4533$ 


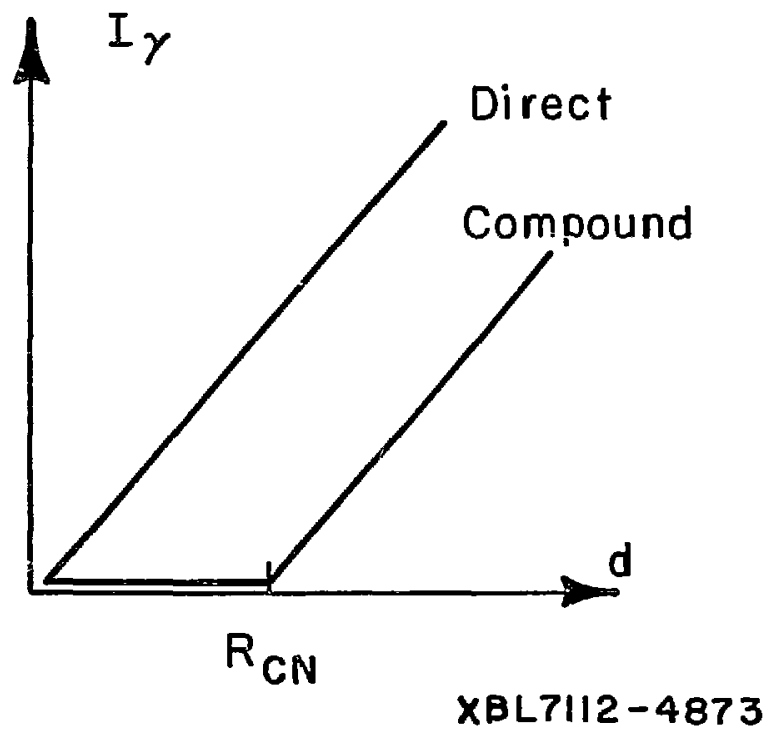

Fig. 1-2 


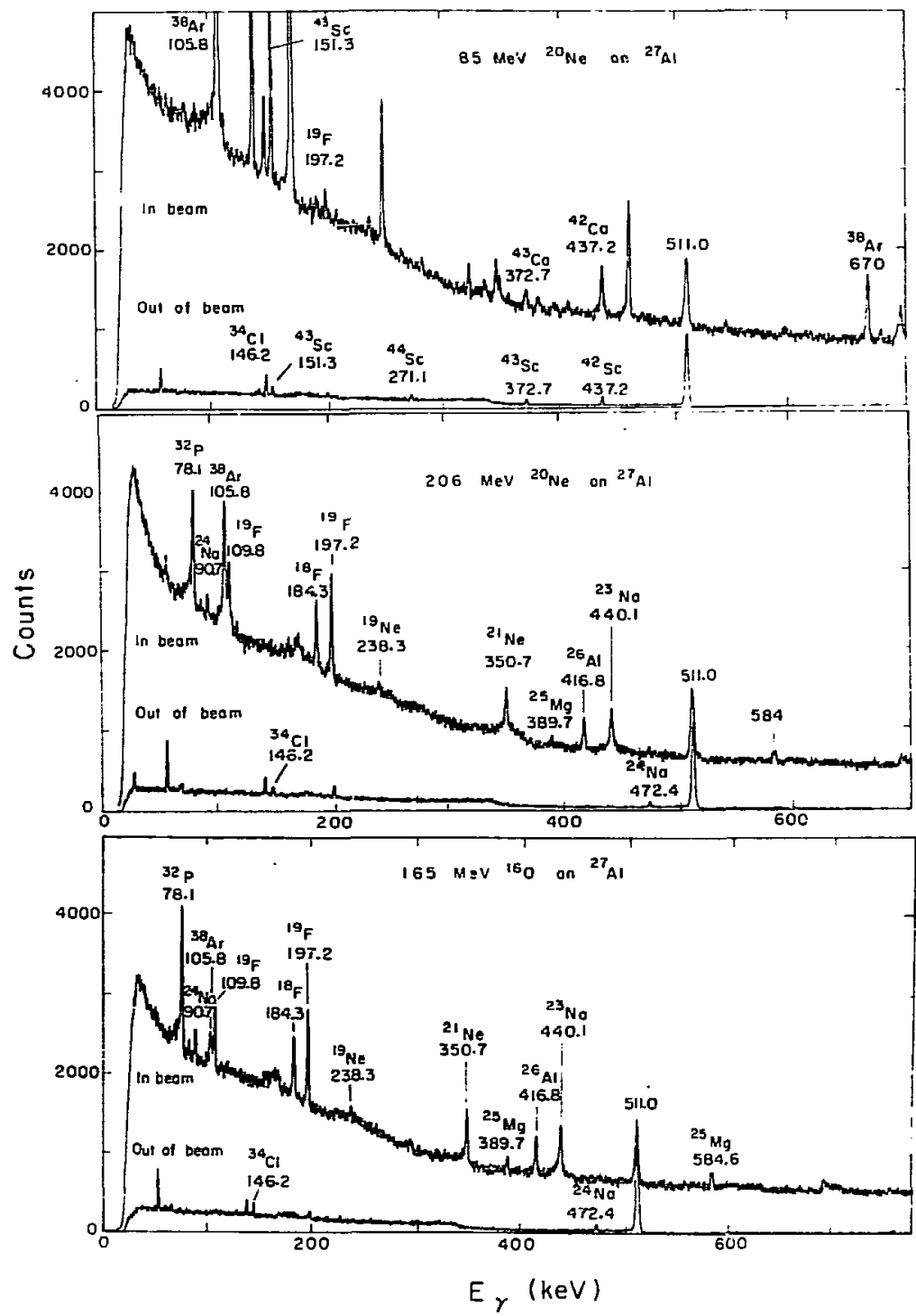

XBI $\gamma 112=\sin$

Fig. 1-3 
Reaction products

in ${ }^{20} \mathrm{Ne}$ on ${ }^{27} \mathrm{Al}$
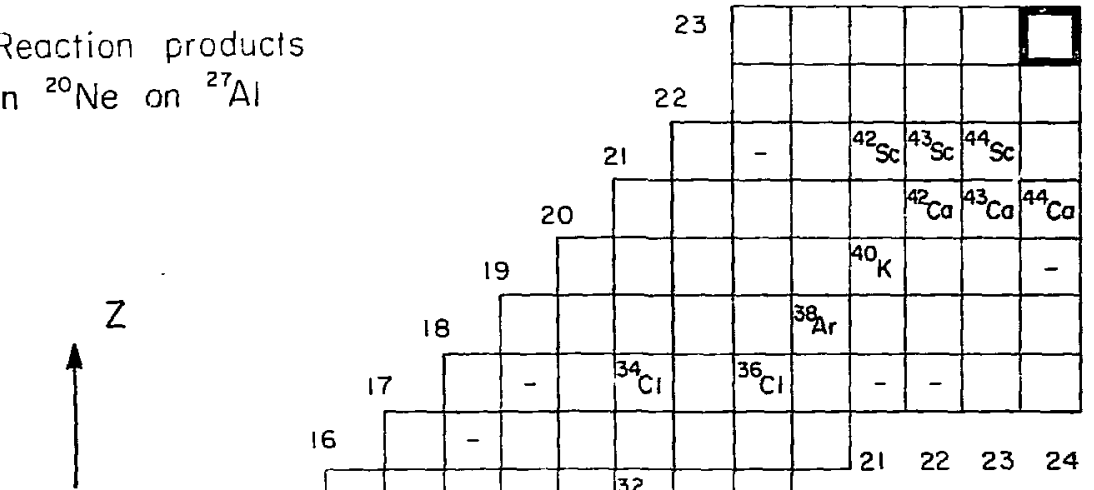

Z

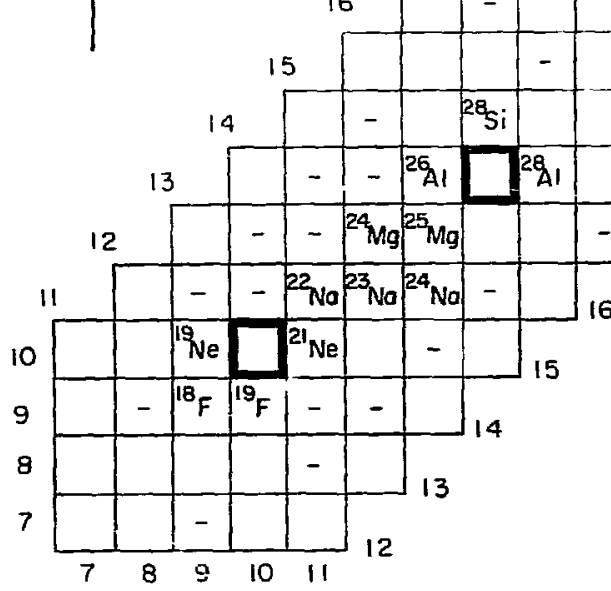

$\begin{array}{lll}22 & 23 & 24\end{array}$

$x 827110-+532$

Fig. 1-4 


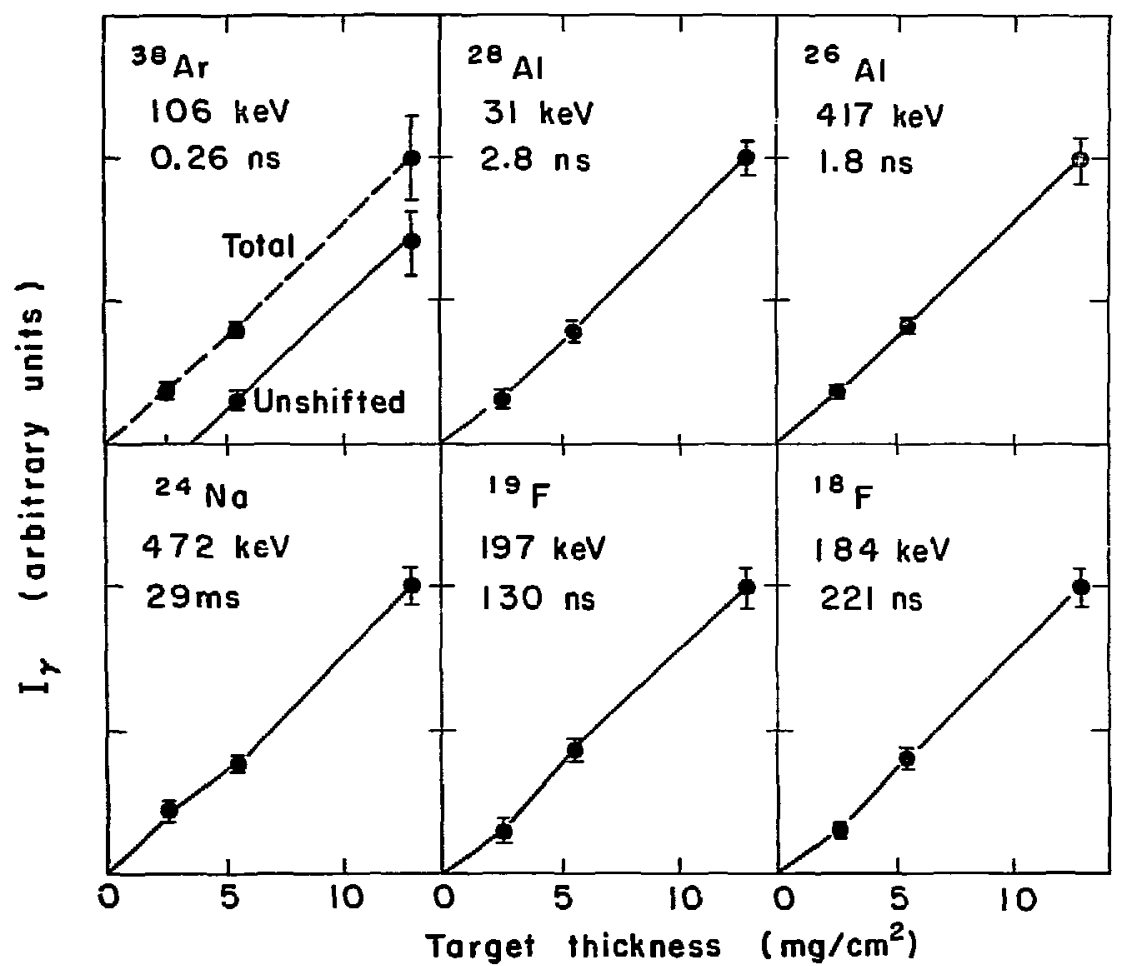

$X B L 7 \| 12-4875$

Fig. 1-5 


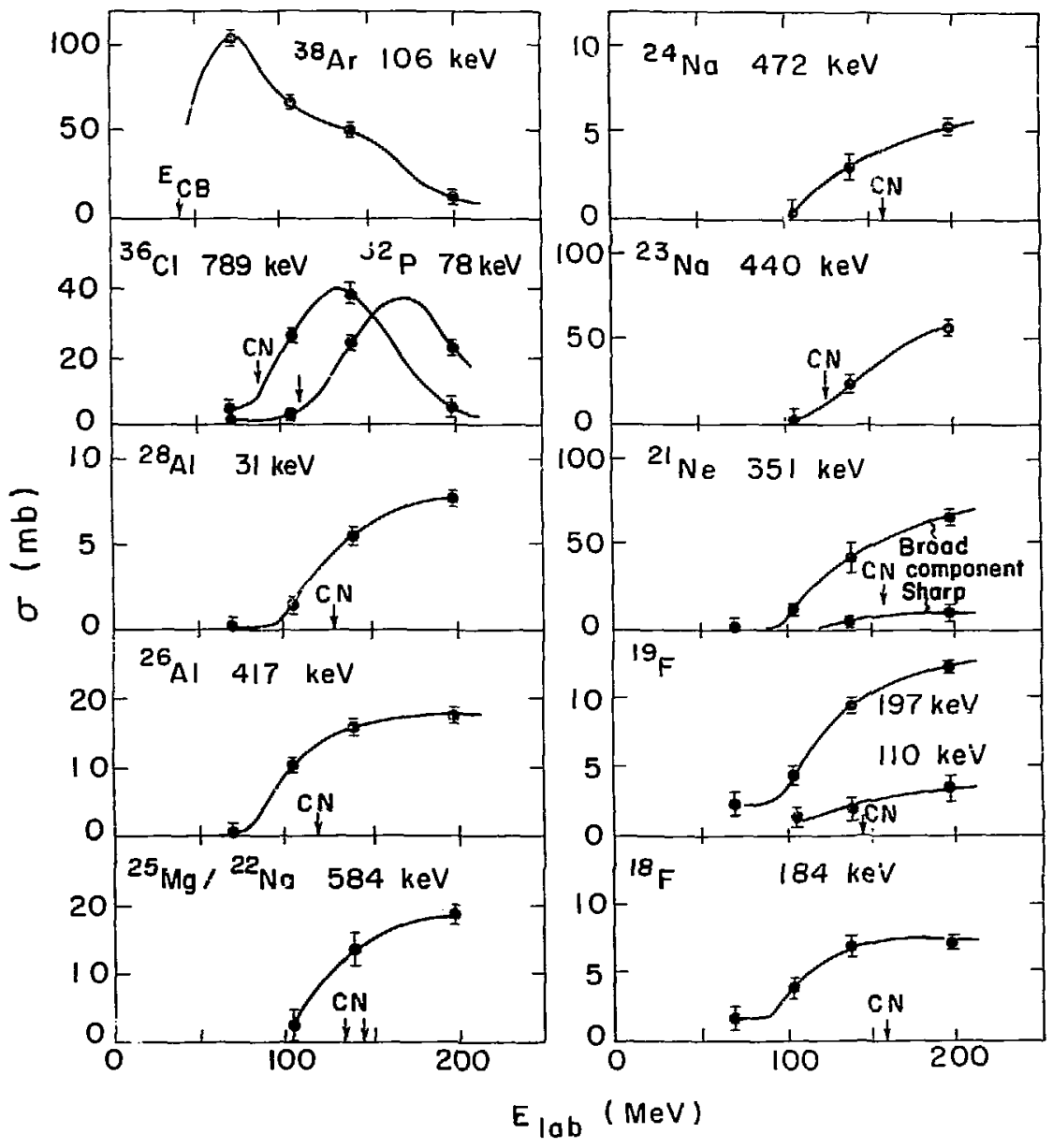

xBL7I12-4876

Fig. 1-6 


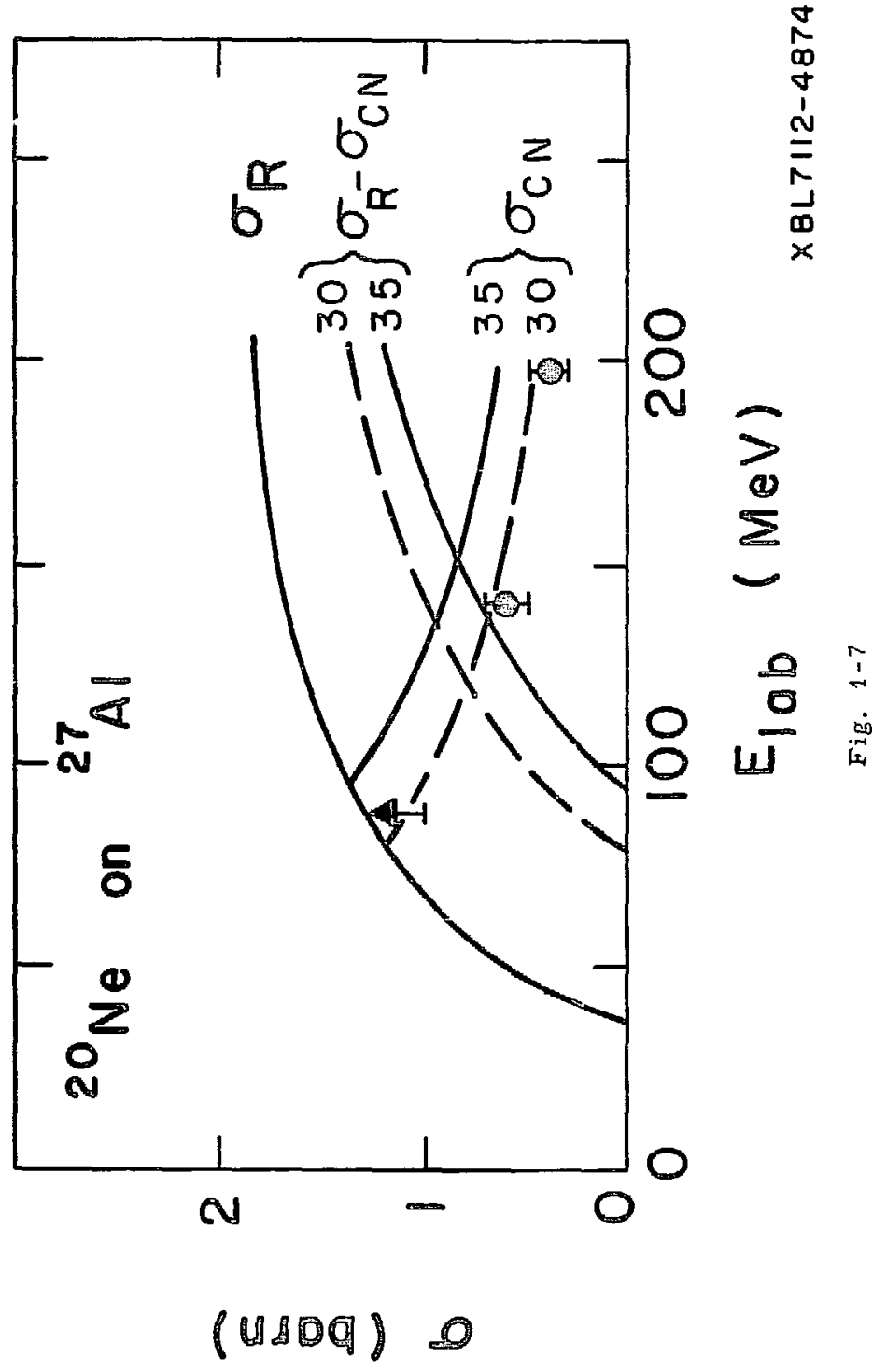




\section{Part II. A New Coupling Scheme and Some Examples}

This morning I want to talk to you about a new coupling scheme for odd-mass nuclei, but I shall start with a little history. About ten years ago, Ray Sheline suggested that there might be a new region of deformation for nuclei with both the number of neutrons and protons in the range of 50-82. When nuclei are well away from closed shells, there are enough particles to polarize and thus to deform the core, and this happens in the rare-earth region, $50 \leqslant \mathrm{z} \leqslant 82,82 \leqslant \mathrm{~N} \leqslant 126$, and in the actinides, $82 \leqslant z, 126 \leqslant \mathrm{~N}$. It might also happen in the new region with both neutrons and protons between 50 and 82. It does indeed seem that there is a tendency towards deformation in this region, as is shown in Fig. 2-1. Here, I have plotted for the Ce nuclei the ratio of the energy of the $4^{+}$state to the $2^{+}$state (which for a rigid rotor would be 3.33 ) and of the $6^{+}$state to the $2^{+}$state (which for a rigid rotor would be 7). The singly-magic nucleus, ${ }^{140} \mathrm{Ce}$, is in the middle of the figure, and you see the ratio is very, very far from 3.3 to 1 . But at the wings, as you move away from the magic number of neutrons, either going to fewer, or to more, than 82 neutrons, these ratios do approach those of the rigid rotor. The lightest nucleus, ${ }^{124} \mathrm{Ce}$, and the heaviest, ${ }^{156} \mathrm{Ce}$, do show approximately the right ratios. So, there is an indication of moving towaras deformation in both of these cases. The neutron-deficient nuclei were studied by us in heavy-ion reactions; the neutron-excess ones were studied in the spontaneous fission of ${ }^{252}$ Cf by stan Thompson's group at Berkeley.

I am going to be interested, primarily, in the neutron-deficient region. If these nuclei are deformed, in what way are they deformed? Are they prolate, are they oblate, are they triaxial? How do you anstrer that question? It turns 
out that it is not easy. You can easily look at the energy levels or the fact that very large B(E2) values are involved and think that you are going towards deformation, but it is very hard to determine experimentally the nature of that deformation. With stable nuclei, you could measure the static quadrupole moment by nuclear reorientation. But, these are not stable nuclei, so how do you find out what is the nature of the deformation? A suggestion has been made that you could tell if you looked at the odd-mass nuclei. In this region - the light cesiums and lanthanums - we are dealing with $h_{11 / 2}$ protans. If you look at a Nilsson diagram for the segment including the $h_{11 / 2}$ proton, you see that on the prolate side the $h_{11 / 2}$ subshell fans out with the $\Omega=1 / 2$ level lowest, and then the $\Omega=3 / 2,5 / 2,7 / 2,9 / 2$, and $11 / 2$ levels in order. On the ablate side the order is reversed, with the $\Omega=11 / 2$ level lowest and $\Omega=1 / 2$ highest. Omega is the component of the particle angular momentum along the nuclear symetry axi 3 , and $\Omega=1 / 2$ means that the $j$ or the particle is almost perpendicular to the symmetry axis, while $\Omega=11 / 2$ has the angular momentum almost parallel to the symetry axis. The point of all this is that it was thought that if you looked at an odd-mass nucleus and identified a low-lying $11 / 2^{-}$spin state or a $1 / 2$ spin state, that would

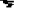
tell you whether the nucleus was oblate or prolate. And so, a lot of work was done looking in the barium and lanthanum and cesium nuclei to try to observe such states. In fact, isomers of spin 11/2 were found in almost all of these odd-mass nuclei. And so the hypothesis was made that these nuclei were oblate, because the only easy way to account for low-lying $11 / 2$ isomers in these nuclei would be if they were on the oblate side. 
Buc the story is not that simple. First of all, if the nuclei were prolate and reasonably deformed, the low-1ying $\Omega=1 / 2$ band would not be regular at all. For such a band, one must consider the effect of the decoupling parameter, which can change the level order in the band if strongly enough deformed. There may not be a regular sequence $1 / 2,3 / 2,5 / 2,7 / 2, \cdot$. , but rather the disturbed order of the strong-coupling limit; the lowest state would be a degenerate pair of levels, of spin $3 / 2$ and $7 / 2$, and the next level would be the 11/2. Thus, you can get a $11 / 2$ state lying low in either the oblate or prolate case. So that is not the conclusive test that it might be. We got interested in this problem and thought that perhaps one way to shed some light on it would be to study, not the lowest-lying level, but the band that is built on that lowest-lying level. The reason for this is given in Fig. 2-2. If the lowest-lying level is a $\mathrm{K}=11 / 2$ level, then there will be a band built on it which is just a normal rotational band. It will have spins $11 / 2,13 / 2,15 / 2,17 / 2,19 / 2, \cdots$ - By using a heavy-ion reaction and the techniques of in-beam spectroscopy, a large amount of angular momentum is given to the product nucleus, and you see a gamma-ray cascade in-beam which will correspond to the left-hand side of the figure. There will be E2 crossover transitions, and Ml-E2 mixtures which will go between states of spin differing by 1. So there will be a rather complicated gamma-ray spectrum, and the level order will be 11/2,13/2,15/2,17/2, . - For the other case on the prolate side, there is, in the strong-coupling limit, a distorted $\mathrm{K}=1 / 2$ band, with the level order as shown in Fig: 2-2. But the reaction makes the nucleus at high spin, so the only states observed will be the hign-spin states at any particular energy; the low-spin 
states are by-passed. The gamma-ray cascade will pass just through the $19 / 2$ state, $15 / 2$ state, and the $11 / 2$ state. There will only be stretched E2 transitions; no Ml-E2 cascades, because the decay only goes between the states of high spin, which differ by two units. So the spectrum would look different. And more importantly, the transition energies are quite different. If you look, foz example, at the spacing between the $11 / 2$ and $15 / 2$ levels, you will see that the energy difference on the oblate side $(K=11 / 2$ band) is more than twice as large as that on the prolate side ( $\mathrm{K}=1 / 2$ band). These are calculated for the same moment-of-inertia. So, if we can identify the $15 / 2 \rightarrow 11 / 2$ transition, we will see a characteristic spacung. For comparison, and because it will be important for what comes later, the energy levels of the even-even core, which go $0^{+}, 2^{+}, 4^{+}, 6^{+}$, - - are also shown in the middle of the figure, calculated with the same moment-of-inertia. When made in a heavy-ion reaction, this gamma-ray cascade will be a series of stretched E2 transitions. Notice that the spacing of the first excited state $(2+0)$ is small. It is $6 \frac{\hbar^{2}}{2 \mathscr{\zeta}}$, and this is about two and one-half times smaller than the $15 / 2+11 / 2$ spacing of the $K=1 / 2$ band and about 4 or 5 times smaller than the $15 / 2+11 / 2$ spacing of the $\mathrm{K}=1: / 2$ band.

So, we* decided to look at the odd-mass lanthanum nuclei in-beam, and to look, in particular, at the band built on the $11 / 2^{-}$state. There are several such isomers known in the heavier lanthanums, and we studied the lanthanums from mass 125 to 137 . In every case, we found an $11 / 2^{-}$isomer, and in the in-beam spectrum we saw a number of strong transitions; one, two, or three of these strong transitions could be identified as forming a stretched E2 cascade feeding the $11 / 2^{-}$isomer. There are also occasionally strong transitions

We are Eugo Maier, of the Hahn-Meitner Institute in Berlin, Kozi Nakai, of the University of Tokyo, Jack Leigh, of the Australian National University, Frank stephens, and myself. 
which were not in that cascade, but they were the ones de-exciting the isomer to ground. In most cases, we worked out how the isomers decayed to ground. That did not always prove that the spin of the isomer was $11 / 2$; the proof that we are looking at spin $11 / 2$ isomers came from $(\alpha, t)$ experiments done in Copenhagen by $\mathrm{K}$. Nakai and $\mathrm{P}$. Kleinheinz, where they bombarded barium with alphas and observed the states in the corresponding lanthanum nuclei. In the heavier nuclei, $11 / 2^{-}$isomers are known, and they found these were strongly excited in the pick-up reaction. For the lighter nuclei, they observed a very similar pattern, so they could claim that these were the $11 / 2$ states in the lighter nuclei. For the two lightest lanthanums, 125 and 127, we do not have proof that they are $11 / 2^{-}$isomers, but we think by analogy that they must be.

I don't want to take up the experimental details, but we did the normal things involved in in-beam spectroscopic studies. We took singles spectra and coincidence spectra, we did angular distributions, and in some cases, we measured the conversion-electron spectra. We took spectra, both in in-beam and between the beam bursts. The resulting decay schemes are in Fig. 2-3. Each odd-mass lanthanum (I left out lanthanum 135 and 137 ) is plotted next to the barium nucleus with one less proton; with the $11 / 2^{-}$state of the lanthanum at the same level as the $0^{+}$ ground state of the barium. I remind you, that these $11 / 2^{-}$levels are not the ground states; they are isomeric states, lying above ground, but we are not interested in that. We are interested in the cascade built on the $11 / 21$ evel. And the striking feature of this figure is that every one of the lanthanums has the same spacing as the neighboring barium. That is, if you take one proton away from the lanthanum, you have the corresponding barium, and it has ground-band -spacings which are identical, essentially, to the lanthanum. (This 
is also true for ${ }^{135,13 ?} \mathrm{La}$; however, in these cases only one higher state is seen and it lies rather high.) This was completely unexpected. A few moments ago I took some time to point out that what we expected was an approximation to either the $K=1 / 2$ or $K=11 / 2$ limiting case, where the $15 / 2$ to $11 / 2$ spacing would be either 2 or 5 times the $2^{+}+0^{+}$spacing of the corresponding even-even core nucleus. And we do not see that at all. The striking feature that we have to explain is, why in this string of odd-mass lanthanums, the spacing of the $11 / 2$ band is the same as the spacing of the ground-state band in the even-even core.

One way you might explain it is by weak coupling. If we weakly couple an $11 / 2$ proton to the even-even core, we get for zero rotation of the core a state which is still 11/2. That is the isomer, the head of the band. If we couple the $11 / 2^{-}$particle to spin $2^{+}$of the core, we get five states with spins ranging from $7 / 2^{-}$to $15 / 2^{-}$. Coupling $11 / 2^{-}$to the $4^{+}$core state gives a group of degenerate states whose spins range from $3 / 2^{-}$to $19 / 2^{-}$. Now, if the splitting of these levels - the breaking of the degeneracy of these levels - is very small, then you have the weak-coupling model, and clearly the spacing between the groups of states is still the same as the core spacing. If you pick out the states in each multiplet of highest spin, you will get the 19/2, 15/2, 1l/2 spacing observed in the odd-mass La. But, we do not think that is the answer for a number of reasons. One is experimental. If weak coupling were occurring, we should see several transitions between the members of the multiplets, as they are not very different in energy. For example, if you make the 19/2 staie, you are also going to make the $17 / 2$ state. You may not make as much of the $17 / 2$ as the 19/2, but you will surely make an observable amount. And so, you should see 
the $17 / 2$ decaying to the $15 / 2$ and $13 / 2$ states. The latter transition would not have exactly the same energy as the $19 / 2+15 / 2$. OI, if it had exactly the same energy in one cass, it would not have the same energy in all of these cases. And in all of thest: cases, we see only a single transition between each multiplet. So, we do not think this is weak coupling. And you will see in a moment that we are working in a region where the splitting of the multiplet levels is greater than the spacing of the core states, exactly the opposite of the weak-coupling requirement.

We obtained these results about $2 \frac{1}{2}$ years ago, and at that time we had no explanation. Tilen, about two years ago, Frank Stephens went to Munich on leave. During his year there, he worked with a student on a theoretical problem involving the Coriolis coupling of two high-j particles in an even-even nucleus in order to explain (and I don't want to get into this) the backbending phenomenon seen in some nuclei for high-spin states of the ground band. But, when he came back to Berkeley a little over a year ago, we talked about this explanation, and it occurxed to us that if it had any validity for the even-even case, it should be still simpler for a single odd-particle coupled to a core, and so we ought to investigate that situation. The idea is really quite simple, and I want to give you the physical idea first. Iet us start, not with the weak-coupling scheme, but with the strong-coupling model of Bohr and Mottelson. We take an even-even deformed core and add a high-j particie. The single particle rotates around the nuclear symetry axis very rapidly, faster than the core rotates (at right angles to the symmetry axis). Thus, in the limit, the projection of the particle angular momentum $j$ on the symmetry axis is a constant of the motion (usually expressed as a value of $\Omega$ ). This is the rigid-rotor model of Boh. 
and Mottelson. The energy levels of this nucleus will be given by the simple rigid-rotor formula, $E(I)=\frac{h^{2}}{2 J} I(I+1)$ where $\vec{R}$ and $\vec{j}$ couple to $\vec{I}$. What this is saying is that when the nucleus is very deformed (has a large value of (f) in this limit, the most economical way for the nucleus to carry angular momentum is to rotate as a whole. Now, what happens if we decrease the deformation of this nucleus, of the core?

The moment-of-inertia is proportional to $\beta^{2}$ in the simple hydrodynamical model, so if ô becomes smaller, the constant $\frac{1}{5}$ becomes larger, and the cost in energy of rotiating the nucleus goes up. At some point as you decrease $B$, it may become more economical, energy-wise, for the high-j odd-particle, say an $h_{11 / 2}$ proton, to align itself along the rotational axis rather than to give another 5 units of rotation to the core. The question then is, how much energy does it cost to so align the particle? This amount of energy is related to the spread in energy for different values of $\Omega$ in the Nilsson diagram. But since the energy splitting of a j-shell in the Nilsson diagram is roughly proportional to $B$, the energy cost is smaller as $\beta$ decreases (the levels becare degenerate at $\beta=0$ ). That is, as the deformation decreases, the coupling of the particle to the symmetry axis is weakened, and it becomes easier to change the particle orientation at the same time as it becomes more difficult to rotate the core. For a high-j particle, its alignment along the rotation axis may free considerable core rotational energy. When the particle is optimally aligned along the rotating axis, the energy differences between different spin states become just the energy diffe:cences of the core states, and the states differ by $2 h$, as do the core states. The aligned particle contributes the same spin and energy to each state. 
What values of $\Omega$ does this particular configuration of $j$ involve? It is obvious that $\Omega=1 / 2$ is a very important component, as it has the smallest projection along the symmetry axis, and so the largest along the rotation axis. But a pure $\Omega=1 / 2$ state has as much probability of being oriented anti-paralleI to the rotation axis as parallel, and so a mixture of $\Omega$ states, a wave packet, must be created. This wave packet has $\Omega=1 / 2$ as its largest component, but also involves $\Omega=3 / 2,5 / 2,7 / 2 \cdot$. - j components with the proper phases to produce a spatial orientation parallel to the rotation axis. Actually, this will lead to a new coupling scheme in which the projection of $j$ that is considered is not that along the symmetry axis, $\Omega$, but that along the rotation axis, $\alpha$.

I have a model here to demonstrate the scheme. A little gyroscope is built into a rotating irame. The little gyroscope represents the particle spinning on its own axis. The frame is the prolate nuclear core. The "core" and the "particle" can rotate independently. I couple this particle strongly to the symmetry axis and let the core rotate. That is Bohr and Mottelson's strong-coupling model. Now, if I weaken the coupling of this particle to the symmetry axis, and now rotate the core, what happens? The particle angular momentum axis aligns itself with the rotational axis of the core. The Coriolis interation plays a major role in causing this alignment, and when the rotating particle is aligned, the coriolis force on it is zero. But, if it moves away from the axis, the force pushes it back into alignment. I show you essentially the same picture in Fig. 2-4. The big wheel is the core, and the smaller wheel represents the particle rotating at right angles to the big one. If the small wheel is 
strongly coupled, that is fixed, to the bigger one, it has to rotate with it; that is the strong-coupling limit. But, if the coupling to the big wheel could be weakened, the little wheel will align itself with the axis of rotation of the table, and the latter can rotate more slowly for the same total angular momenturi. So, that is the physical picture of our coupling scheme. To go any further, we will have to do some calculations. We shall take a very simple model, a particle in a single-j shell, the $h_{11 / 2}$ shell, coupled to an even-even core. The core is ceformed and axially symmetric. The Hamiltonian is composed of two parts, the rotation of the core and the interaction of the particle with the deformed potential of the core.

$$
\mathrm{H}=\mathrm{H}_{\text {intr }}+\frac{\hbar^{2}}{2 \mathrm{z}} \overrightarrow{\mathrm{R}}^{2}
$$

If there is no direct particle-core interaction, then the energy depends only on the rotation of the core. This is the weak-coupling limit. Alternatively, we may start with the strong-coupling limit, and then work back into the intermediate region. To do that, we merely replace $\vec{R}$ by $\vec{I}-\vec{j}$ and expand eq. (1).

$$
\left.\mathrm{E}=\mathrm{H}_{\text {intI }}+\frac{\hbar^{2}}{28}\left[I(I+1)-\Omega^{2}\right] \frac{\hbar^{2}}{2 \xi}\left(\dot{j}^{2}\right)-\Omega^{2}\right)+H_{C}
$$

where the coriolis operator $H_{C}$ can be written as

$$
\begin{aligned}
\mathrm{H}_{c} & =-2 \cdot \frac{\hbar^{2}}{2 J^{2}}\left[\vec{I} \cdot \vec{j}-\Omega^{2}\right] \\
& =-\frac{\hbar^{2}}{2 \delta}\left[I_{+} j_{-}-I_{-} j_{+}\right]
\end{aligned}
$$


We don't change the Hamiltonian; we are essentially changing the basis functions. We are going to use the strong-coupling wave functions because we know what they are. We can diagonalize eq. (2) for a given spin and see what the calculation will give over the whole region of coupling. In the strongcoupling limit where the nucleus is very deformed, the Coriolis term becomes very small because $\frac{1}{\vec{F}}$ becomes small and because the spacings between the states of different omega become very large. The second and third terms in the Hamiltonian are diagonal, and the only changeable term is $I(I+1)$. So we have the formula for the rigid rotor in the strong-coupling limit. To make calculations, we must specify the nature of $\mathrm{H}_{\text {intr }}$. The logical potential to use is the Nilsson one, but this is complicated, so that we will use a linear approximation to it instead,

$$
E(\Omega)=E_{0}(n \ell j)+\frac{206}{A / 3} \beta \frac{3 \Omega^{2}-j(j+1)}{4 j(j+1)} \text { (MeV) }
$$

This is a very good approximation, even to $\beta \approx 0.3$, for the $h_{11 / 2}$ proton, because this particular subshell is a unique-parity subshell ir the Nilsson diagram. It cannot mix with other states in its shell, only with those in the shell above, and so does follow a linear relationsthip. We also approximate the effects of pairing correlations by the usual method of working with quasi-particle energies rather than single-particle energies.

$$
E(\Omega)=\sqrt{(\varepsilon(\Omega)-\lambda)^{2}+\Delta^{2}}-\Delta
$$

where $\lambda$ is the Fermi surface and $\Delta$ is the gag, irameter. This is not important for the solution to the problem; it changes the answers a little, but not in an important way. 
We now have a Hamiltonian to diagonalize which contains four parameters for a given I. They are $\frac{\hbar^{2}}{2 \xi}, \beta, \lambda, \Delta$. These are too many. The gap parameter, $\Delta$, we can fix at $0.8 \mathrm{MeV}$, since the results are not sensitive to its value. We also know the relationship Grodzins pointed out many years ago becween the lifetime of the first $2^{+}$state in an even-even nucleus (anywhere in the periodic table) and the energy of that $2^{+}$state. The lifetime is inversely proportional to the fourth power of the energy. What that really means is a relationship between the moment-of-inertia and $B$, because you can relate the energy to the moment-of-inertia, and you can relate the lifetime to the $B(E 2)$ and the $B(E 2)$ to $\beta^{2}$. So we have a relationship between $\beta$ and $\frac{h^{2}}{2 \mathcal{J}}$, and we can eliminate $\frac{h^{2}}{2 \xi^{\circ}}$. We end up with two parameters, $\beta$ and $\lambda$; $B$ is the measure of the deformation, and $\lambda$ is the Fermi energy. We shall take $\lambda$ to be the energy of the odd (last) particle we are dealing with on the Nilsson diagram. When we vary $\lambda$, we shall be varying the valur of $\Omega$ for this last particle. Now with two parameters, we can fix one of them arbitrarily, and then :iagonalize the matrix and see how the energy levels change as we vary the other parameter.

Figure 2-5 $\Xi^{\prime}$ is the energy levels as a function of $\beta$, with $\lambda$ fixed on the $\Omega=1 / 2$ state. $B$ goes from 0 to +0.3 ; this figure unfortunately only indicates the prolate side. I shall come back to the oblate side below. The ordinate is the energy of the state of spin I minus the energy of the lowest-lying state of spin $11 / 2$ divided by the energy of the $2^{+}$core state. 


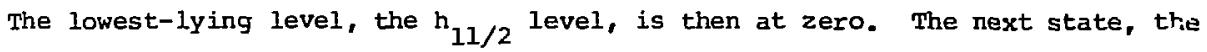
multiplet built on the core state of spin 2, appears for $\beta=0$ at unity on the vertical axis, and as $\beta$ increases, it breaks up into five levels with spir:j fram $7 / 2$ to $15 / 2$. When $B$ is equal to 0.2 , (approximately the value of $B$ for the odd-mass Lu isotopes described earlier) the spread of these levels is far greater than the spacing of the core, and that is why I said earlier a weakcoupling model is not applicable to the La situation. A much smaller value of $\beta$ is necessary to yield the weak-coupling limit $(\beta<0.08)$. However, for small $\beta$, this calculation gives the right order of the levels, if only quadrupole-quadrupole interactions are considered in that limit. And in the weak-coupling limit, the order of these levels is different than that shown in Fig. 2-5 for $\beta>0.1$. But, the striking feature of Fig. 2-5 is that the state of highest spin in each multiplet is behaving in a very special way; thus, all stay parallel with the $11 / 2$ state. That is, the lowest $15 / 2,19 / 223 / 2, \cdots \cdot \because$ states are staying parallel to the $11 / 2$ state over the range of $B$ from 0.0 to 0.3 ;

they keep the same spacings. It is very important to remind you that in these calculations we have fixed $\lambda$, the Fermi surface, on frecisely the $\Omega=1 / 2$ state. That is, the oad particle is precisely on the $\Omega=1 / 2$ orbit of the $h_{11 / 2}$ subshell. The Ia nuclei with which we staried this dissenssion, do have $\lambda$ approximately on the $\Omega=1 / 2$ level (just below) and their values of $\beta$, estimated frcm the corresponäing even-even Ba cores, range from 0.15 to 0.25 . For that region in Eig. 5, the spacing of the highest-spin members of each multiplet is closely the spacing of the core itself. So in fact, we calculate exactly what we have observed experimentally, and although there vas no good reason, a priori, why it should work, we can say that the remajkable agreement indicates the essential validity of the model. 
However, there is an obvious drawback to this model. It is based on an axially symmetric nucleus whose core has the energy spacings of a pure rotor,

$$
E(I)=A I(I+1)
$$

That feature is not true for any of these nuclei, as the Ba cores are not good rotors, but rather poor ones. Thus, we must determine whether or not it is essential to this picture that the core be a good rotor.

The customary thing to do when energies cannot be fit by eq, (2-6), is to add another term,

$$
E(I)=A I(I+I)+B I^{2}(I+I)^{2}
$$

where $B$ would be negative, and is supposed to account in first order for rotation-vibration interaction. The fits are better. If still not good enough, a third term in $\mathrm{CI}^{3}(I+1)^{3}$ is added. This is not a good expansion for the energy, because you sometimes need only one less term then you have energies to fit. But it works for not too high spins. So we fitted the levels of the Ba core by a three-term expansion and obtained vaiues of $A, B$, and $c$. Then we used these values in an expanded Hamiltonian where we added a $\mathrm{BR}^{4}$ and $a \mathrm{CR}^{6}$ term to the $\mathrm{AR}^{2}$ of $\mathrm{eq}$. (2-I). Since with the $\mathrm{B}$ and $\mathrm{C}$ terms we can fit the $\mathrm{Ba}$ core energies, we can see if we still obtain the right behavior for the $15 / 2,19 / 2,27 / 2$ - * levels in this new calculation. In this more complicated Hamiltonian, we again replace $\vec{R}$ by $\vec{I}-\vec{j}$ and obtain an enormous number of terms. It is a very complicated coriolis calculation to do by hand, but the computer can handle it. For $\beta=0.2$, we 
get the dots in Fig. 2-5. So, in fact, we do not change the results much, and the highest-spin members of the multiplet stay at about the same energy as the Ba core states, even though these are not rotational spacings.

These calculations were made holding $\lambda$ elong the $\Omega=1 / 2$ state, and varying $\beta$. Now let us hold $\beta$ constant at $\beta=0.25$ and vary $\lambda$. (see rig. 2-6.) The energy of $\lambda$ above the $\Omega=1 / 2$ state appears on the bottom and the corresponding $\Omega$-state appears at the top of the figure. The previous figure was for $\Omega=1 / 2$, and that yielded the $11 / 2,15 / 2$, and $19 / 2$ states at the core separation. For $\lambda$ on the $3 / 2$ state, you still have the same order and the same spacings, and they remain just about the same for $\lambda$ on the $5 / 2$ state. But, as $\lambda$ rises to the $\Omega=7 / 2$ state, the order is clearly changing. For $\lambda$ on the $\Omega=9 / 2$ state, a good rotational spectrum for a $9 / 2$ rotational band appears, and when $\lambda$ is on the $\Omega=11 / 2$ state, just a normal $1 i / 2$ rotational band occurs. The order becomes that of a good rigid rotor in the strong-coupling limit. Now, why is the coupling changing? In the new coupling scheme we have $j$ aligned along $k$ with a constant projection $\alpha$ - (See Fig. 2-7.) The rotation-aligned wave function needs a lot of the $\Omega=1 / 2$ camponent. That is the majox component in the wave function. So obviously, that scheme works best if the odd particle is in or near the $\Omega=1 / 2$ state. But if the particle is in the $\Omega=11 / 2$ state, it may cost many MeV to use the $\Omega=1 / 2$ component. So it becomes very hard to get rotatior-alignment if the particles are filled up to the $11 / 2$ state. In this perticular case of $\beta=0.25$ the new scheme holds From $\lambda$ at or below the $\Omega=1 / 2$ state to $\lambda$ up to the $\Omega=5 / 2$ state. If $B$ is made smaller, then this holds to still larger values of $\lambda$, as the levels come closer together. If $\beta$ is linger, then it works only to smaller values of $\lambda$, 
as the levels become farther apart. The values of both $\beta$ and $\lambda$ are important, and this new scheme should work when $B$ is small and whe, $\lambda$ is near the $\Omega=1 / 2$ level.

For the La isotopes, we are at $\Omega=1 / 2$, in fact, the Fermi surface is below the $\Omega=1 / 2$ level. The value of $\beta$ is between 0.15 and 0.25 , and so the new coupling scheme should occur. The result should be a band of levels with the Ba-core spacings, but with spins $11 / 2 \mathrm{~h}$ higher than the core states, just as was observed experimentally. But in addition, from our discussion we can see that this can only happen when $\lambda$ is near the $\Omega=1 / 2$, and not the $\Omega=11 / 2$, state, and this tells us that the La nuclei must be prolate, and not oblate as had been thought.

So we can explain these data with this model. But if it is any good, it should be possible to predict things. First of all, this calculation gives the wave function, so you can predict transition moments, static moments, etc. I don't want to go into these. But more importantly, if this idea is right, there is nothing special about the La isotopes. The same kind of behavior should occur anytime the odd-particle is in a high $j$ orbital with $B$ not too large and with $\lambda$ near the $\Omega=1 / 2$ orbital. For example, let's go to a particle of still larger $j$, the $i_{13 / 2}$ neutron. The coriolis interaction should be stronger, and the new coupling scheme should work fine as long as $\beta$ is not too large and as long as the odd neutron is near $\Omega=1 / 2$. Such nuclei are in the rare-earth region. As examples, the odd-mass exbiums have been studied quite a bit. The group in storkholm studied ${ }^{165} \mathrm{Er},{ }^{163} \mathrm{Er}$, and ${ }^{161_{\mathrm{Er}} \text {, and }}$ we studied ${ }^{161} \mathrm{Er},{ }^{159} \mathrm{Er}$, and ${ }^{157} \mathrm{Er}$. The decay schemes are shown in Fig. 2-8. The situation is somewhat more complicated than in the La isotopes, because now as the number of neutrons decreases, two things happen simultaneously. In going 
to lighter Er isotopes, $\lambda$, the Fermi surface, changes from $\Omega=5 / 2$ to $\Omega=1 / 2$, and at the same time, $\beta$ changes from $\sim 0.35$ to $\sim 0.15$. Both $\beta$ and $\lambda$ are decreasing, and that is forcunate, because both of these changes are such as to favor the rotation-alignment coupling scheme. In Fig. 2-8 you will notice that for ${ }^{165} \mathrm{Er}$, the bottom level is a $5 / 2^{+}$state. The band is an almost normal rotational scheme, but with a distortion in the spacings becoming more evident at the higher spins. This is the influence of the coriolis interaction in mixing in the $\Omega=1 / 2$ band from the same $i_{13 / 2}{ }^{+} j$-subshell. That is, this is the effect of the decoupling parameter in the $\Omega=I / 2$ band, and can be calculated. The stockholm group has done this, and obtained good agreement between experiment and theory. As we go to lighter Er's, the Coriolis interaction becomes stronger, the alternation in the levels becomes much more marked, and so by ${ }^{159}$ Er, only the $j+2 n(n=0,1,2, . \cdot$ ) levels are observad. The other states exist, but they have moved up in energy, while the observed levels approach the core spacing. To show you that these latter levels do approach the core spacing, the next figure, Fig. 2-9 compares the odd-mess Ex's with their even-even neighbors. The $13 / 2^{+}$levels of the odd-mass nuclei are plotted at the same level as the $0^{+}$states of the doubly-even core nuclei. Here ${ }^{166}$ Er and ${ }^{165}$ Er are fairly good rotors, and reasonably near the strong-coupling limit. The $17 / 2$ to $13 / 2$ spacing in the odd nucleus is larger than the $2^{+}$to $0^{+}$spacing in the even one, more than 2 times as big. But in the strong-coupling limit it must become almost 5 times larger, so it should continue to rise in the stizl heavier odd-Er isotopes until it gets above the $4^{+}$state. If we go to lighter nuclei, decreasing $B$ and $\lambda$, then we should come to the new coupling scheme. At ${ }^{157} \mathrm{Er}$, the $17 / 2$ to $13 / 2$ spacing is indeed half-way between the $2^{+}$to $0^{+}$spacing in its neighboring even-even cores, ${ }^{156} \mathrm{Er}$ and ${ }^{158} \mathrm{Er}$. There is a smooth transition in between, and that is 
also true for the higher spin states. We also obtained data on ${ }^{155}$ Er, but at the time we did not understand them, because the transitions looked like those of an even-even nucleus. Now it is quite clear. It does look like an even-even nucleus, because it has precisely the spacing and stretched E2 cascade of an evan-even nucleus.

still other examples can be found around proton number 82 , where the odd particle may be either an $h_{11 / 2}$ or $h_{9 / 2}$ proton, and where we have four possible cases:

1. prolate, strong coupling

2. prolate, decolipled

3. oblate, strong coupling

4. oblate, decoupled

We have examples for all four of these possible situations in the Tl, Re, and Au nuclei.

First, let us consider Fig. 2-10, the Nilsson diagram of this region, with the $h_{11 / 2}$ and the $h_{9 / 2}$ orbitals going from $\beta=-0.3$ to to.3. (Remember that, in our calculation, we use a linear approximation in $\beta$ for the Nilsson potential.) In our discussion, there were two conditions for the realization of the new coupling scheme; 1) moderately small values of $\beta$, and 2) a Fermi surface near the $\Omega=1 / 2$ orbital. In the $\mathrm{La}$ isotopes, we are at the beginning of the $\mathrm{h}_{11 / 2}$ shell, and so on the prolate siae, $\lambda$ is near (below) the level of $\Omega=1 / 2$, and on the oblate side, $\lambda$ is near the level of $\Omega=11 / 2$. Thus, we have a normal coupling scheme for $\beta<0$ and we have the new coupling scheme for $\beta>0$. Since we observed decoupled bands, the Ia nuclei must be prolate. Now consider the nuclei around the 82proton shell. In this region, we are near the $\Omega=1 / 2$ level for $h_{9 / 2}$ protons on the prolate side (this could lead to the new coupling scheme), and on the oblate side we are near the $\Omega=9 / 2$ orbital, which will lead to a normal rotational scheme. On the other hand, or the oblate side we are near the $\Omega=1 / 2$ orbital 
for the $h_{11 / 2}$ proton (therefore, the new coupling scheme may be expected), and on the prolate side we will be near the $\Omega=9 / 2$ or $11 / 2$ orbital for the $h_{11 / 2}$ proton (this should yield a normal coupling scheme). Thus, we might expect all four possible examples.

Let us look first at the case of the $h_{9 / 2}$ proton. We know in the odd-mass Tl nuclei of the existence of a $9 / 2^{-}$isomeric state, which we believe is due to the excitation of a proton across the gap to the $h_{9 / 2}$ - orbital. In Fig. 2-11, we have calculated the spacings of the various levels as a function of $\beta$ with a value of $\lambda$ fixed $2.5 \mathrm{MeV}$ below the energy of the $h_{9 / 2}$ level at $\beta=0$. We estimate a value of $\beta$ for ${ }^{199} \mathrm{Tl}$ from the value of the even-even core, ${ }^{198} \mathrm{Hg}$, as $|\beta|=0.11$, and show the position of these values. Experimentally, we find in ${ }^{199} \mathrm{Tl}$ the spin sequence $9 / 2^{-}, 11 / 2^{-}, 13 / 2^{-}$, and $15 / 2^{-}$, and plot these states as dots on the figure. In comparision with the calculated results, we can see that the order is approximately right on the oblate side for $\beta=-0.11$, but the spacing is not very good, especially for the $15 / 2^{-}$state. But it should be rememberea that the TI nuciei are not good rotors, and yet the calculation was done as if ${ }^{199} \mathrm{Tl}$ is a perfect rotor. Therefore, we will fit the energy levels of ${ }^{198} \mathrm{Hg}$ by the expansion $\mathrm{E}_{I}=\mathrm{AI}(I+1)+\mathrm{BI}^{2}(I+1)^{2}+\mathrm{CI}^{3}(I+1)^{3}$, and then tise these values of $A, B$, and $C$ to add $B R^{4}+C R^{6}$ terms to the Hamiltonian to ba diagonalized. Including these terms, we obtain the modified energy levels show as dished curves in Fig. 2-11, and they do fit to the experimental points rather well. This can perhaps ba better seen in Fig. 2-12, which compares the experimental level scheme for ${ }^{199} \mathrm{Tl}$ with those calculated for the simple Hamiltonian and the ex -ended one. Remember that this is a no-parameter $f i t$, as the values of $\beta$ and of the core spacings are taken from ${ }^{198} \mathrm{Hg}$. Thus, for the $\mathrm{h}_{9 / 2}$ proton on the oblate side, exactly what was expezted was observed, i.e., a rotational scheme: $9 / 2^{-}, 11 / 2^{-}, 13 / 2^{-}$, and $15 / 2^{-}$. 
We show another example illustrating the prolate side. In the case of the isotopes ${ }^{179,177} \mathrm{Re}$, Newton and his colleagues have identified a band based on the $\mathrm{h}_{9 / 2}$ - proton. We take the deformation of these nuciei from the average value of the neighboring even-even core nuclei ( $W$ and Os) to be $|\beta|=0.24$. In these Re isotopes, the $5 / 2^{-}, 9 / 2^{-}, 13 / 2^{-}, 17 / 2^{-}$states of this band are observed experimentally, and the level spacing for ${ }^{179}$ Re is shown zo dots in Fig. 2-11. It is seen that in this more deformed nucleus, the simple Hamiltonian alone gives a good fit (remember with no-parameters), and it is clear that the band is prolate and is close to a decoupled band; the value of $B$ is too large and the $5 / 2$ state has dropped below the $9 / 2^{-}$.

Now look at the result of calculations for the $h_{11 / 2}$ proton shown in Fig. 2-13. The bottom figure has $\lambda$ fixed at $1.0 \mathrm{MeV}$ above the spherical $\mathrm{h}_{11 / 2}$ orbitals (position at $\beta=0$ ). In ${ }^{179,177} \mathrm{Re}$ again, Newton et al. also identified a band based on the $h_{11 / 2}$ proton, and for these cases the Fermi surface is above the $h_{11 / 2}$ subshell, as plotted. Again the position of $|\beta|=0.24$ is indicated, and the level spacing of this $h_{11 / 2}$ band in ${ }^{179}$ Re is shown by dots. Clearly only the prolate side is possible, leading to a strong-coupled $\Omega=9 / 2$ band, and although the fit is not perfect, again this involves no free parameters. The $h_{11 / 2}$ and $h_{9 / 2}$ Re bands are consistent in both requiring a prolate shape, and they furnish examples of both a normal band and a (almost) decoupled band.

The last example I want to show you involves the neutron-deficient gold nuclei, $193,195 \mathrm{Au}$. Since the atomic number of Au is greater than that of Re, the value of $\lambda$ lies a Iittle higher, by 1 Mev, above the $h_{11 / 2}$ orbitals, and the appropriate calculated levels are shown in the upper part of Fig. 2-13. They are 
not very different from the lower part. Again determining the value of $\beta$ for ${ }^{195} \mathrm{Au}$ from the average of its even-even neighbors (Pt, $\mathrm{Hg}$ ) yields $|\beta|=0.12$, and these two deformations are indicated on the figure. clearly the oblate side of the figure is required to fit the experimental points, but the fit is not too good. Again as with TI, this is probably because the value of $\beta$ is sma11, the core is not a good rotor. So the same procedure was followed; the $0,2^{+}, 4^{+}, 6^{+}$levels of the core were fitted by a three-term power series, and the values of $A, B$, and $C$ used in the expanded Hamiltonian shown in Fig. 2-14. Again, there was a marked improvement in the fit. In this nucleus, there is a special feature, From B-decay studies, some of the lower-spin members of the band have been observed, and the $7 / 2,9 / 2$, and $3 / 2$ members also fit the calculations. And clearly those levels that come from the same multiplet, e.g., 7/2, $15 / 2,9 / 2$, show spacings which are large compared to the core spacings; this situation is certainly not weak coupling. The $13 / 2$ level does not fit the calculation. Only time will tell why not. But for such a very simple model, the results so far have been very gratifying, and we know of still more examples sf decoupled bands in the odd-mass mercury isotopes and other nuclei. 


\section{Part II}

Figure Captions

Fig. 2-1. Ratio of the energy of the level of spin $I$ to that of spin 2 for the known ceriun nuclei.

Fig. 2-2. Energy-level diagram for a well-deformed $j=k=11 / 2$ band (left), a well-deformed $j=11 / 2, K=1 / 2$ band (right), and à well-deformed doubly-even nucleus (center); all have the same value for $\hbar^{2} ; 2 \tilde{\delta}$.

Fig. 2-3. Energy levels of the odd-mass lanthanum and the neighboring doubly-even barium nuclei. The $11 / 2^{-}$levels in the lanthanum nuclei are placed at the Fosition of the $0^{+}$ground state in the barium isotopes.

Fig. 2-4. a) The small wheel is constrained to rotate perpendicular to the axis of rotation of the larger wheel. If this colpling is broken, the Coriolis interaction will align the small wheel along the axis of rotation of the larger one, b).

Fig. 2-5. The solution to eq. $(2-2)$ for the $h_{11 / 2}$ orbital for various values of $\beta\left(\frac{A}{130}\right)^{2 / 3}$ (top) with the Fermi surface fixed on the $\Omega=1 / 2$ state. In the limit of large $R$, the level scheme will become a pure $\Omega=1 / 2$ band with a decoupling parameter of -6 . The ordinate j.s the difference in energy from the $11 / 2^{-}$level in units of $\mathrm{E}_{2+}$, the energy of the first excited stata of tr. core. Tr $\equiv$ dots show the effect of adding a $B$ ar.: $C$ tcm, ytere the values of $B$ and $C$ were taken from the fit to the lowest levels in ${ }^{126} \mathrm{Ba}$. Fig, 2-6. The effect of vaiying $\lambda$ is shown for a fixed value of $B=0.25$. At tine top the position of the various $h_{11 / 2}$ component levels is shown. 
Fig. 2-7. Schematic vector diagrams illustrating the deformation-dligned coupling scheme (above) and the rotat som.ligned coupling scheme (below). Fig. 2-8. Decay schemes of the odd-mass erbium isctopes.

Fig. 2-9. Systematics of the $13 / 2^{+}$band in the odd-mass erbium nuclei and of the ground-state band in the doubly-even isotopes.

Fig. 2-10. Part of the Nilsson diagram around the 82-proton shell. The ground states of $\mathrm{Al}$ and $\mathrm{Tl}$ - $\mathrm{e}$ in the $s_{1 / 2}$ and $\mathrm{d}_{3 / 2}$ orbitals, and the bands of interest (text) involve the $h_{9 / 2}$ and $h_{11 / 2}$ orbitals.

Fig. 2-11. The sclution of eq. (2-2) for the $h_{9 / 2}$ orbital for $\lambda=-2.5 \mathrm{MeV}$, i.e., in the $s_{1 / 2}$ and $d_{3 / 2}$ levels. The dots correspond to the experimental levels seen in ${ }^{199}$ Tl and ${ }^{179}$ Re, placed at a value of $\beta$ taken from the neighboring doubly-even core nuclei. The dashed lines correspond to solutions of the extended Hamiltonian with $\mathrm{a} B$ and $\mathrm{C}$ term; the values of $B$ and $C$ are obtained by fitting the lowest levels of the neighboring core nuclei.

Fig. 2-12. A comparison of the observed negative-parity levels in ${ }^{199}$ Tl with those calculated by eq. (2-2) and by the extended Hamiltonian with a B and C term. All parameters determined from ${ }^{198} \mathrm{Hg}$. The dashed levels are tentative.

Fig. 2-13. The solution to eq. (2-2) for the $h_{11 / 2}$ orbital with $\lambda$ of $2.0 \mathrm{MeV}$ for ${ }^{195} \mathrm{Au} \quad$ s) and at $1.0 \mathrm{MeV}$ for ${ }^{179} \mathrm{Re}$ (botton). The dots show the experimental level energies.

Fig. 2-14. A comparison of the obst ved negative-parity levels in 195 fu with those calculated by eq. (2-2) and by the extended Hamiltonian. 
$-5 E-$

L.8L-2346

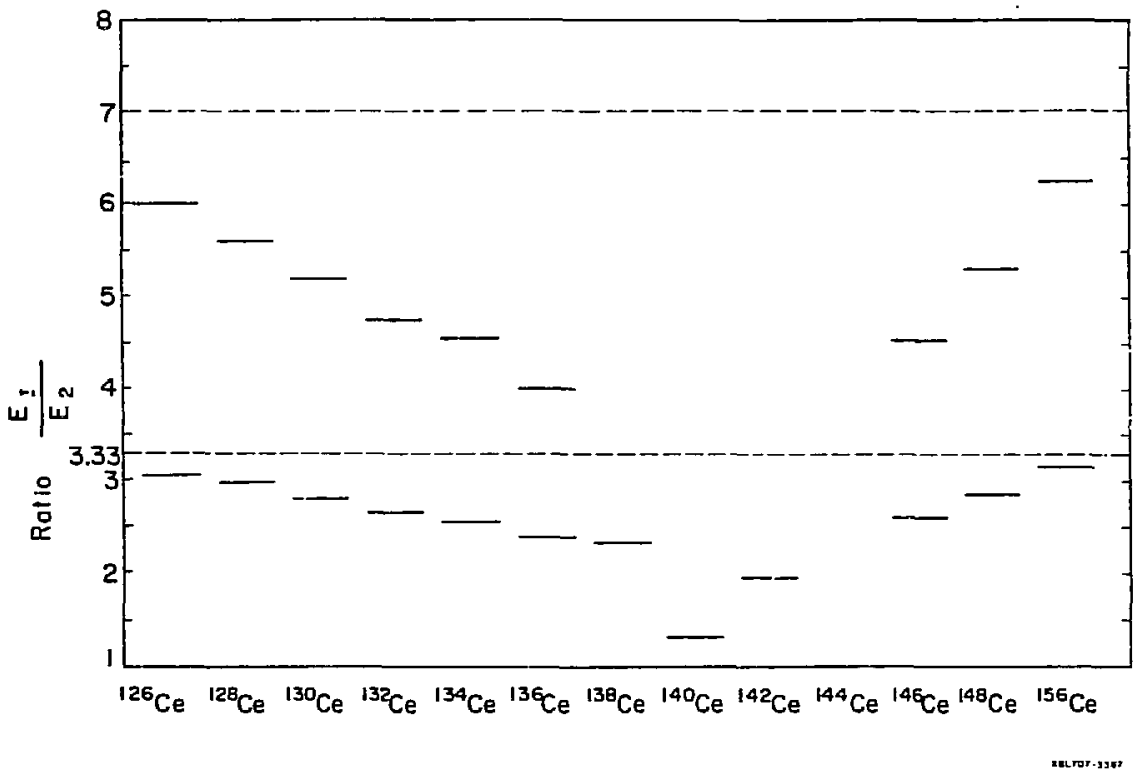

Fig. 2-1 

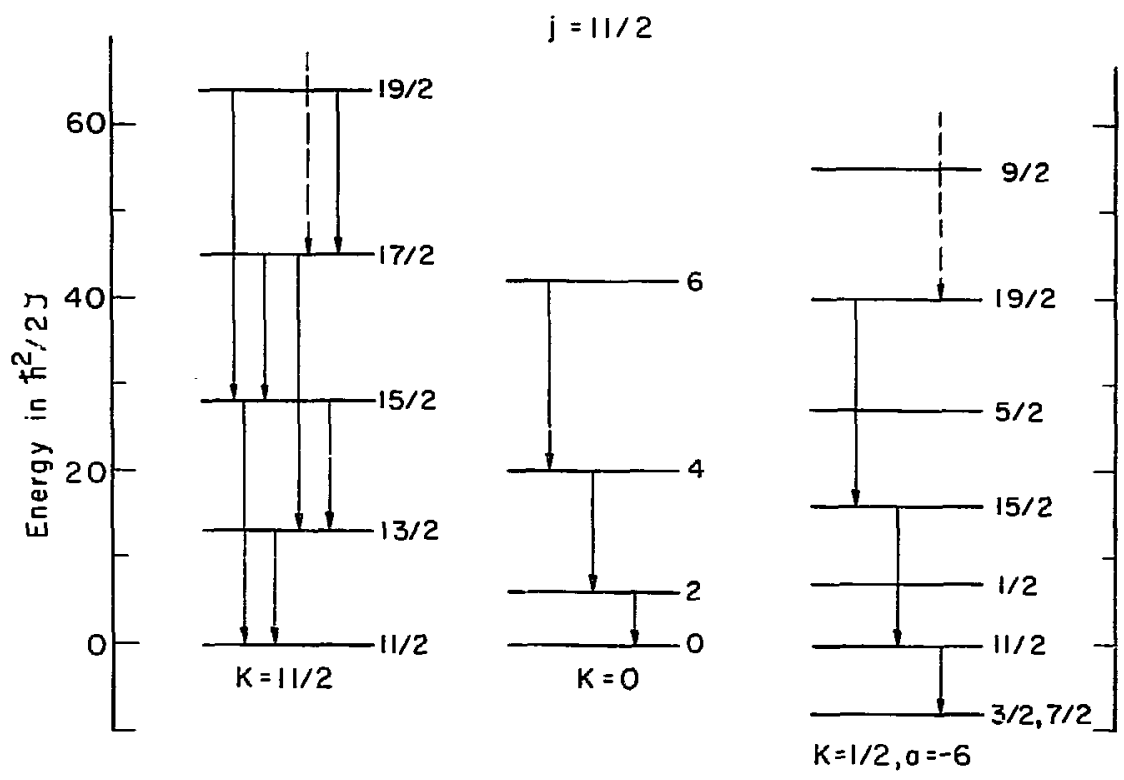

XBL725-3024

Fig. 2-2 


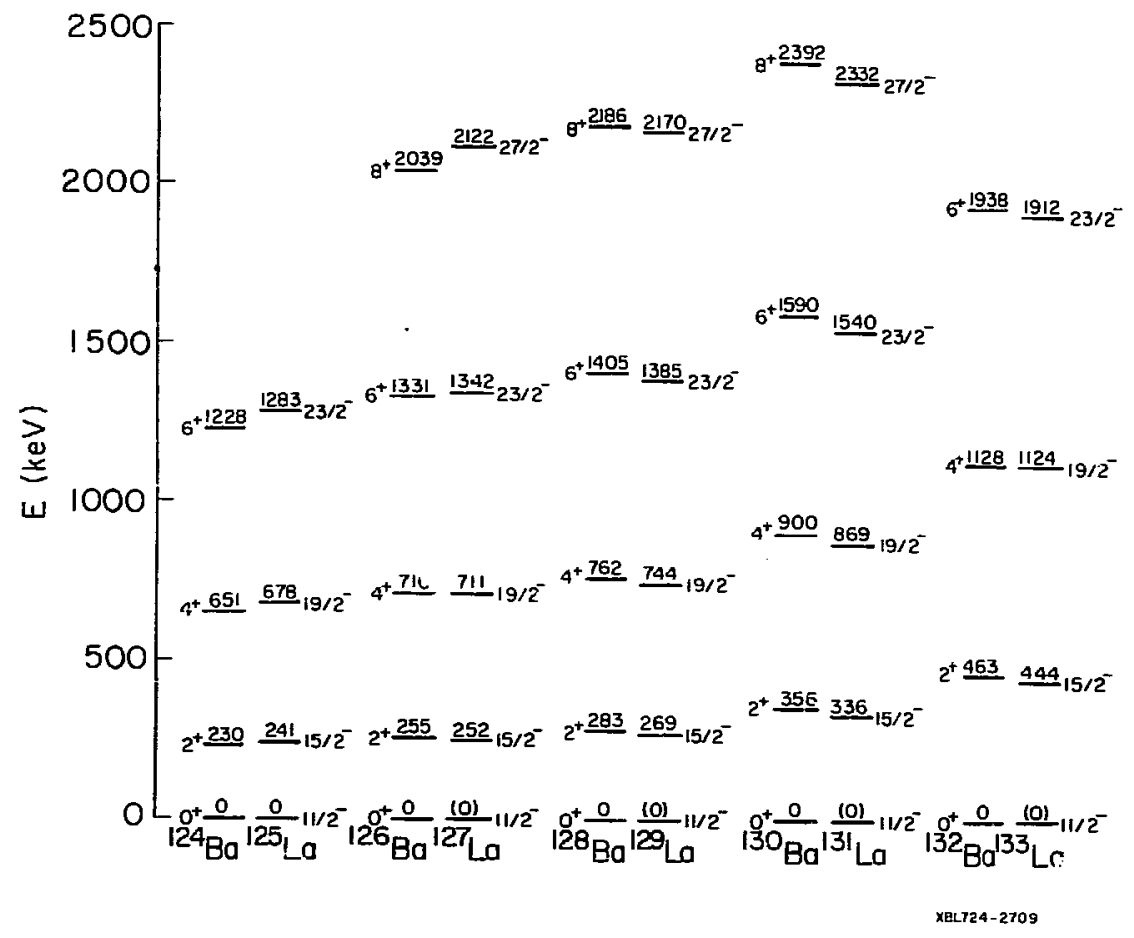

Fig. 2-3 

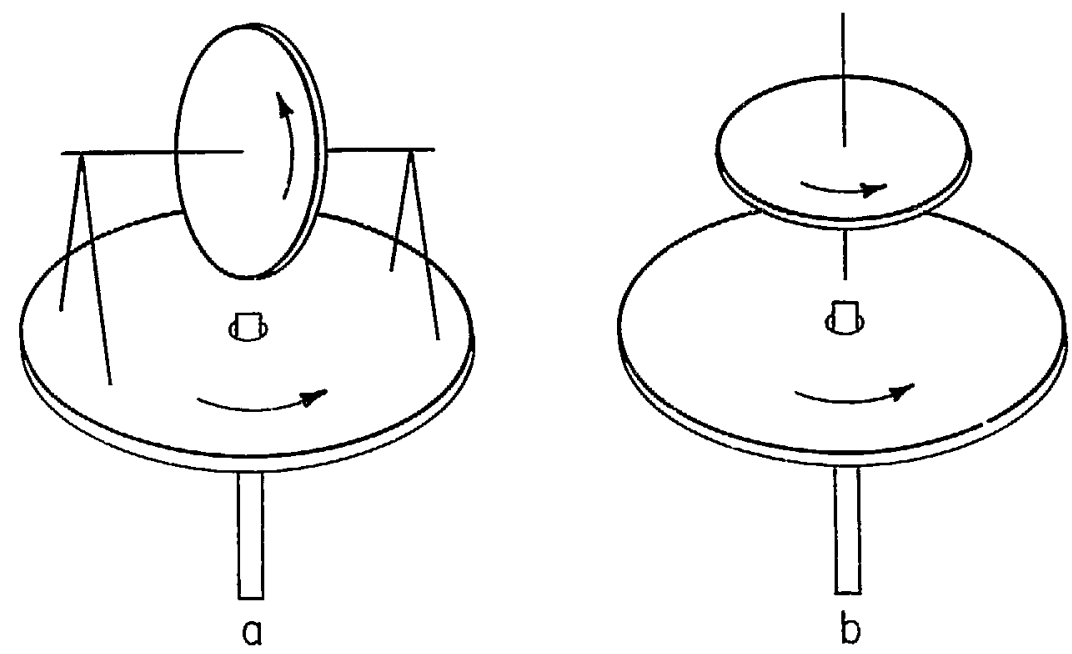

XBL7110 -4458

Fig. 2-4 


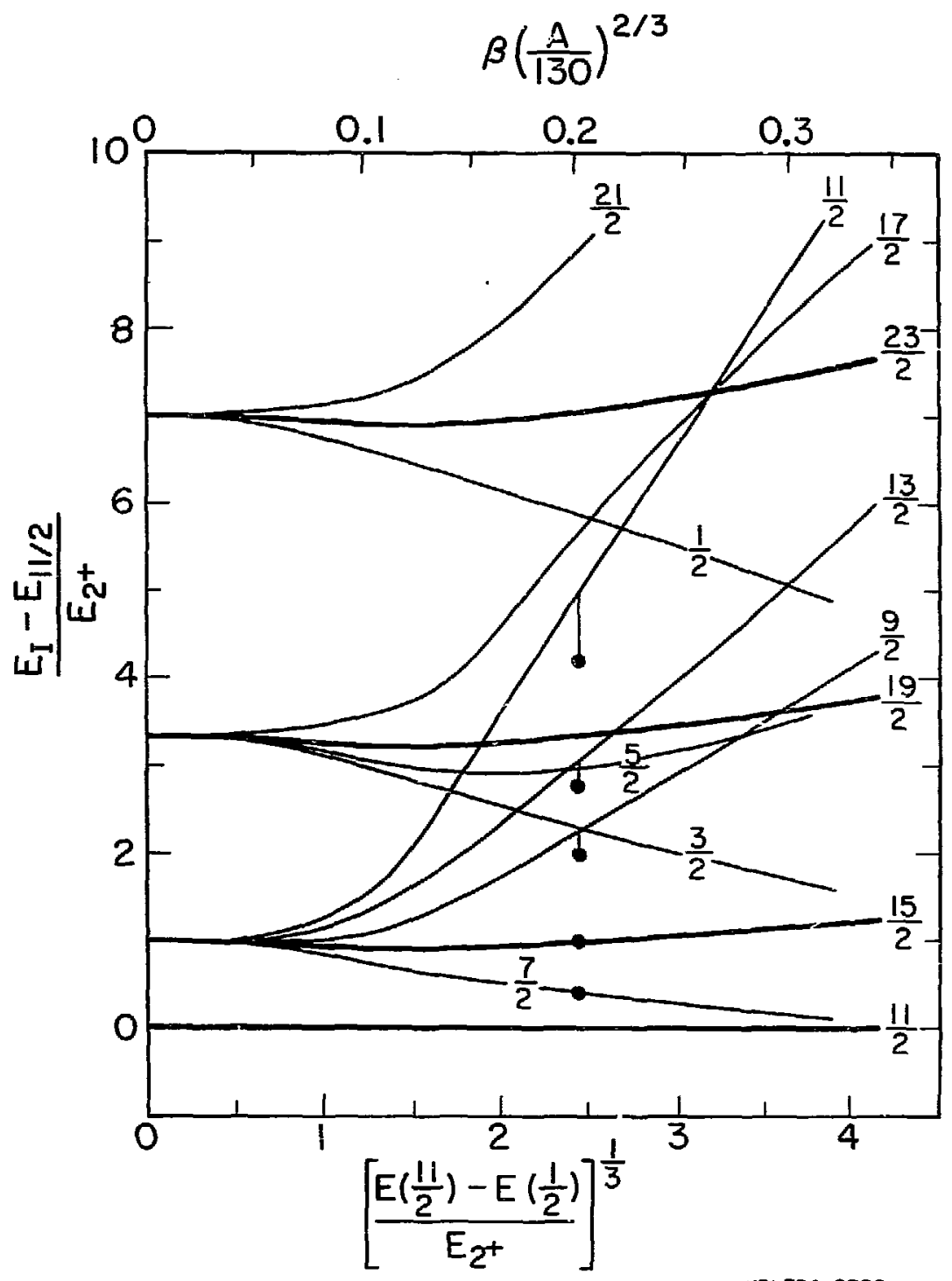

X日L724-2708

Fig. 2-5 


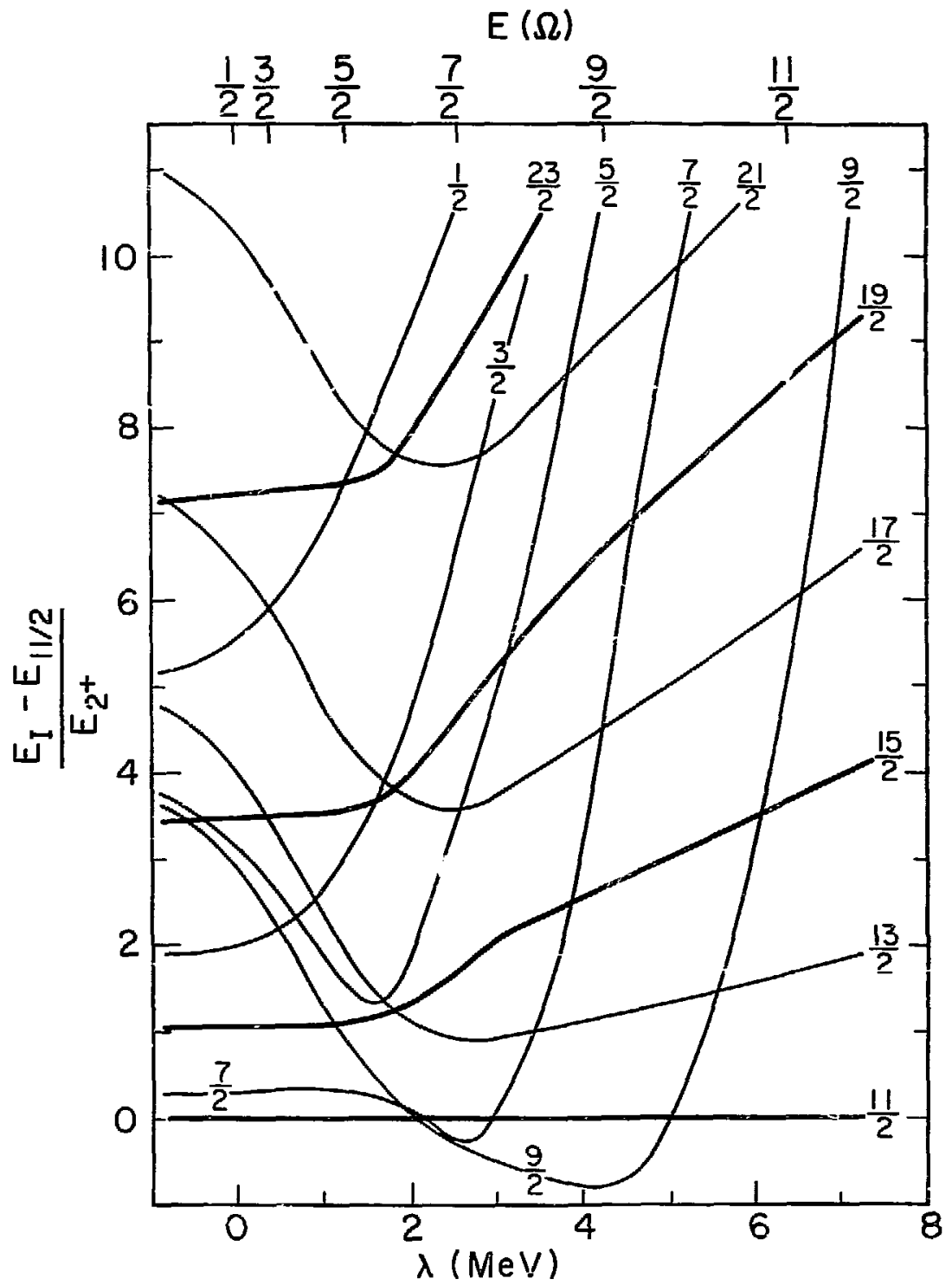

XBL724-2707

Fig. 2-6 

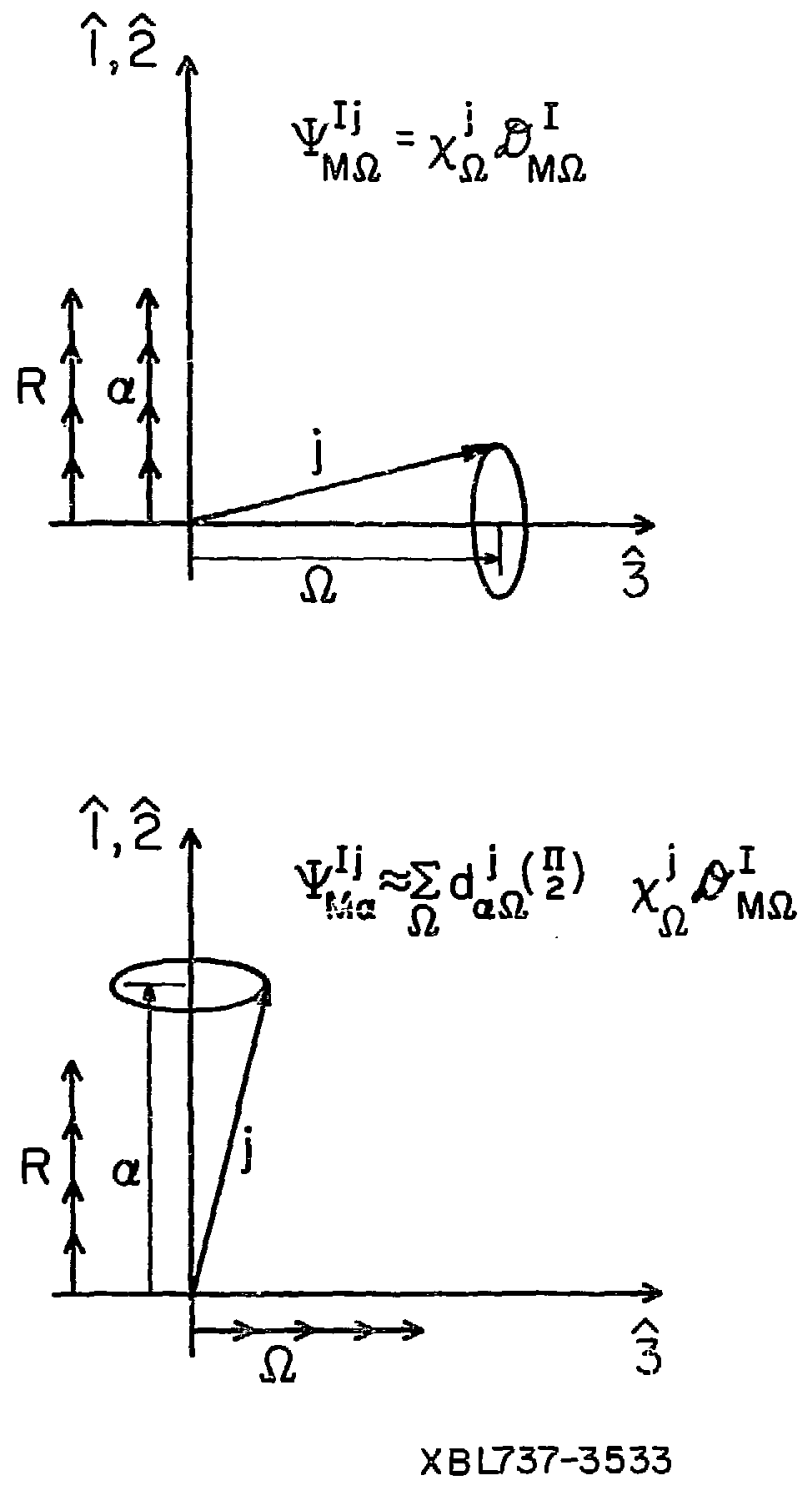

Fig. 2-7 

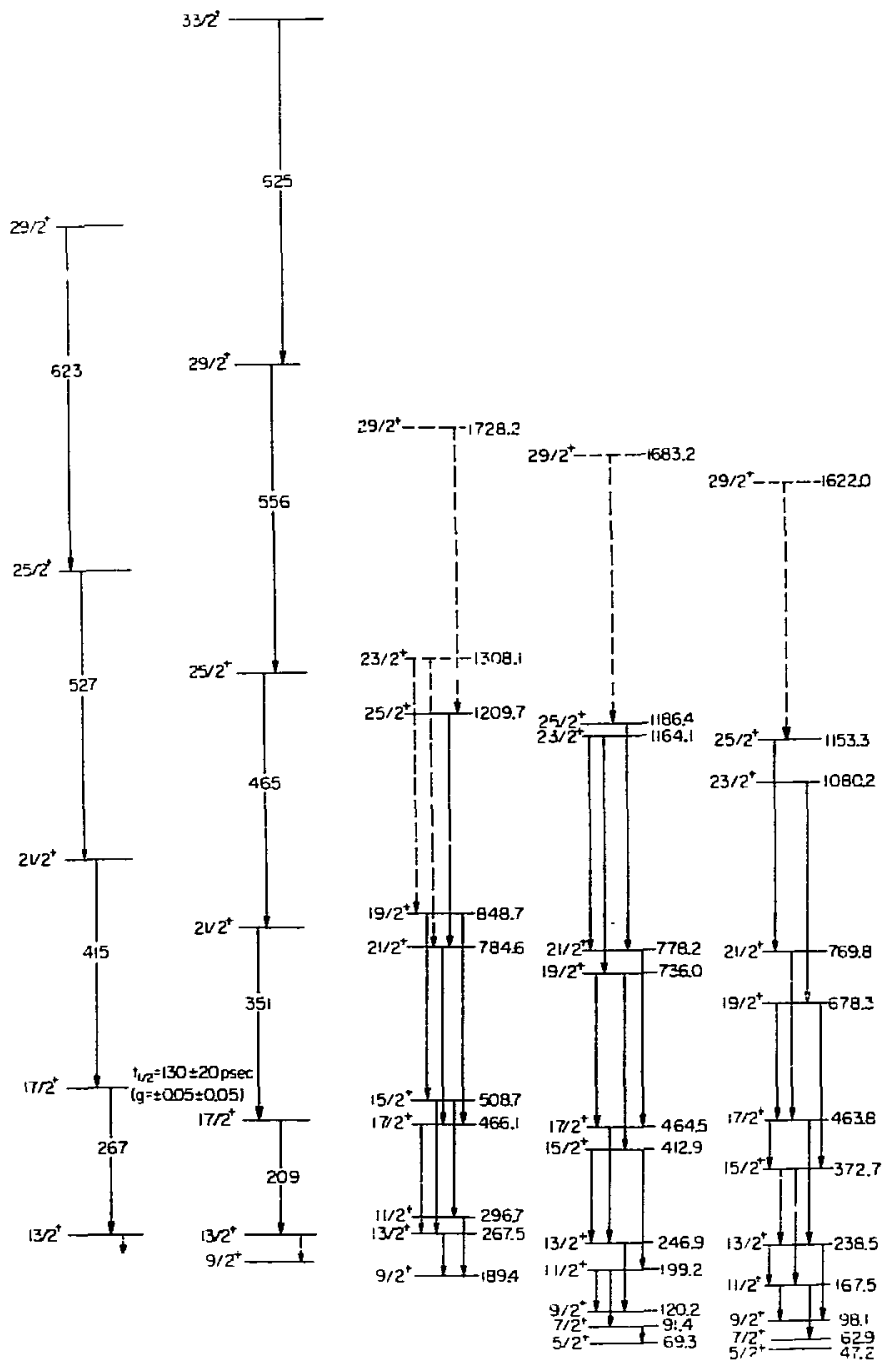
${ }^{157} \mathrm{Er}$
${ }^{159} \mathrm{Er}$

${ }^{|6|} \mathrm{Er}$

${ }^{163} \mathrm{Er}$

${ }^{165} \mathrm{Er}_{\mathrm{r}}$

xat $\bmod -3634$

Fig. 2-9 
$-64-$

$L B L-23 \triangle 6$

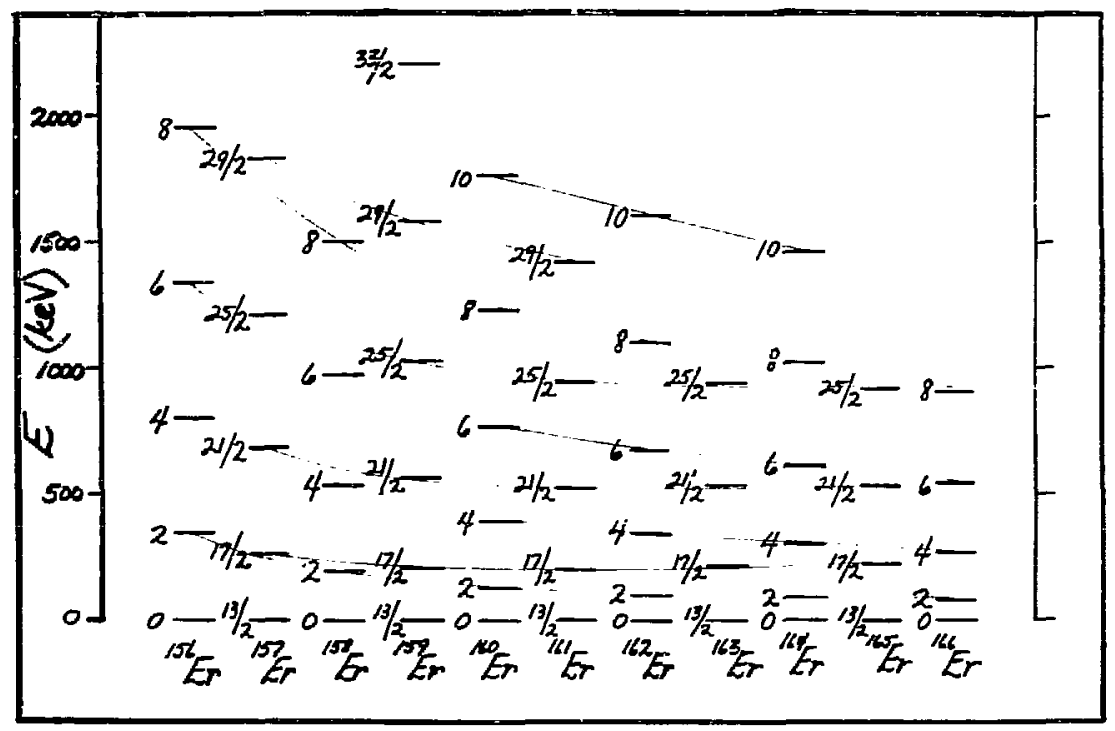

X日L $725-3026$

Fig. 2-9 


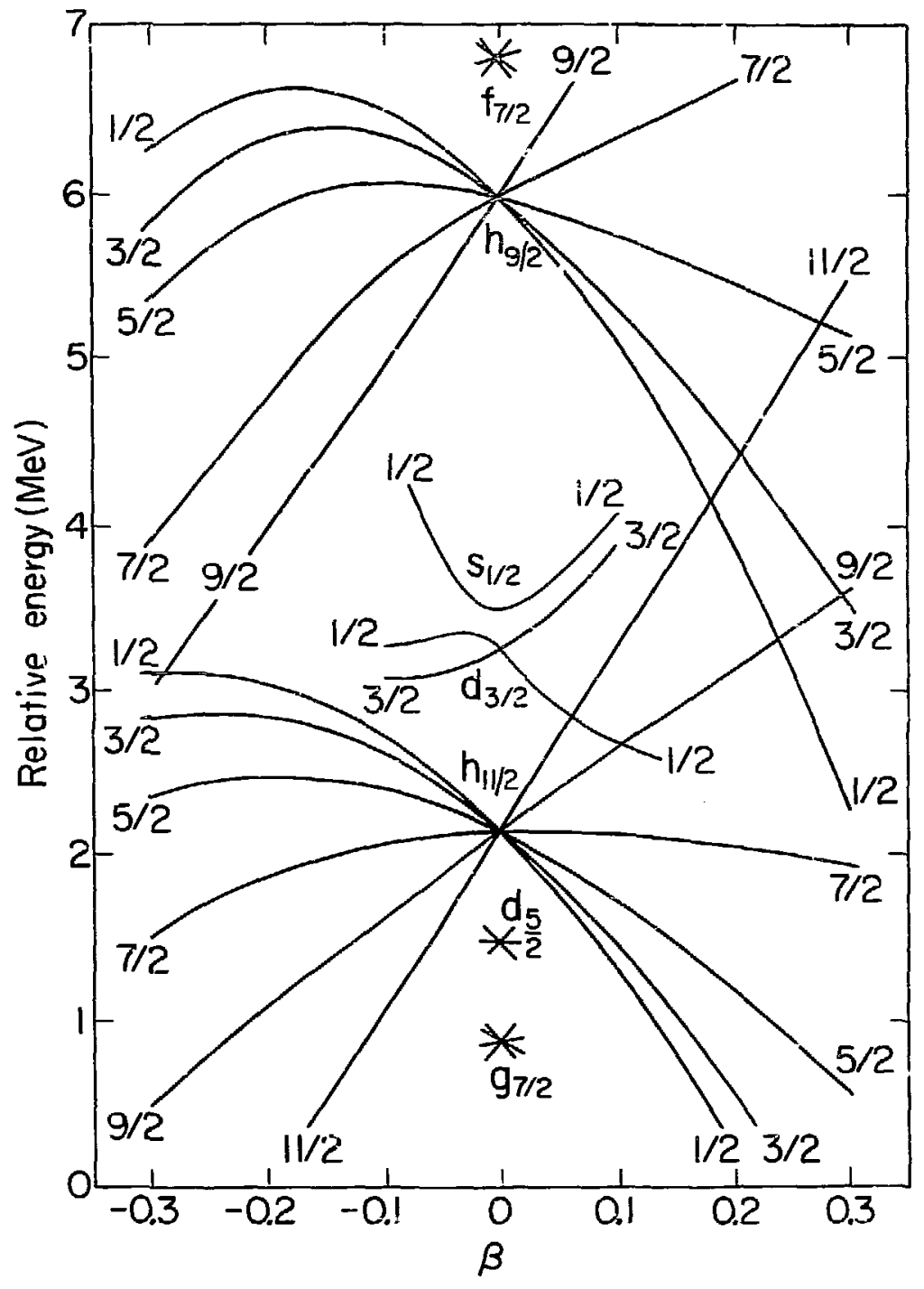

XBL725-2887 


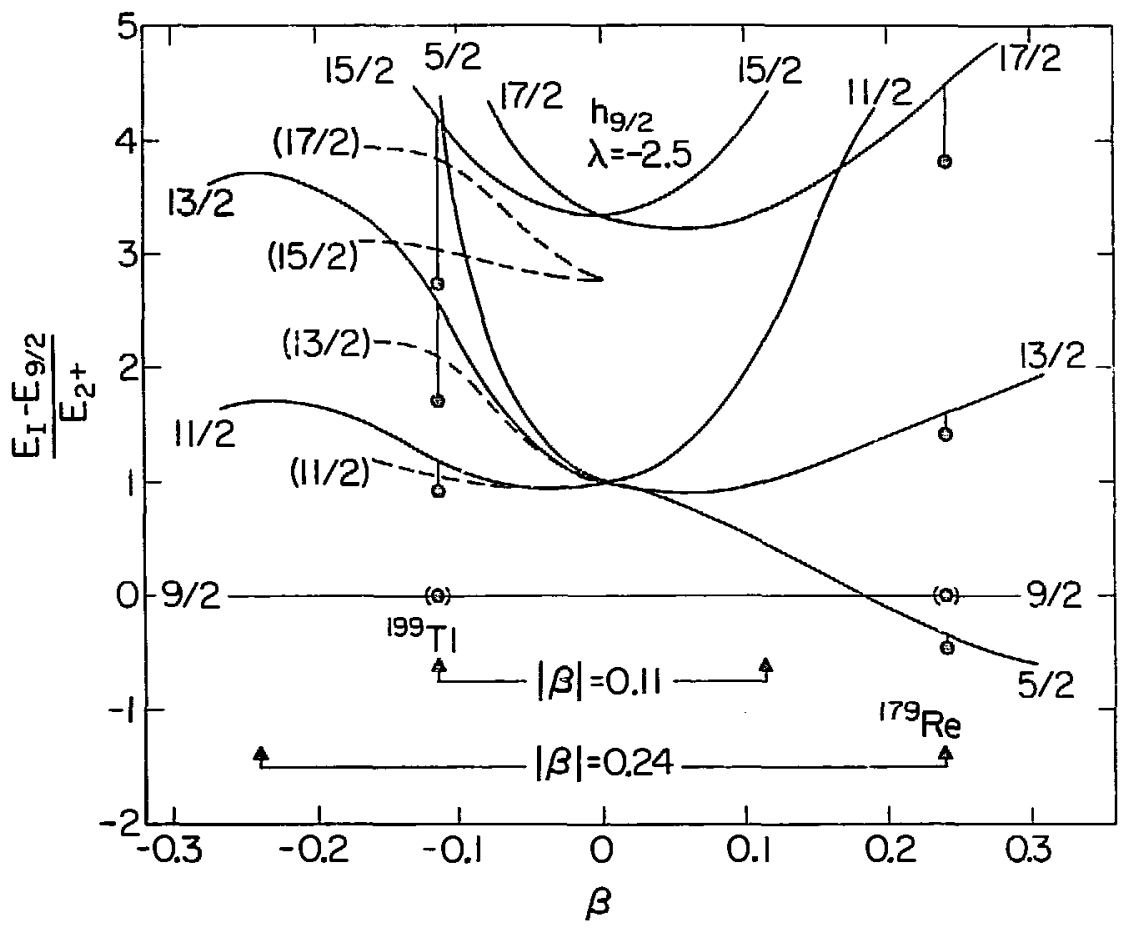

XBL 725-2888

Fig. 2-11 


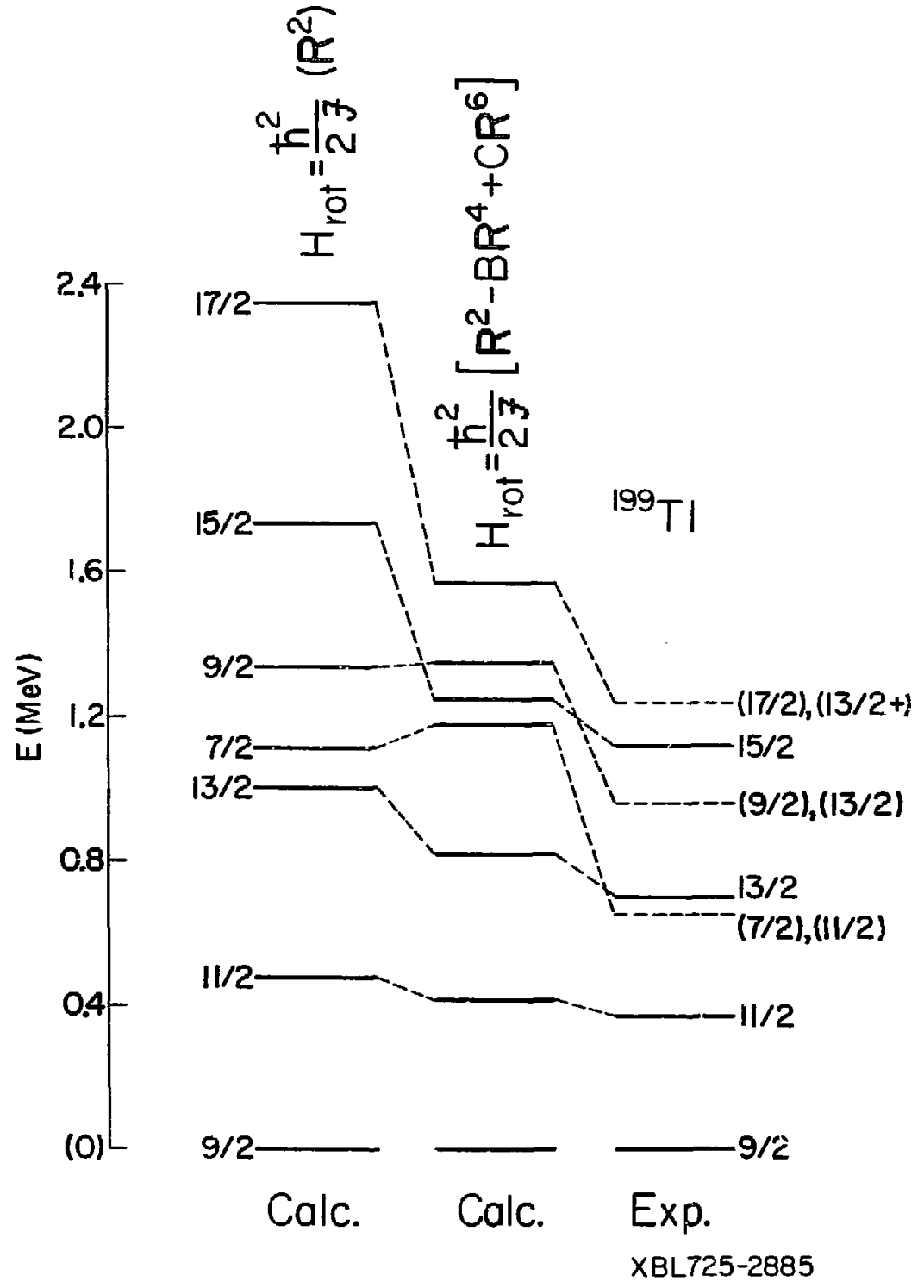

Fig. 2-12 


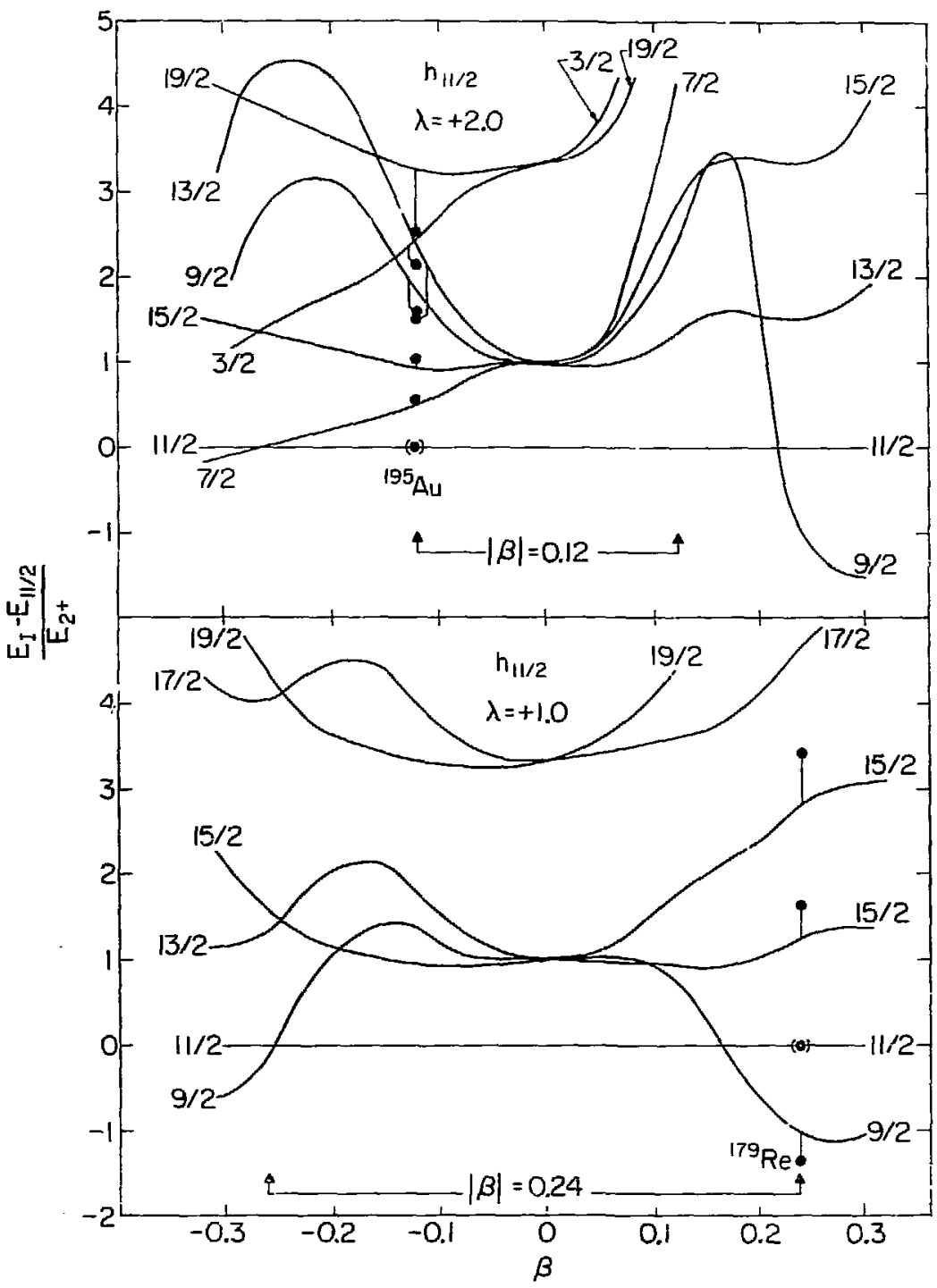

XBL 725-2889

Eị̦. . 2-13 


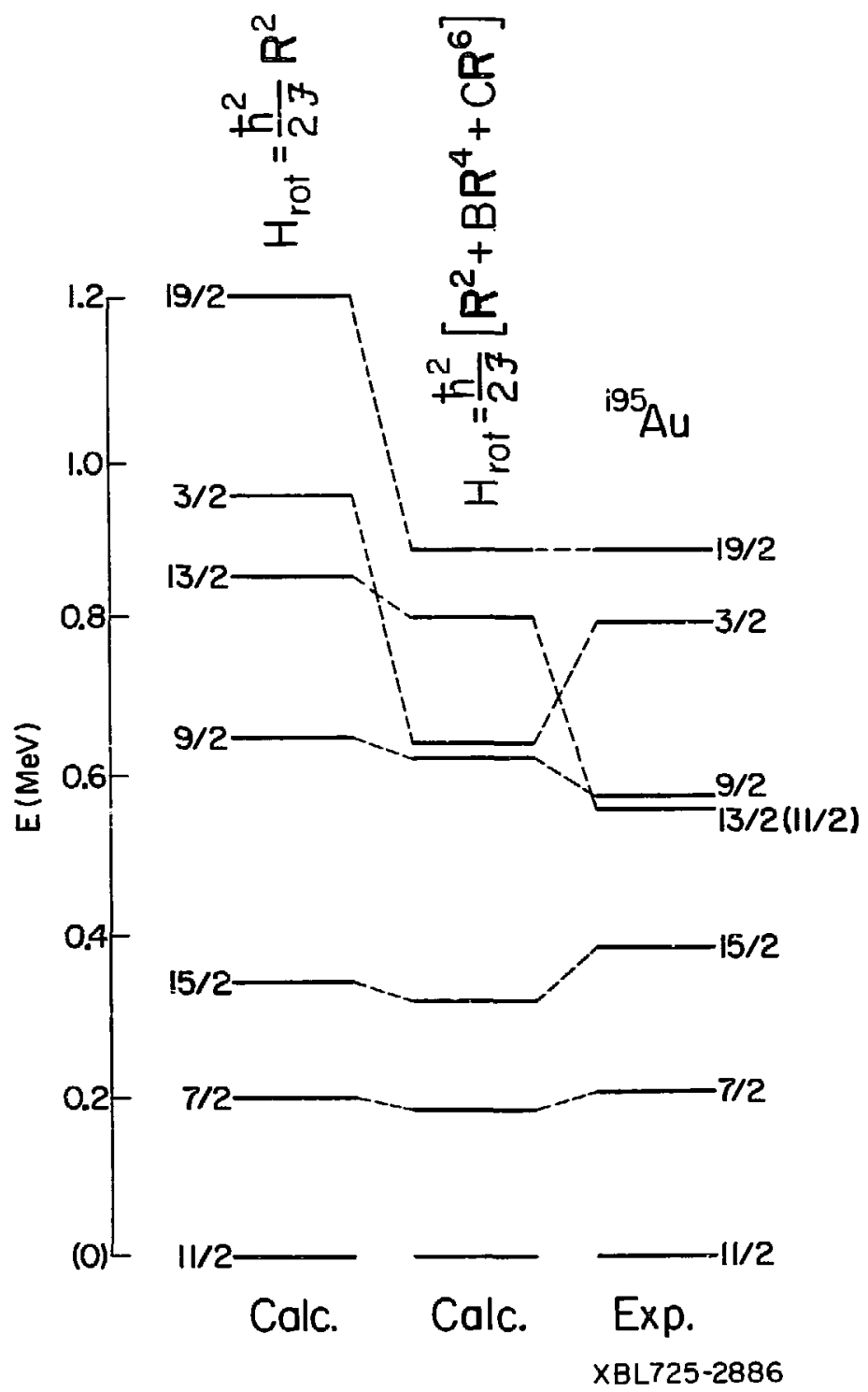


Part III. Coulomb Excitation")

3-1. Introduction and General comments

Today I'd like to talk about Coulomb excitation using heavy ions. BY heavy ions, I mean everything from ${ }^{4}$ He on. As already mentioned, heavy ions have some important uses in scattering and reaction processes. They bring in a large amount of angular momentum. I talked a little about one aspect of this feature in the first lecture (Part I). Another property is that they have a high charge. The most important use of the high charge is in multiple coulomb excitation, which I shall discuss this time and next. A third feature is that heavy ions bring in a large linear momentum. This can be usefully applied, as with the recoil-distance Doppler-shift method of measuring lifetimes in the range of $10^{-10}-10^{-12}$ seconds. I hope to describe this method to you if there is time. In this lecture on Coulomb excitation, I am not going into the methematical details, because there are many good reviews on the subject, but I would like to give you an idea of what the process is and describe some of the difficulties in doing an experiment.

What is coulomb excitation? If a projectile bombards a target nucleus at an energy below their mutual coulomb barrier, it will be repelled by the nuclear charge of the target and will scatter elastically. This is Rutherford scattering. But there is a small probabiifty that one or the other of the nuclei will beccae excited in the collision via the rapidly changing electromagnetic field between them; the excitation energy is taken from their relative kinetic energy. This is coulomb excitation. What can we get out of coulomb

\footnotetext{
*) Part of this lecture was given by the author at the Internation conference on Nuclear Mcments and Nuclear Structure, Osaka, 1972. For further studies of coulomb excitation, see references cited in Proc. of Conf. II-e; J. Phys. Soc. Japan, Suppl. 34118 (1973).
} 
excitation? We can directly get the energies of the excited states (sometimes the deexcitation transition energies are the primary data), and from a little bit of the for alism we can get the spins and parities of these levels, if the ground-state values are known. Perhaps the most impcrtant quantities obtained from Coulomb excitation measurenents are static and transition electric moments (in this case the magnetic excitations are much weaker). In particular, quadrupole excitations have been studied most thoroughly, so I will spend most time on them.

Let us consider what is important in coulomb excitation, looking at Fig. 3-1. We have a deformed target nucleus and a projectile with charges $z_{2}$ and $z_{1}$, respectively. The projectile is assumed to be a point charge; this is what is ustially assumed, but is only a convenience in calculation, leading to a monopole-multipole expansion, and is not a handicap of the theory. The monopole-monopole repulsion term determines the hyperbolic scattering orbit. But when we consider the charge distribution of the target nucleus (static or dynamic), the repulsion which is proportional to $1 / x^{2}$ gives a net torque to the target nucleus and makes it rotate. Let us consider the times involved in the excitation. The time of collision $\left(T_{C}\right)$ is given roughly by

$$
\mathbf{T}_{\mathbf{c}}=\frac{2 \pi \mathrm{a}}{\mathbf{v}},
$$

where the distance of closest approach is $2 a_{\text {i }}$

$$
a=\frac{z_{1} z_{2} e^{2}}{m v^{2}},
$$

and $v$ is the velocity of the projectile. The nuclear excitation time (T exc) is defined as 


$$
T_{\text {exc }}=\frac{2 \pi \hbar}{\Delta E},
$$

$\Delta \mathrm{E}$ being the nuclear excitation energy. The ratio of these two is given by the symbol $\xi$, which is often called the adiabaticity paraneter,

$$
\xi=\frac{T_{c}}{T_{\text {exc }}}=\frac{a \Delta E}{\hbar v}=\frac{z_{1} z_{2} e^{2} \Delta E}{h_{m v}{ }^{3}}
$$

If the collision time is longer than the nuclear excitation time, then the target nucleus is merely going to follow the projectile. That is, the symetry axis of the deformed nucleus keeps a direction at right angles to the line from the projectile. No impulse is given the target nucleus, so coulomb excitation cannot occur in this adiabatic case. However, if the collision time is short compared with the nuclear excitation time, the particle goes by very rapidly and the nucleus cannot respond immediately but feels the torcue. Afterwards it starts to rotate or vibrate. Therefore, from the above discussion, the parameter $\xi$ must be less than one if there is to be appreciable coulomb excitation. For a particular target-projectile combination, the only way to make this parameter small is to make the velocity large, that is, to make the bombarding energy high. However, this brings another problem. If the energy is too high, the projectile comes so close to the target nucleus that nuclear interactions occur and destroy the advantage and simplicity of the pure coulamb-excitation process. I forgot to mention earlier that the beauty of coulomb excitation is that it yields the very important set of nuclear parameters, the electric moments, without recourse to nuclear theory. If only the well-understood electromagnetic interaction is is be involved, the excitation must occur at an energy far enough 
below the barrier to guarantee that there is no nuclear interference; the Coulomb repulsion must be strong enough to keep the projectile far enough away from the target nucleus to allow only electromagnetic excitation. So another important condition is that the quantity $n$, defined as*

$$
\eta=\frac{z_{1} z_{2} e^{2}}{h v},
$$

must be such as to keep the particle far away from the target. 'hat is, $\mathrm{n}$ has to be mucin greater than one. To make this number large we have to make the velocity small. So, there is a conflict between the conditions on the two parameters $\xi$ and $n$. This point is something which has to be recognized when we set up a particular experiment. The particle velocity or energy should be as high as possible to obtain a good yield, but it cannot be above a "safe" energy where the interaction is still purely Coulombic.

In most cases, calculations of coulomb excitation are done in a semiclassical approximation where the basic assumption is that the orbit is the classical orbit (classical hyperbola). Such a classical approximation is valid under the two conditions $\xi \ll 1$ and $n \gg 1$, because under these conditions the ratio

$$
\frac{\Delta E}{E}=\frac{\Delta E}{\frac{m v^{2}}{2}=\frac{2 \xi}{n} \ll 1,}
$$

and the incident particles do lose only a very small fraction of energy, so that they do not change their classical orbit.

*) The quantity $n$ is the ratio between $a$, half the distance of closest approach, and the compton wave length of the projectile $\lambda(=h / m v)$, that is $\Pi=a / \lambda$. 
Coulomb excitation also depends on the charge of the projectile, since a and $\eta$ depend on $z_{1} z_{2}$. If the charge is higher, the particle gives a bigger torque to the nucleus. So, that is the advantage of heavy ions. Also, the larger the moment of the target, the more probable the excitation. For a more quantitative description of the effects of projectile velocity and charge on the coulamb excitation cross section, I refer you to the standard texts. If protons are used as the projectile, the probability of coulonb excitation is small since the charge of the proton is not large. Then, for even-even nuclei, only the first $2^{+}$state can be obtained by a single-step quadrupole excitation, and for a deformed odd-mass nucleus, the '-wo lowest states with spins one and two units above that of the ground state can be excited. That is all that can be done with protons, but these were very important experiments. There are regions of the Periodic Table where the energy of the first $2^{+}$state falls very low, and Coulomb excitation by protens and alphas showed that the reduced transition moments become very large, of the order of 100-200 single-particle units. The observation of such enormous values in the middle of the rare-earth region was one of the first triumphs of the Coulomb excitation process, and was a beautiful illustration of the rotational model of Bohr and Mottelson. But it was very limited after a few years, when the first $2^{+}$state in most even-even nuclei had been excited. Now, with the use of heavy ians the probability of coulomb excitation goes up, and we come to the possibility of multiple coulonb excitation, where several quanta of excitation are given to the target nucleus rather than just one. Multiple excitation is still a single-collision process, but one in which 
a large range of states of various spins are produced. This certainly is a more interesting possibility, but it also makes the problem more complicated. For example, if we excite the $14^{+}$state by a seven-fold excitation, as we have done in ${ }^{238} \mathrm{U}$ with ${ }^{40}$ Ar projectiles, at least seven transition muments are involved in the excitation instead of one. These transition moments are reflected in the yield of the various states; the larger the reduced moment, the larger the yield. So, the important experimental problem is to measure the yields of the states that are coulomb excited. There are two general techniques for doing this. One is to measure the yield of scattered particles with a spectrograph or a simple colid-state detector. Say we are using ${ }^{4}$ He particles; mosi of the alphas are elastically scattered, but a small percentage have a lower energy. The difference in energy from the elastically-scattered particles is the energy of the coulomb-excited stace, the $2^{+}$state in an even-even target. The ratio of the yields of these inelastic events to the elastic ones gives an absolute measurement of the probabiiity of coulomb-exciting the state. This simplicity is the great beauty of determining the particle yields.

Another way is to look not at the projectiles, but at the $\gamma$-rays deexciting the states produced. This procedure is not as straightforward. When looking at $\gamma$-rays, we have to worry about where they come from, because we get $\gamma$-rays from a state not only by coulomb excitation of that state, but also by feeding from higher states. The most severe problem with $\gamma$-ray analysis is that we dor't have any simple way to normalize the yields to Putherford scattering. As every measurement must be an absolute measurement, we have to determine the efficiency and the geometry of the detector, the beam current, and the angular distribution of the $\gamma$-rays. If the target nuclei 
recoil into vacuum from a thin target, then the attenuation of the $\gamma$-ray angular distribution by the hyperfine field from any unpaired electrons (mainly s-electrons) on the recoiling nucleus must also be determined. In addition, the modes of decay of the state of interest must be known or cetermined in order to obtain absolute yields. However, there are also some advantages to $\gamma$-ray detection. One can get a high counting rate with $\gamma$-ray detection but not so easily with particle detection. And the energy resolutior. can be as good as $2 \mathrm{keV}$. Good energy resolution with a particle-detection method is limited by the homogeneity of the beam energy and the much pocrer resolution of particle detectors. The most serious limitation with particle detection of heavy-ion beams is the problem of the target itself. Because it must have a finite thickness to yield adequate statistics, the energy spread in the target may be quite severe. For example, let us consider a $10 \mu \mathrm{g} / \mathrm{cm}^{2} \mathrm{Sm}$ target. This is a pretty thin target, but for back-scattered $10 \mathrm{MeV} \alpha ' s$ it will contribute an energy spread of $4 \mathrm{keV}$. If we go to a 100 $\mathrm{MeV}{ }^{40} \mathrm{Ar}$ beam, this becomes $160 \mathrm{keV}$, greater than the energy of the first excited state in a deformed nucleus. And for a $330 \mathrm{MeV}$ Xe beam, the spread due to the target becomes $570 \mathrm{keV}$. With $\gamma$-ray measurements, the energy resolution is not a function of the projectile mass or energy except for a possible Doppler shift for very fast transitions. Obviously, there a e advantages and disadvantages in both schemes, but with very heavy ions, it will probably be easier to work with $\gamma$-rays. For light ions such as protons or $\alpha$ 's, the particle detection method seems superior. A method which is often used that combines some of the best features of both methods, especially for multiple coulomb excitation studies, is to observe the $\gamma$-rays in coincidence with 
back-scattered projectiles. Counting the scattered particles gives the excitation probability, and the back-scattered projectile has the largest probability for multiple excitation, as it goes in closest to the target nucleus.

I'd like to take up one more problem irivolved in doing coulomb excitation work before going to some examples if experimental results. It has been mentioned already that the collision must not involve nuclear interactions. That is really important. We can only use Coulonb excitation theory to extract answers from the data if the reaction has been done at a low-enough energy to be truly free of nuclear interferences. WE can determine the safe-energy region where only coulomb excitation occurs in several ways. For example, when only coulomb excitation occurs, we can calculate the probability of the excitation as a function of energy from the transition probability, as will be shown below. We then take the computed ratio, ${ }_{\text {comp' }}$, between the yield of the $2^{+}$state and that of the Rutherford scattering,

$$
\mathbf{R}=\frac{\sigma\left(2^{+}\right. \text {state) }}{\sigma \text { (Rutherford scattering) }}
$$

and compare with the experimental ratio, $R_{\exp }$. If nuclear interactions are not involved in the process, the double ratio $\mathrm{R}_{\exp } / \mathrm{R}_{\text {comp }}$ should be unity. Figure 3-2 shows an example for ${ }^{4} \mathrm{He}$ end ${ }^{16} 0$ bombarding ${ }^{114} \mathrm{Cd}$. In the ${ }^{4} \mathrm{He}$ case, there is a little dip at about $10 \mathrm{MeV}$ and the double ratio goes up very rapidly afterwards as you increase the incident energy. The dip is caused by interference with nuclear interactions, and the sharp rise afterwaras is the dominance by nuclear inelastic scattering at higher energies. In the ${ }^{25} 0$ case, 
we can also see a slight dip at about $44 \mathrm{MeV}$. So, for Sm targets we have to do experiments beiow $10 \mathrm{MeV}$ with ${ }^{4} \mathrm{He}$ and below $44 \mathrm{MeV}$ with ${ }^{16} 0$. When we want to know whether we are in a safe-energy region or not, we have to do some such experiment as the one shown, or compare elastic scattering to Rutherford. A number of experiments of this nature have been done, and result is a maximum safe bombarding energy given by the following equation:

$$
E_{\max } \frac{1.44 z_{1} z_{2} e^{2}}{\left.E_{1}+R_{2}\right)+t}\left(1+m_{1} / m_{2}\right)
$$

where $\mathrm{R}$ and in are the zadius and the mass, and subscripts 1 and 2 mean projectile and target, respectively. According to this formula, the nuclei must be kept a distance apart of the order of magritude of $t$. The problem is what to use for the distance $t$ and the radius $R$. If $R$ is chosen as $r_{0} A^{1 / 3}$ with $r_{0}=1.2 \mathrm{fm}$, then $t$ is 5-6 fm. Figure 3-3 illustrates another way to evaluate the maximum safe energy from a determination of $t$ in eq. (3-8).- In it, the effective values of the static quadrupole moments measured by nuclear reorientation for ${ }^{48} \mathrm{Ti},{ }^{56} \mathrm{Fe}$ and ${ }^{60} \mathrm{Ni}$ are plotted as a function of the projectile energy expressed in terms of the parameter $t$. In this case, nuclear interference dramatically affects the apparent value of the quadrupole moments. As you san see, $t=5 \mathrm{fm}$ roughly gives the maximum safe energy.

3-2. Experimental Results of Coulomb Excitation

The beauty of Coulomb excitation is that it is a well understood phenomenon from which you can get very important nuclear guantities, namely the nuclear moments. Actually, ali you measure are electric moments, because 
magnetic excitation is smaller than electric excitation by a factor of $v^{2} / c^{2}$. Now, when you have measured the yield of a particular excited state, say the yield of the $2^{+}$state in an even-even nucleus bombarded by protons, how do you extract the nuclear moment from this yield? Witis such a low-z projectile only a single-step Coulomb excitation can occur. In a single-step process, it is easy to get the relationship between yield and $B(E \lambda)$, because there is a one-to-one correspondence given by first-order perturbation theory,

$$
\sigma_{E \lambda}=\frac{z_{1}^{2} A_{1}}{40}\left[0.072\left(1+A_{1} / A_{2}\right) z_{1} z_{2}\right]^{2 \lambda_{1}+2} E^{\lambda-2}(E-\Delta E)^{\lambda-1}{ }_{B}(E \lambda) f_{E \lambda}(\eta, \xi) .
$$

Subscripts 1 and 2 are projectile and target, respectively. The term $\left(1+A_{1} / A_{2}\right)$ is the recoil effect. Because $\Delta E$ is usualiy smell compared with $E$, the yield is proportional to $\mathrm{E}^{2 \lambda-3}$, and in the case of $\mathrm{E} 2$ excitation, propoitional simply to $E \cdot f_{E \lambda}(\eta, \xi)$. The last quantity, $f_{E \lambda}(\eta, \xi)$, is a function of the two coulomb excitation parameters, the adiabaticity parameter $\xi$ and the somerfeld parameter n. To obtain the value of $f_{E \lambda}(\pi, \xi)$, integrals over the path of the projectile must be carried out. These integrals have been calculated by Alder and Winther and tabulated. For differential cross sections, the same type of expression results, but $f(\xi, \eta)$ is replacea by $\mathrm{d} f(\xi, \eta, \theta)$. The straightforward way to get. a $B(E \bar{L})$ value for excitation of the first $2^{+}$state in an even-even nucleus is to pick conditions such that only a single-step excitation occurs. Then all parameters are known but the $B(E 2)$, and that is determined if you measure the cross section. Thus, it is still important to do single-step excitation studies because we need accurate values of these transition moments for many purposes. 
However, the usual coulomb excitation processes employed today are multiple excitation processes, in which more than one quantum is transferred in a single collision, yielding higher states, and higher-spin states. Now, how äo you obtain $B(E 2)$ values from such measurements? One could use higher-order perturbation theory, i.e., $2^{\text {nd }}$ order for the $4^{+}+2^{+}$ transition, $3^{\text {rd }}$ order theory for the $6^{+}+4^{+}$, etc. But what is usually done is to use the computer code written by deBoer and Winther, which gives a semi-classical solution to the system of coupled differential equations. For detailed descriptions, see the references. The point is that, for this calculation, you need a lot of input data for the computcr code. You must put in all the matrix elements connecting all the states that may be involved in the excitation, including higher states in the same band, and states of comparable spin in other (vibrational) bands that can decay to the initial state of interest. You also have to consider the possible importance of higher multipole transitions, such as hexadecapole, and virtual excitation through highlying states such as the giant dipole resonance. All these transition matrix elements, including an estimate of the ones being measured, are put into the computer code, and it calculates the resulting cross sections and angular distributions. These can be compared with the experimental results, and by a series of enlightened tries, agreement is soon reached. Obviously, if any of the important matrix elements in the calculation are omitted or are incorrect, the rrong value(s) will be obtained for the matrix element(s) being determined. Clearly, most or the important matrix elements in the calculation, other than the one or ones being determined, must be obtained from independent experiments or from model calculations. In addition, the effects of vacuum polarization 
and electron screening of the projectile and target may be considered. Fortunately, they are both 1 or smaller and opposite in their effects, so for most experiments, where the accuracy is a few percent (or worse) it is all right to forget about them. More important are quantal corrections to the semi-classical computer code usually used, and I shall come back to this topic later.

3-2-1. Energy Levels and B(E2) Values

Now, let us talk about results. One of the earliest and most exciting results of Coulomb excitation was to show that in certain regions of the periodic table, mainly in the rare-earths and actinides, where the first excited states of even-even nuclei lie very low, the $B(E 2)$ values connecting the first $2^{+}$states and the ground states are eery large. Values reached 100 or even 200 single-particle units, and this was the best confirmation at that time of the collective rotational model of Bohr and Mottelson. Such large values could only occur if many nucleons were involved. There is a simple expression for the energy of a rigid rotor,

$$
E_{I}=A I(I+1)
$$

This equation fits surprisingly well for the spectrum of low-Iying states for nuclei in the middle of the rare-earth and actinide regions. However, as the energies of higher stater in the ground band were determined by multiple Coulomb excitation, $\beta$-decay, and $\alpha$-decay studies, it turned out that Eq. $(3-10)$ fit more and more poorly. So additional correction terms were added. The first correction to Eq. (3-10) has the form of a rotation-vibration coupling, 
which is well known in molecular spectroscopy. With this term, the energy is given by

$$
E_{I}=A I(I+1)+\mathrm{BI}^{2}(I+1)^{2}
$$

and the second tera shows the initial deviation from the rigid-rotor model values.

Then, what about the deviations of the values of $B(E 2)$ from those of the rigid-rotor model? In that model, the reduced quadrupole transition probabilities are related to each other through the following equation,

$$
\mathrm{B}(E 2 ; I+I-2)=\mathrm{B}(E 2 ; 2+0) \frac{(I 020 \mid I-20)^{2}}{(2020 \mid 20)^{2}}
$$

So, an interesting study is to see whether the experimental values follow the above expression or whether they also deviate from the rigid-rotor value. And if so, do they deviate in the same sense as the energies?

First of all, I would like to point out that it is a much more difficult problem to determine transition probabilities than energies. It is easy with a Ge detector to measure energies to $1 / 10$ percent. But, to measure B(E2) values to $1 / 10$ percent, the yields must be determined to $1 / 10$ percent, and I don't know anybody who does that. Obtaining coulomb excitation yields to a few percent requires a careful experiment. Since it can be expected that the deviations in the transition probabilities might be about the same order of magnitude as the energy deviations, you can see that it is much more difficult to measure the former, and requires going to higher spins where the deviations are probably larger. If there are deviations, we want to be able to parametrize them in some convenient manner. If the cause is a rotation-vibration interaction, a 
mixing with the $\beta$-band and/or $\gamma$-band, you can treat this two-band mixing problem and obtain the following modification of Eq. (3-12),

$B(E 2 ; I \rightarrow I-2)=B(E 2 ; 2 \rightarrow 0) \frac{\langle I 020| I-20)^{2}}{(2020 \mid 20)^{2}} \times\left\{\frac{1+(\alpha / 2)[I(I+1)+(I-2)(I-1)]}{1+3 \alpha}\right\}^{2}$

where $\alpha$ is the mixing parameter or the stretching parameter, as it appears from either theory. However, I do not intend to imply any special significance about the physical meaning of $\alpha$, but only to use the quantity as a measure of the deviations from the rigid-rotor transition probabilities. . Fhen ... = 0 , Eq. (3-13) becomes just the rigid-rotor case; when $\alpha>0$ the $B(E 2)$ 's get larger with increasing spin, and when $\alpha<0$ they get smaller as I increases. (A) $166_{\text {Er and }} 172,174,176 \mathrm{yb}$ nuclei

A number of multiple Coulomb excitation experiments have been done to try to measure how the ground-state $B(E 2)^{\prime} s$ change. Table 3-1 gives the results of a study done by Sayer, Stelson, et al. at Oak Ridge. The nclei in the table are all in the well-deformed region of the rare-earths, and are found to be good rotors. Coulamb-excitation yields were measured up through the $8^{+}$states, and the corresponding values of $B(E 2)$. were calculated by means of the deBoer-Winther computer program. The values of $\alpha$ derived from the B(E2;'s are shown in the table and they average about $-(1.4 \pm 1.0) \times 10^{-3}$. The negative values of $\alpha$ mean that the values of $B(E 2)$ get smaller going up the band to higher spins and it corresponds to centrifugal shrinking.

If we believe the simple two-band mixing model, $\alpha$ is also related to the rigid-rotor energy-level deviations by

$$
\alpha=-B / A,
$$


where $B$ and $A$ are given in $\mathrm{Eg}$. (3-11). So, another estimate of $\alpha$ can be abtained from the energy splittings. The values of $\alpha$ so derived are slso listed in Table $3-1$ and they are all positive and small, averaging $\sim+(0.5 \pm 1.0) \times 10^{-3}$. Why does this value not agree with the value from the $B(E 2) ' s$ ? We might have hoped that these two sets of values for $\alpha$ would be similar, at least that they would have the same sign. The origin of this problem appears to be the use of the semi-classical computer code of deBoer-Winther without making guantal corrections.

Although $n$ was large $(n=50)$ in the ${ }^{16} 0$ exci.tations, and this fact would indicate that the classical calculations were valid, the large value of $\eta$ only says that the first-order excitation from $0^{+}$to $2^{+}$is classical. It does not say what happens to the $6^{+}$to $8^{+}$transition which is a higher-order transition. In fact, the quantal corrections go up strongly with the multiple order of the Coulonb excitation. And most unfortunately, these corrections have not all been calculated yet. They have been calculated and tabulated in some special cases only through second order, and at the osaka Conference there will be a report from the Pittsburgh group about a third-order calculation up to the $6^{+}$level. The only thing we can do at the present moment is to estimate these quantal corrections by seeing how the corrections scale from $0^{+}$ to $2^{+}$to $4^{+}$. Such an estimate shows that the quantal corrections in the present case wipe out the negative values of $\alpha$ given in Table $3-1$, bringing the average value of $\alpha$ from the B(E2) measurements to zero. So, this estimate brings essential agreement between the two values of $\alpha$ calculated from the energy-level spacings and from the $B(E 2)$ studies, and the nuclei listed in Table 3-3 are well-described as good rotors with very small values of $\alpha$. 
(B) $150 \mathrm{Nd}, 152 \mathrm{Am}$ and ${ }^{154} \mathrm{Gd}$ nuclei

One way to better observe deviations is to go to nuclei which have larger values of $\alpha$. Iarger values of $-B / A$ are shown by the 90-neutron nuclei, ${ }^{150} \mathrm{Nd},{ }^{152} \mathrm{Sm},{ }^{154} \mathrm{Gd}$, that mark the onset of deformation in the rare-earth region. These nuclei have very low-lying $\beta$-bands at $\sim 680 \mathrm{keV}$, and it turns out that the most important mixing into the ground band is with $\beta$-bands. This mixing corresponds to centrifugal stretching, so we do expect much larger values of $\alpha$ for these cases. Experimental values of $\alpha$ for ${ }^{152} \mathrm{sm}$ are tabu. 7 ted in Table 3-2. The first three values are from multiple coulomb excitation experiments done by groups at Yale, Oak Ridge, and our group at Berkeley. Within the experimental errors, I think there is fair agreement among these three, but this may be somewhat illusory, as it is not clear whether or not the various groups have treated the corrections in the same way, and have used the same number of states in the calculation. In fact, I believe the original data may differ more than the final results. I shall describe below the way we treated the data, but first let me mention the two other values of $\alpha$ listed in the table.

The fourth value of $\alpha$ is determined from the energy-level spacings. As you can see, it is much bigger than the first three values. This suggests that there are more features involved in making the energy levels deviate than in causing deviations in the transition probabilities. The last value of $\alpha$ was determined by a different kind of experiment, a recoil-distance Doppler-shift measurement. In this measurmment, Coulomb excitation is used to create the excited levels, but Coulomb excitation theory is not involved in the deduction 
of the $B(E 2)$ values; the lifetimes of the states are directly measured. I cannot discuss this experiment in this lecture because of time limitations, but I think that in favorable cases this method probably gives more accurate values of $\alpha$ than straight multiple coulomb excitation experiments.

Now, the states we took into account in our calculations are shown in Fig. 3-4. We are interested in looking at the ground-band $B(E 2 j ' s$ up to the $\mathrm{B}^{+}$state, so we should include at least one state beyond; we took two. That is, in the ground band we included states up to the $12^{+}$. But we must also include states in other bands that can feed into the ground band up to the $8^{+}$ level. In particular, we must consider the $\beta$-band up to the $12^{+}$state, the $\gamma$-band up to the $12^{+}$state, the $\mathrm{K}=0$ octupole band, and the $\mathrm{K}=1$ octupole band. There are probably stıll more bands, but since we did not see any significantly intense unidentified transitions in the experiments, they are most likely not important. For the calculation we need to use all the matrix elements connecting all the states shown in Fig. 3-4. Many of these are unknown, and had to be estinated. But all these E2 and E3 matrix elements require a very large computer program. We extended the deBoer-Winther program to 20 states, but that was still not enough. We had to break up the input data into sections, and then make separate runs to show that these results can be added.

How big the corrections are is shown in Table 3-3. The fractional corrections to the $4^{+}+2^{+}, 6^{+} \rightarrow 4^{+}$and $8^{+} \rightarrow 6^{+}$yields are listed in the table in percent. They include the effect of the direct excitation of the ground-state band through that band, and the $Y$-cascade from the band to the ground band. The $\beta$-band only introduces a $1 \%$ change. The $\gamma$-band, $\mathrm{K}=0$ cctupole band, and 
$K=1$ octupole band have slightly larger effects of either sign. The most important corrections in this case are for the E4 moments; this subject will be discussed in greater detail below (3-2-2). The total corrections range from 5\% to $25 \%$. But how many corrections are missing? I do not know. Certainly quantal corrections should be included, and they will be negative and several $\%$ in magnitude. The last line shows the change in $\alpha$ due to the corrections listed. If we did not include such corrections in the analysis, we would get a larger $\left.\alpha^{\star}\right)$ than the one listed in Table 3-2. I do not believe the other groups have made the same corrections for $\alpha$ when they deduced the values of $\alpha$ listed in Table 3-2, so the apparent agreement there may not be real.

Figure 3-5 shows the actual $\gamma$-ray spectra for ${ }^{16} \mathrm{O}$ and ${ }^{40} \mathrm{Ar}$ excitation of ${ }^{152}$ Sm. With ${ }^{4}$ He projectiles the $2^{+}$state is mainly excited, just rarely the $4^{+}$. With ${ }^{16} 0$ on ${ }^{152} \mathrm{Sm}$ the $2^{+}, 4^{+}$and $6^{+}$states can be excited, and with ${ }^{40} \mathrm{Ar}$ projectiles, the $2^{+}, 4^{+}, 6^{+}, 8^{+}$, and $10^{+}$states, as shown in the figure. In the last case, the product - $n$ of the low-spin states is not by direct excitation, but mainly by cascade decay from the higher-spin states. I might also note in passing that compared with heavy-ion reaction spectra, you obtain very clean and simple spectra by coulomb excitation.

\section{3-2-2. E4 moments}

As you see in Table 3-3, the largest corrections to the yields were due to the hexadecapole moment. From the results of a study of Sm Coulomb excitation with ${ }^{4} \mathrm{He},{ }^{16} \mathrm{O}$, and ${ }^{40} \mathrm{Ax}$ beams performed several years ago, we were *) Note that the experimental value of $\alpha$ listed in Table 3-2 is $\alpha_{\text {obs }}$ (uncorrected) $-\Delta \alpha$, where $\alpha_{\text {obs }}$ (uncorrected) is that deduced from Eq. (3-13) and $\Delta \alpha$ is the correction for a given on the last line in Table 3-3. 
forced to the conclusion that the E4 moment was large. What happened was that we obtained different $B(E 2)$ values, different values of $\alpha$, from excitation of the $2^{+}$and $4^{+}$states with the different projectiles. For example, the value of $\alpha$ with ${ }^{40}$ Ar excitation was somewhat smaller than with ${ }^{16} 0$ excitation (even negative for the ${ }^{40} \mathrm{Ar}$ case), and the value of $\alpha$ with ${ }^{4} \mathrm{He}$ was much larger than the ${ }^{16} 0$ value by considerably more than the statistical errors. It seemed something was missing in the calculation. We did not know which calcusation was wrong, that for the ${ }^{4} \mathrm{He}$ case or that for the ${ }^{16} 0$ case. To solve this problem, an independent experiment must be done to determine what the true $B(E 2)$ is.

This is the reason why we started the recoil-distance Doppler-shift measurements for $152,154 \mathrm{Sm}$. This technique does not involve multiple coulomb excitation theory, and I think, in principle, such measurenents are a more straightforward way to determine actual lifetimes. Since we know that these transitiors are pure E2, the lifetimes give the values of $B(E 2)$. That experiment gave us the answer that $\alpha$ for ${ }^{152} \operatorname{Sm}$ is $(1.9 \pm 0.6) \times 10^{-3}$ which was very close to the value for the ${ }^{16} 0$ case in Table 3-2. Actually, it was too close, as our ${ }^{16} 0$ valuz in the table will have to be raised by the quantal correction, probably about one unit in $\alpha$. But it was clear that the correct answer was close to the ${ }^{16} 0$ value, and so something was wrong with the 4 He calculation. We concluded that what was wrong was that we had ignored a significant E4 contribution to the excitation of the $\Delta^{+}$state when using the ${ }^{4}$ He excitation. This is shown in Fig. 3-6.

Most of the yield of the $4^{+}$state comes from double E2 excitation, (a), but there is also single-step excitation by the hexadecapole moment of ${ }^{152} \mathrm{Sm},(\mathrm{b})$, 
and an interference term, (c). To determine the $\mathrm{E} 4$ moment, one must measure the yield of the $4^{+}$state as carefuily as possible, and then subtract the effect of the double E2 excitation. The latter can be calculated if the $B(E 2)$ values are known accurately from careful measurements of the $2^{+}$yield, or from independent measurements such as the recoil-distance Loppler-shift technique. The remaining yield of the $4^{+}$state is then ascribed to the E4 excitation. The best projectile to use for such a study is ${ }^{4}$ He because the resulting double E2 excitation is not so strong as to bury the E4 excitation, but there is still sufficient excitation to observe the $4^{+}$state. The effect cannot be seen with ${ }^{16} 0$, because with ${ }^{16} 0$ projectiles there is so much multiple excitation that it completely dominates the picture; the fraction of direct E4 excitation is, I don't know exactly the calculated value, but maybe $10^{-4}$ or less.

What an E4 moment does to the cross section for excitation of the $4^{+}$ state $2{ }^{152} \mathrm{Sm}$ is shown in Fig. 3-7. This is a plot of the cross section with an E4 moment over the cross section with no $\mathrm{E4}$ moment against the reduced E4 matrix element as the abscissa. When this matrix element is 0 , the value 1 is abtained for the cross section ratio. The curve is a parabola; this comes from the fact that most of the yield due to tlie E$\leqslant$ matrix element comes from the direct E4 excitation which is proportional to the square of the matrix element. The weaker E4-double E2 interference term causes the asymmetry about zero. The important point is that we can measure the yield of the $4^{+}$state, calculate the ratio $\sigma / \sigma_{0}$, and then from this figure determine where we are on the plot, and hence the value of the E4 matrix element. 
Our measurements are shown in the following few figures. Figure 3-8 shows the $\gamma$-ray spectrum obtained with ${ }^{4}$ he on a natural $\mathrm{Sm}$ target. What we want to do is to compare the $y$ ields of the $4^{+} \rightarrow 2^{+}$transitions to the $x^{\text {ields }}$ of the $2^{+} \rightarrow 0^{+}$transitions, which are known quite accurately, to obtain absolute yields for the $4^{+}$levels. Knowing these yields and knowing the values of $\mathrm{B}\left(E 2 ; \mathrm{O}^{+}+2^{+}\right)$and $\mathrm{B}\left(E 2 ; 2^{+}+4^{+}\right)$, the computer code can be used to give the corresponding values of $\sigma_{0}$, and then the cross section ratio fixes the position on Fig. 3-7. We did both singles experiments and $\gamma$-coincidences with

-k-scattered particles for separated isotopes and for natural $\mathrm{Sm}$. The reason for doing both types of experiments is that there is a different dependence in these two experiments on the various states that have to be considered in the calculations. The contributions of these states to the fopulation of the $4^{+}$ state in ${ }^{252} \mathrm{Sm}$ by irradiation with $10.4 \mathrm{MeV}{ }^{4} \mathrm{He}$ are shown in table 3-4, where the effects of the $\beta$-band, the $\gamma$-band, the two octupole bands, and the $6^{+}$ level are listed. Column 5 shows the fraction of the decay which goes to the $4^{+}$level. The sixth and the last columns show the relative population of the $4^{+}$state contributed by that row to the singles and to the back-scattered cross sections, respectively. It can be seen that the proportions are different in the two cases.

Figure 3-9 shows the measured total (and differential) cross sections for the $4^{+}$ievel of ${ }^{152} \mathrm{Sm}$ divided by the appropriate calculated values with no $E 4$ moment as a furstion of the 4 ire bombarding eneigy. The triangles are the Eack-srattered-coincidence results and tile circles are the singles results. From the figure we see that both experiments give about the same answer for the increase of the $4^{+}$yield, approximately $11 \%$. From the figure it can also 
be seen that, if you go to higher energies, nuclear interactions take over, and the cross sections rise rapidly due to nuclear inelastic scattering. All the points above $11 \mathrm{MeV}$ are too high because of this effezt. So, you have to work at low energies. At low energies the yield is small, so that it takes a great deal of time to get adequate statistics. This is why the errors are so large in our experiments. The increase in the cross sections for the $4^{+}$ states of ${ }^{152} \mathrm{Sm}$ and ${ }^{154} \mathrm{Sm}$ that we observed were $\sim 11 \%$ and $\sim 12 \%$, respectively, and they yield ${ }^{*}$ )

$$
\left(4^{+} \|_{\left.M(E 4) \|_{0}\right)} \mid \begin{array}{l}
=0.45 \pm 0.09 \mathrm{eb}^{2} \text { for }{ }^{152} \mathrm{sm} \\
=0.67 \pm 0.08 \mathrm{eb}^{2} \text { for }{ }^{154} \mathrm{sm} .
\end{array}\right.
$$

These values are very large. The corresponding reduced transition probabilities approach the order of 50 single-particle units. So, these are not trivial matrix elements and they cannot be ignored in excitation calculations.

In Table 3-5 are listed our results and the large number of measurements done since them. The four independent measurements on ${ }^{152} \mathrm{Sm}$ and on ${ }^{154} \mathrm{Sm}$ are in fair agreement; experimentally the largest source of error comes from the two $\mathrm{B}(\mathrm{E} 2)$ values used in the double E2 excitation calculation. A small error in them makes a proportionately much larger change in the E4 matrix element. The major correction to the calculated cross section involves the quantal correction, and again the effect is magnified greatly, leading to a $30 \%$ increase in the Sm E4 matriy elenents. From the reduced E2 and E4 matrix elements, which are the primary results of the coulomb excitation measurements, one can

\footnotetext{
*) The corresponding negative matrix elements would be improbably large, and are in opposition to theoretical calculations for the nuclei.
} 
extract values of $B_{2}$ and $\beta_{4}$ with a number of assumptions. Some form must be assumed for the nuclear charge distribution, for example, a uniformly charged, axially symetric, rigid rotor with a radius given by

$$
R=R_{0}\left(1+B_{2} Y_{20}+B_{4} Y_{40}\right) \quad .
$$

A value of $R_{0}$ must also be assumed. If we take $R_{0}=1.2 \mathrm{~A}^{1 / 3}$ fm, we obtain the values, $B_{2}=+0.25$ and +0.26 and $B_{4}=+0.09$ and +0.13 for ${ }^{152} \mathrm{Sm}$ and ${ }^{154} \mathrm{Sm}$, respectively. For ${ }^{154} \mathrm{Sm}$ this leads to the shape shown in Fig. 3-10. But you must remember that this result very much depends upon the assumptions made, that is, it is model dependent. However, remembering this, the values of $\beta_{2}$ and $\mathrm{B}_{4}$ are of some interest to compare with the theoretical values derived from Nilsson's calculations and from the calculations of Kumar and Baranger, and also to compare with the results obtained in $\left(\alpha, \alpha^{\prime}\right)$ scattering experiments. The $\left(\alpha, \alpha^{\prime}\right)$ results give more directly the values of $\beta_{2}$ and $\beta_{4}$, but the analysis is still quite complicated, involving SWBA or coupled-channel calculations, and with some remaining ambiguity as to the magnitude of the nuclear radius, $R$. In addition, the results of the inelastic scattering have to do with the nuclear potential distribution, which may not be the same as the nuclear charge distribution.

\section{3-2-3. Static E2 moments}

Figure 3-11 shows pictorially several ways of ixciting the first $2^{+}$ state, corresponding to a perturbation treatment of the racitation. The upper figure (a) shows two kinds of processes, the direct excitation of the I $=2$ and $I_{z}=0$ state (first order excitation) and the second-order excitation in which some magnetic substate of the $I=2$ state is first excited but then drops 
to the state of $I=2$ and $I_{z}=0$. In addition to these first-order and second-order processes, we have the interference term between them, called the nuclear reorientation effect. The matrix elements involved in these processes are those for the $0^{+}+2^{+}$and $2^{+} \rightarrow 2^{+}$transitions; the latter is the static quadrupole matrix element of the $?^{+}$state. Thus, the resulting yield will be somewhat dependent upon the magnitude and sign of the static E2 matrix element. Actually, we also have other interference terms which complicate the measurement of the static quadrupole moment. One is the effect of higher $2^{+}$states. There is an interference term from this process (shown in the button-left drawing in Fig. 3-11), and the difficulty is that we don't know the relative signs of the matrix elements involved. There is also an interference term through the giant dipole $1^{-}$state. And there may be excitation by decay from the $4^{+}$states. So, sorting out the nuclear reorientation effect may be quite complicated. However, I shall perhaps stress the point that the usual reorientation effect is the interference between the first- and second-order processes, and is not the second-order process itself. The goal in setting up a reorientation experiment is to try to minimize all other interference effects, and there is an interesting contribution to the osaka Conference by Scharenberg et al., on how to do this by using low-energy very heavy ions.

The ratio of the nuclear renricstetion effect to the first-order excitation is given by perturbation theory as

$$
\frac{P_{i \rightarrow E}^{(12)}}{P_{i \rightarrow E}^{(11)}}=\frac{A_{1}}{z_{2}} \frac{\Delta E}{1+A_{1} / A_{2}}\left(2+\ln (E 2) \|_{2}+k(\xi, \theta)\right.
$$


Subscripts 1 and 2 refer to the projectile and target, respectively, $\Delta \mathbf{E}$ is the excitation energy, and the quantity $k(\xi, \theta)$ is a function which varies rather sharply with $\theta$, but only slowly with the velocity. We don't use this formula in an actual calculation, but rather the deBoer-Winther program, but it tells you what the reorientation process depends on. One way to measure the reorientation effect is to determine the $2^{+}$yield as a function of the projectile mass, because the ratio (3-16) changes with $A_{1}$. If the projectile is changed from ${ }^{4} \mathrm{He}$ to ${ }^{32} \mathrm{~s}$, the ratio $(3-16)$ is increased by a factor of 8 . Another way is to keep the projectile fixed, but measure the $2^{+}$yield as a function of the projectile scattering angle, since the value of $k(\xi, \theta)$ is largest for $180^{\circ}$ scattering and vanishingly small for forward scattering. I shall not go into the details of the calculation, both because of the limits of time and because there are a number of references on this topic. But to give you an idea of the order-of-magnitude of the effect, the contribution of the reorientation effect to the total yield of the $2^{+}$state in the case of ${ }^{16} 0$ excitation may be about $10 \%$. I would like now to show you some examples.

(A) $114 \mathrm{ca}$ nucleus

One of the earliest experiments of this nature was to measure the quadrupole moment of the first excited state of ${ }^{114} \mathrm{Cd}$, and that turned out to be a really exciting event. Because the ${ }^{114} \mathrm{Cd}$ nucleus is usually thought of as a "vibrator-type" nucleus, the quadrupole moment of the first excited state was expected to be small; it would be zero if it were truly a harmonic vibrator. However, the experiment gave the interesting result that the quadrupole moment was very large, that it was nearly equal to the rigid-rotor value calculated from the $0^{+} \rightarrow 2^{+} \mathrm{B}(\mathrm{E} 2)$. This caused a lot of excitement, so a number of groups 
began to measure this quadrupole moment using all possible variations of techniques, and theoreticians began to try to explain such a large moment. Table 3-6 shows a summary of recent experimental results. There is no consistency among all these data. The moment ranges from small positive $(+0.05)$ to large negative $(-0.85)$ values. So, something is wrong. A lot of people worried about what is wrong. We now know that the first three experiments are wrong because of the following reason. They were done by obtaining $2^{+}$yields from $\gamma$-rays coincident with back-scattered projectiles, using a light and a heavy particle, such as ${ }^{4}$ He and ${ }^{16} 0$. since the target is not thick, the excited nuclei recoil out of the target, and part of the time the decays originate from recoiling nuclei in vacuum. One of the important corrections to be made in obtaining absolute yields is for the $\gamma$-ray angular distribution. The first three experimental results were corrected for this effect, but what was not known at the time these experiments were done, is that there is a de-orientation of these recoiling nuclei, and that is really important. When they recoil into vacuum, the atoms are highly ionized and may have unpaired electrons (especially, s-electrons are important in this case). The unpaired electrons produce very large hyperfine fields (as high as 50 MGauss) at th: nucleus, and these randomly fluctuating fields destroy or attenuate the angular distribution of the $\gamma$-rays. If we take into account this effect on the $\gamma$-ray angular distribution, the first three values in Table 3-6 are decreesed by a factor of 2 , they should be $-(0.3-0.4)$ eb. Experiments directly comparing the intensities of the inelastic and elastic peaks at two angles have also given low values, but such measurements, which constitute the majority of the experiments in Table 3-6, have also given high values. How to explain that? Well, I don't know what the answer is. 
Certainly part of the discrepency among these data comes from the difference in the values of the B(E2)'s used. There is a $10 \%$ spread in the latter, which is just about enough to explain the deviations in the quadrupole moments. The independent measurement of the $\mathrm{B}(\mathrm{E} 2)$ for the $0^{+}+2^{+}$transition by $a$ different method, such as the recoil-distance Doppler-shift method, would help to solve this problem.

Anyway, I think the value of the quadrupole moment for ${ }^{114} \mathrm{Cd}$ is of the order of $-(0.3+0.4) \mathrm{eb}$. This is not as large as the rigid-rotor value, but it is still large. It is one of a number of examples that indicate that nuclei are not good harmonic oscillators. To explain this value you have to bring in either anhamonicity or asymetry of the nuclear potential. Tamura and udagawa published a paper years ago in which they showed a single way to create the anharmonicity by considering the mixing of the one- and two-phonon $2^{+}$states, and did obtain the right order-of-magnitude for the quadrupole moment. Last night Prof. Tamura told me that he has made an extension of the model which still gives the right quadrupole moment but yields a smaller value of the ratio $B(E 2 ; 2+0) / B(E 2 ; 2+2)$ which was a discrepancy with experiment in the first paper. This problem can also be looked at from the viewpoint of the triaxial model of Darydow and Chaban and Davydov and Ovcharenko to give the right magnitude and sign for the quadrupole moment.

(B) Nd, Sm and Ba nuclei

A much more expected result is shown in Fig. 3-12. These are the measured quadrupole moments for Nd and Sm. The sets of experimental results are in good agreement with each other. At $N=84$, the quadrupole moments are vanishingly small, as expected near a spherical closed shell. But, if 4 or 6 neutrons 
are added to the $\mathrm{N}=82$ core, larger moments result which are comparable to the rigid-rotor value. The additional neutrons really do appear to polarize the core, to deform the nucleus. In this region a deformation change is supposed to happen suddenly between $N=88$ and 90 , but these measurements seem to indicate a smooth transition.

On the neutron-deficient side from $N=82$, there shol:ld be the same effect, because neutron holes should polarize the core and increase the quadrupole moment just as do particles. Such a tendency can be seen from the open circles in the figure. But just before I left Berkeley, I received the Ba results shown by the filled circles, and these points lead to a worse situation than in the ca nuclei; they have the opposite sign to the earlier measurements. Obviously, there is something wrong. I cannot tell you what it is. This disagreement calls for a careful reinvestigation of the quadrupole mament in this region.

(c) Os and Pt nuclei

Figure 3-13 shows the quadrupole moments of Os and Pt done by the . Pittsburgh group of Dr. Saladin and his colleagues. This is a very pretty picture which shows a shape transition between Pt and Os. The lightest Os nuolet hawe targe prolate quadrupole monent and the heaviest Rt ones have an oblate nonbrit

(D) Iight nuclei by projectile reorientation

I am not going to have time to take up this subject in detail and show examples, but let me say just a few words about reorientation experiments (quadrupole-moment measurements) in the projectile. The ratio of the interference 
term to the first-order term in projectile excitation is given by an equation similax to Eq. (3-16):

$$
\frac{P_{i+f}^{(12)}}{P_{i+f}^{(11)}}=\frac{A_{2}}{Z_{1}} \frac{\Delta E}{1+A_{2} / A_{1}}\left(2^{+}\left\|_{M}(E 2)\right\|^{+}\right) k(\xi, \theta),
$$

where 1 and 2 again refer to the projectile and target, respectively. The ratio of the projectile effect to that of the target, Eq. (3-17)/Eq. (3-16), is proportional to $z_{2} / z_{1}$, for similar quadrupole moments. The atomic number of the projectile is usually $5-10$ and that of the target is $50-60$, so that the fifect of the interference term in projectile excitation is half-a-dozen times bigger than that in target excitation. Instead of a $10 \%$ effect with a target, there really is a 50 or $60 \%$ effect on the yield of the first $2^{+}$state in an even-even nucleus. This is, thexefore, much easier to do, and a goad way to measure quadrupole moments of light nuclei.

3-4. Possible future use of Coulomb excitation

Coulamb excitation studies provide very interesting and exciting results and have great possibilities in the future with heavy ions. One reason is shown in Fig. 3-14. This shows a calculation we made of the coulomb excitation of ${ }^{239} \mathrm{v}$ by various ions. The dashed line at $1 \%$ probability indicates a probability which can conveniently be seen with present techniques. We already have seen up to the $14^{+}$state in ${ }^{238} \mathrm{v}$ with ${ }^{40} \mathrm{Ar}$. If $\mathrm{kr}$ projectiles are used still higher gtates should be reached, say $20^{+}$or $22^{+}$. With Xe beams still higher spin states in the ground band should be reached and with $\mathrm{Pb}$ beams, states as high as spin $34^{+}$may be excited in ${ }^{238} \mathrm{u}$. These are calculations and not experimental 
results. They are calculated using rigid-rotor matrix elements for ${ }^{238} \mathrm{U}$, and not taking into account any other states, because the program i.s not big enough (20 inputs are all that we can handle in our program). So, these results are not necessarily guaranteed; there will be excitations to other states and that may cut down these yields. Nevertheless, I think there is a reasonable hope that we can approach these spin values with the beams of $\mathrm{Kr}$ and $\mathrm{xe}$ thạt will become available. It will be very exciting if we can just see these states, for if our ideas on (HI, xnY) reactions are correct, it is not likely that they will allow the observation of states nuch higher in spin than the $22 h$ already observed. And just the observation of such high-spin states as unique members of the ground-state band will be a very interesting development for present-day ideas on the nature of the yrast levels and will greatly influence the arguments goiny on about the origin of back-bending. I think there will be exciting developments in these problems.

However, to get quantitative numbers out of this work, there is a real complication. The present-day computers are just not big enough to handle all the data in such a problem, and there are no computer codes big enough. I already described to you what we had to do for calculations up to the $8^{+}$state. If we go, say to the $20^{+}$state, the evaluation of the matrix elements will require a much more efficiently arranged computer code than exists today. In addition, a very large number of matrix elements will have to be measured or estimated, and a way will have to be found to calculate or estimate the guantal corrections. I think we can do the experinents, and even obtain qralitative answers from them. I am not sure how long it may take to get quantitative results. 
We11, an enormous number of experiments can be done in the near future with heavier ions; we have not even touched upon the topic of recoil experiments with heavy ions. Recoil-distance measurements will become easier and more accurate with heavier ions; they will be combined with Doppler-shift attenuation measurements to account for slowing down in the plunger. It should be possible to measure quite accurately, as accurately as stopping-power measurements permit, half-lives in the range of psec and sub-psec, and that is of course very exciting. In addition, I am sure that a number of new, some as yet unknown, topics will come out of the work with very heavy ions, including a solution to the problem of the mechanism of reactions at high angular momentum. 
Table 3-1. Values of $\langle\alpha\rangle$ from $B(E 2\rangle$ and Energy Level Measurements. ${ }^{2}$

\begin{tabular}{lll} 
Nucleus & $10^{3}(\alpha)$ fran B(E2) & $-10^{3}$ B/A fr.jm $0,2,4$ Levels \\
\hline${ }^{166_{\mathrm{Er}}}$ & $-(2.1 \pm 1.0)$ & $0.9 \pm 0.2$ \\
$172_{\mathrm{Yb}}$ & $-(1.3 \pm 0.9)$ & $0.6 \pm 0.1$ \\
$174_{\mathrm{Yb}}^{176_{\mathrm{Yb}}}$ & $-(0.5 \pm 1.0)$ & $0.5 \pm 0.1$ \\
\hline
\end{tabular}

a) R. O. Sayer, P. H. Stelson, F. K. McGowar, W. T. Milner, and R. I. Robinson, Phys. Rev. Cl, 1525 (1970). 
Table 3-2. Average Values of $\alpha$ fcr ${ }^{152} \mathrm{Sm}$.

\begin{tabular}{ll} 
Method & $10^{3}(\alpha)$ \\
\hline Multiple excit. $\left.^{a}\right)$ & $3.4 \pm 1.0$ \\
Multiple excit. $^{\text {b) }}$ & $2.7 \pm 1.7$ \\
Multiple excit. $^{c}$ & $1.7 \pm 1.4$ \\
-B/A from $0,2,4$ Levels & $6.7 \pm 1$ \\
Plunger & $1.9 \pm 0.6$ \\
\hline
\end{tabular}

a) I. A. Fraser, J. S. Greenberg, S. H. Sie, R. G. Stokstad and D. A. Bromley, Phys. Rev. Letters 23 (1970) 1137.

b) R. O. Sayer, P. H. Stelson, F. K. MeGowan, W. T. Milner and R. I. Robinson, Phys. Rev. Cl (1970) 1525.

c) F. S. Stephens, D. Ward, R. M. Diamond, J. deBoer and R. Covello-Moro, unpublished.

d) R. M. Diamond, F. S. Stephens, K. Nakai anä R. Norahagen, Phys. Rev. C3 (1971) 344. 
Table 3-3. Correction (in percent) to Calculated B(E2; I+I-2) of ${ }^{152} \mathrm{Sm}$ from $54 \mathrm{MeV}{ }^{16} \mathrm{o}$ scattered at $160^{\circ}$.

\begin{tabular}{lccc}
\hline \multicolumn{1}{c}{ Cause } & $I=4$ & $I=6$ & $I=8$ \\
\hline \begin{tabular}{l}
\hline -band \\
$Y$-band
\end{tabular} & -1.0 & +1.4 & +0.6 \\
$K=0$ octupole band & +1.5 & +1.8 & +1.8 \\
$K=1$ octupole band & +0.4 & +3.6 & +8.3 \\
E4 moment & +0.9 & -1.2 & -3.6 \\
Total & +3.7 & +10.5 & +19.6 \\
$10^{3} \Delta \alpha$ & +5.5 & +16.1 & +26.7 \\
& +3.7 & +3.2 & +2.6
\end{tabular}


Table 3-4. Calculated population of the $4^{+}$state in ${ }^{152} \mathrm{Sm}$.

\begin{tabular}{|c|c|c|c|c|c|c|}
\hline$I^{\pi^{L}}$ & ${ }_{\mathrm{k}}^{\mathrm{k}}$ & $\begin{array}{c}E \\
\text { (MeV) }\end{array}$ & $\begin{array}{c}B\left(E \lambda ; o^{+}+I^{\pi}\right. \\
\left.e^{2} b^{\lambda}\right)\end{array}$ & $f\left(4^{+}\right)^{a}$ & $\begin{array}{c}E_{\alpha}=1 \\
f\left(4^{+}\right) \sigma_{i k} \\
(\mu b)\end{array}$ & $\begin{array}{l}.38 \mathrm{MeV} \\
f\left(4^{+}\right) d \sigma_{i k} \\
(\mu b)\end{array}$ \\
\hline \multirow[t]{2}{*}{$2^{+}$} & $O(B)$ & 0.811 & 0.023 & 0.21 & 57 & 2.6 \\
\hline & $2(\gamma)$ & 1.087 & 0.083 & 0.013 & 5 & 0.3 \\
\hline \multirow[t]{2}{*}{$3^{-}$} & 0 & 1.042 & 0.14 & 0.30 & 29 & 1.9 \\
\hline & 1 & 1.578 & 0.078 & 0.73 & 9 & 0.5 \\
\hline $6^{+}$ & 0 & 0.7067 & $b$ & 1.60 & 0.6 & 0.14 \\
\hline $4^{+}$ & 0 & 0.3665 & b & 1.00 & 328 & 53.4 \\
\hline \multicolumn{3}{|c|}{ Total } & & & 429 & 58.8 \\
\hline
\end{tabular}
a) Eraction of the decay which goes to the $4^{+}$level.
${ }^{b)}$ Only multiple $\mathrm{E} 2$ excitation is considered here. 
Table 3-5. Reduced Hexadecapole Matrix Elements.

Nucleus

$\left\langle 4^{+} \| \mathrm{M}(\mathrm{E} 4) \mathrm{Ho}^{+}\right\rangle$in $\mathrm{eb}^{2}$

152 Sm

$0.45 \pm 0.09^{\mathrm{a}}$

$0.35 \pm 0.07^{b}$

$0.47 \pm 0.07^{\mathrm{c}}$

$0.37 \pm 0.09^{d}$

${ }^{154} \mathrm{Sm}$

$0.67 \pm 0.08^{\mathrm{e}}$

$0.43 \pm 0.08^{b}$

$0.65 \pm 0.05^{C}$

$0.54 \pm 0.11^{\mathrm{d}}$

${ }^{158} \mathrm{Gd}$

$0.39 \pm 0.11^{\alpha}$

$0.39 \pm 0.09^{c}$

${ }^{160} \mathrm{Gd}$

$0.36 \pm 0.10^{b}$

162 Dy

$0.27 \pm 0.10^{b}$

${ }^{164}$ Dy

$0.28 \pm 0.11^{b}$

$0.25 \pm 0.16^{\mathrm{C}}$

${ }^{166} \mathrm{Er}$

$\begin{aligned} & 0.06+0.12^{b} \\ & 0.12=0.18 \\ & 0.18^{c}\end{aligned}$

${ }^{168}$ Er

$\begin{aligned} 0.20 & +0.12^{b} \\ & =0.18\end{aligned}$

$0.12 \pm 0.20^{\circ}$

$170_{\text {Ex }}$

$0.24+0.14^{b}$

174.

$0.23 \pm 0.17^{c}$

230 Th

$1.04 \pm 0.21^{\mp}$

232 Th

$1.60 \pm 0.27^{9}$

$234 \mathrm{v}$

$1.70 \pm 0.10^{g}$

236

$1.23 \pm 0.28^{g}$

238

$1.12 \pm 0.23^{\mp}$

238 Pu

$1.45 \pm 0.21^{9}$

${ }^{240} \mathrm{Pu}$

$1.18 \pm 0.22^{9}$ 
Table 3-5. (cont.)

Nucleus $\quad\left\langle 4^{+} \|_{M(E 4)} 10^{+}\right\rangle$in $\mathrm{eb}^{2}$

\begin{tabular}{|c|c|c|}
\hline${ }^{242} \mathrm{Pu}$ & 0.70 & $\begin{array}{l}+0.3^{9} \\
-0.4\end{array}$ \\
\hline${ }^{244} \mathrm{Pu}$ & 0.0 & $\begin{array}{l}+0.079 \\
-0.6\end{array}$ \\
\hline${ }_{246}^{244} \mathrm{~cm}$ & -0.4 & $\pm 0.4^{9}$ \\
\hline${ }^{246} \mathrm{Cm}$ & $\begin{array}{r}-0.4 \\
0.0\end{array}$ & $\begin{array}{l} \pm 0.4^{g} \\
+0.4^{9} \\
-0.6\end{array}$ \\
\hline
\end{tabular}

a) F. S. Stephens, R. M. Diamond, N. K. Glendenning and J. deBoer, Phys. Rev. Letters $\underline{24}$ (1970) 1137 .

b) J. X. Saladin, K。 $A_{0}$ Erb, J, E. Holden, I, $Y$. Lee and $T . K$. Saylor, presented at this conference $\mathrm{V}-20$.

C) J. S. Greenberg and A. H. Shaw, presented at this conference V.b.

d) H. Bruckner, D. Pelte, B. Powh, U. Smilansky and K. Traxel, presented at this conference $\mathrm{V}-2 \mathrm{l}$.

e) F. S. Stephens, R. M. Diamond and J. deBoer, Phys. Rev. Letters 27 (1971) 1151. E) F. K. McGowan, C. E. Bemis, Jr., J. I. C. Ford, Jr., W. T. Milner, R. I. Robinson and P. H. Stelson, Phys. Rev. Letters 27 (1971) 1741. g) F. Ko McGowan and P. H. Stelson, private communication, 1972. 
Table 3-6. Quadrupole Moments in ${ }^{114} \mathrm{Cd}$

$$
B(E 2 ; 0+2) \text { in } e^{2} b^{2} \quad Q_{2+} \text { in eb }
$$

$$
\begin{array}{ll}
0.51 \pm 0.02 & -0.70 \pm 0.21^{\mathrm{a})} \\
& -0.6 \pm 0.2^{\mathrm{b}} \\
0.561 \pm 0.005 & -0.85 \pm 0.15^{\mathrm{c})} \\
0.48 \pm 0.05 & -0.49 \pm 0.25^{\mathrm{d})} \\
0.509 \pm 0.009 & +0.05 \pm 0.27^{\mathrm{e})} \\
0.561 \pm 0.017 & -0.68 \pm 0.09^{\mathrm{fl}} \\
& -0.64 \pm 0.19^{\mathrm{g})} \\
0.498 \pm 0.027 & -0.53 \pm 0.17^{\mathrm{h})} \\
0.513 \pm 0.005 & -0.40 \pm 0.12^{\mathrm{i})} \\
& -0.28 \pm 0.09^{j)} \\
& -0.35 \pm 0.07^{\mathrm{k})}
\end{array}
$$

a) J. deBoer, R。 G. Stokstad, G. D. Symons and A. Winther, Phys. Rev. Letters 14 (1965) 564 .

b) P. Ho Stelson, W. T. Milner, J. L. C. Ford, Jr., F. K. McGowen and R. L. Robinson, Bull. Amer. Phys. Soc. 10 (1965) 427.

c) R. G. Stokstad, I. Hall, G. D. Symons and J. deBoer, Nuel. Phys. A92 (1967) 319. d) J. S. Simpson, D. Eccleshall, M. J. L. Yates and N. J. Ereeman, Nucl. Phys. 492 (1967) 177.

e) J. S. Simpson, D. Smilansky and J. P. Wurm, Phys. Letters $27 \mathrm{~B}$ (1968) 633.

f) J. X. Saladin, J. E. Glenn and R. J. Pryor, Phys. Rev. 186 (1969) 1241;

J. E. Glenn and J. X. Saladin, Phys. Rev. Letters 19 (1967) 33.

g) G. Schilling, R. P. Schazenberg and J. W. Tippie, Phys. Rev. Letters 19 (1967) 318; Phys. Rev. Cl. (I970) 1400. .

h) D. S. Andreyev, G. M. Gusinsky, K. I. Erokhina, M. F. Kudojarov, I. Kh. Lemberg and I. V. Chugunov, Phys. Letters 32B (1970) 187. 
Table 3-6 (cont.)

i) A. M. Kleinfeld, J. D. Rogers, J. Gastebois, S. G. Steadman and J. deBoer, Nucl. Phys. Al58 (1970) 81.

j) z. Berant, R. A. Eisenstein, J. S. Greenberg, Y. Horowitz, U. Smilansky,

P. M. Tandon, A. M. Kleinfeld and H. G. Mäggi, Phys. Rev. Letters 27 (1971) 110.

k) R. O. Larsen, J. A. Thomson, R. G. Kerr, R. P. Scharenberg and W. R. Lutz, prlvate communication, 1972. 
Part III

Eigure Captions

Fig. 3-1. The Ruther zord scattering of (point) projectile $z_{1}$ e on target nucleus $\mathrm{z}_{2}$ e. The target nus:leus has a quadrupole deformation.

Fig. 3-2. Plots of $R_{\text {exp }} / R_{c o m p}$ for ${ }^{114} \mathrm{Cd}$ vs. incident projectile energy (a) for ${ }^{4} \mathrm{He}$ ions, and (b) for ${ }^{16} \mathrm{o}$ ions; $\mathrm{R}=\mathrm{d \sigma}\left(2^{+}\right) /\left(\mathrm{d \sigma}(\right.$ elastic $\left.)+\operatorname{d\sigma }\left(2^{+}\right)\right)$.

(Taken from Z. Berant, R. A. Eisenstein, J. S. Greenberg, Y. Horowitz, U. Smilansky, P. N. Tandon, A. M. Kleinfeld, and H. G. Mäggi, Phys. Rev. Letters 27, 110 (1971), courtesy of Dr. J. S. Greenberg.)

Fig. 3-3. Effective values of the quadrupole moment of ${ }^{48} \mathrm{Ti},{ }^{56} \mathrm{Fe}$, and ${ }^{60} \mathrm{Ni}$ as a function of the projectile bombarding energy expressed ir terms of the parameter $t$ of Eq. (3-8). (Taken from P. M. S. Lesser, D. Cline, R. Goode, and R. N. Horoshko, Nucl. Phys. A190, 593 (1972), courtesy of Dr. D. Cline.)

Fig. 3-4. The levels of ${ }^{152} \mathrm{sm}$ used in calculating the reauced transition probabilities of the ground band. Levels whose energies are given in parentheses are estimated.

Fig. 3-5. Ganma-ray spectra taken in coincidence with back-scattered ${ }^{16} 0$ (upper) and 40 Ar (lower) projectiles coulomb exciting ${ }^{152} \mathrm{sm}$. Fig. 3-6. Coulamb excitation of a $4^{+}$state in the ground band of a doublyeven nucleus by (a) double-E2 excitation, (b) E4 excitation, (c) interference between (a) and (b).

Fig. 3-7. Relationship between reduced E4 matrix element and a) the back-scatter cross section for $10.4 \mathrm{MeV}{ }^{4} \mathrm{He}$ ions normalized to the case of zero Es matrix element and $b)$ the deformation parameter, $B_{4}$, using a radius of $R_{0}=1.2 \mathrm{~A}^{1 / 3}$ 血 and $a \beta_{2}$ which yields the experinental E2 moment. 
Fig. 3-8. Gamma-ray spectrum taken in coincidence with $10.4 \mathrm{MeV}$ back-scattered 4 He on natural sm target.

Fig. 3-9. Ratio of experimental to calculated (semi-classical, no E4) cross sections for exciting the $4^{+}$level of ${ }^{152} \mathrm{Sm}$ vs. the ${ }^{4}$ He energy. The solid points are for an enriched ${ }^{152} \mathrm{sm}$ target and the open ones for a natural Sm target. The triangles and circles are back-scatter and singles results, respectively. The dashed and solid lines are the calculated results for back-scatter soincidences and singles, respectively, with $\left(O^{+} \mathrm{M}(\mathrm{E} 4) \mathrm{H4^{+ }}\right)=+0.35 \mathrm{eb}^{2}$.

Fig. 3-10. The shape of ${ }^{154}$ sm indicated by the values of $\beta_{2}$ and $\beta_{4}$ given in Table 3-5, ref. (e).

Fig. 3-11. a) Illustration of interference between lst order excitation of $2^{+}$ state and 2nd order excitation, from which "reorientation process" obtains name. b) Schematic of lst order excitation, reorientation effect, interference with higher-lying $2^{+\prime}$ state, and interference by higher-lying $1^{-}$state (for example, virtual excitation through the giant dipole state). Fig. 3-12. Plot of $\mathrm{Q}_{2}$ for nuclei around neutron number 82: Ba (J. S. Simpson, D. Eccleshall, M. J. I. Yates and N. J. Ereeman, Mucl. Phys. A94 (1967) 177; J. R. Kerns, J. X. Saladin, R. J. Ryyor and S. A. Lane, Bull. Amer. Phys. Soc. 14 (1969) 122 ; C. พ. Towsley, R. Cook, D. Cline and R. N. Horoshko, presented at this conference $v-17$ ); Nd (P. A. Crowley, J. R. Kerns and J. X. Saladin, Phys. Rev. C3 (1971) 2049; H. S. Gertzman, D. Cline, H. E. Gove and P. M. S. Iesser, Nucl. Phys. Al51 (1970) 282); and sm (J. S. Simpson, D. Fxcleshall, M. J. I. Yates and N. J. Freeman, Nucl. Phys. A94 (1967) 177; H. S. Gertzman, D. Cline, H. E. Gove, P. M. S. Lesser and J. J. Schwartz, Nucl. Phys. AI51 (1970) 273; G. Goldring and U. Smilansky, 
Phys. Letters 16 (1965) 151; D. Cline, P. Jennens, C. W. Towsley and H. S. Gertzman, presented at this conference V-1E; G. Kaspar, W. Krupfer, W. Ebert and w. R. Hering (North-Holland Publishing Co., Amsterdam) p. 471). Fig. 3-13. Plot of $Q_{2^{+}}$vs. neutron number for Os (S. $A$, Lane and J. X. Saladin, Bull. Amer. Phys. Soc. 16 (1971) 1157; R. J. Pryor and J. X. Saladin, Phys. Rev. Cl (1970) 1573) and Pt (J. E. Glenn, R. J. Pryor and J. X. Saladin, Ehys. Rev. 188 (1969) 1905).

rig. 3-14. Probability of exciting the level of spin I in ${ }^{238} \mathrm{U}$ vs. I for a number of heavy ions, as calculated by the deBoer-winther Coulomb excitation program, using only ground-band levels connected by rigid-rotor E2 matrix alements. 


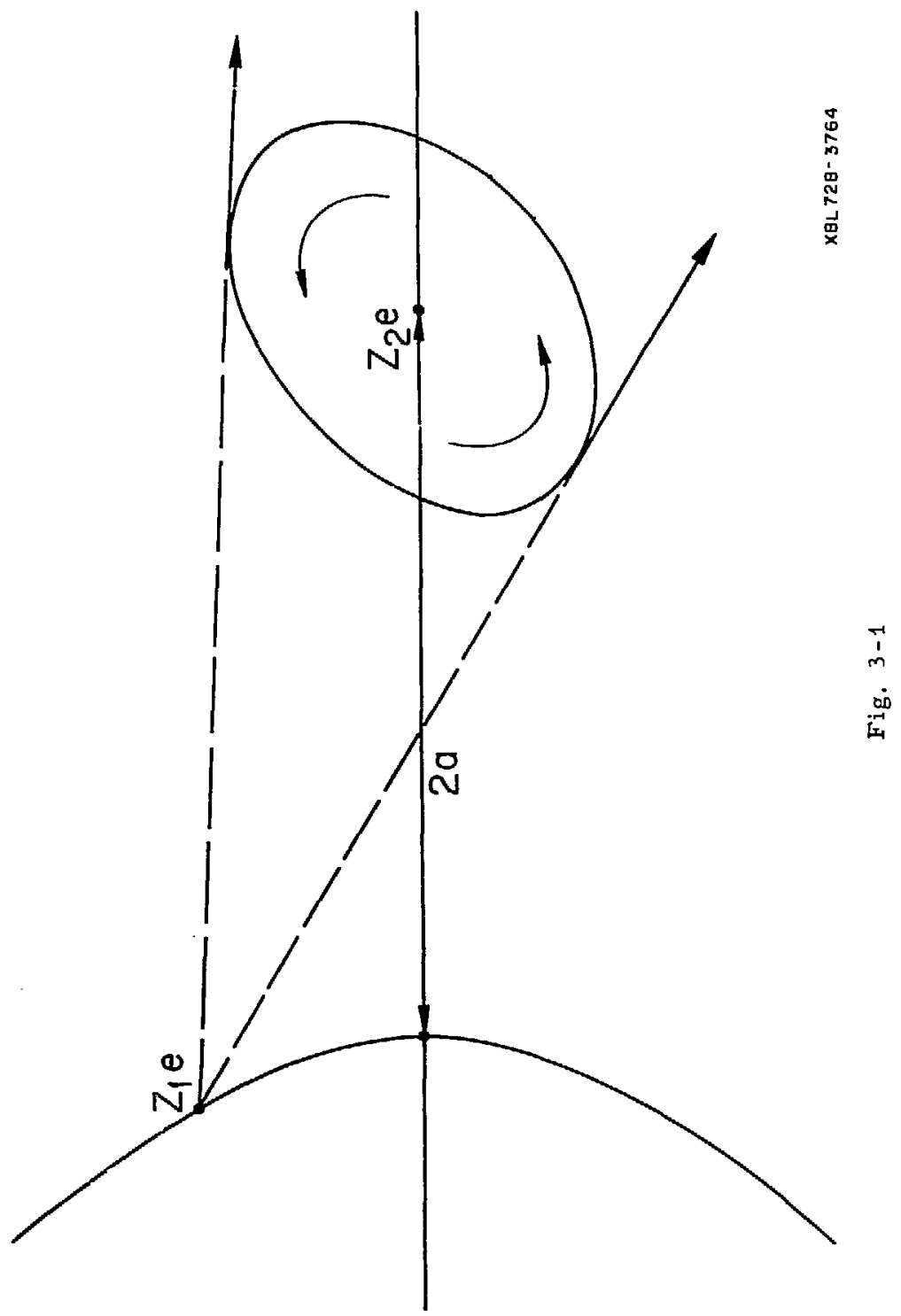



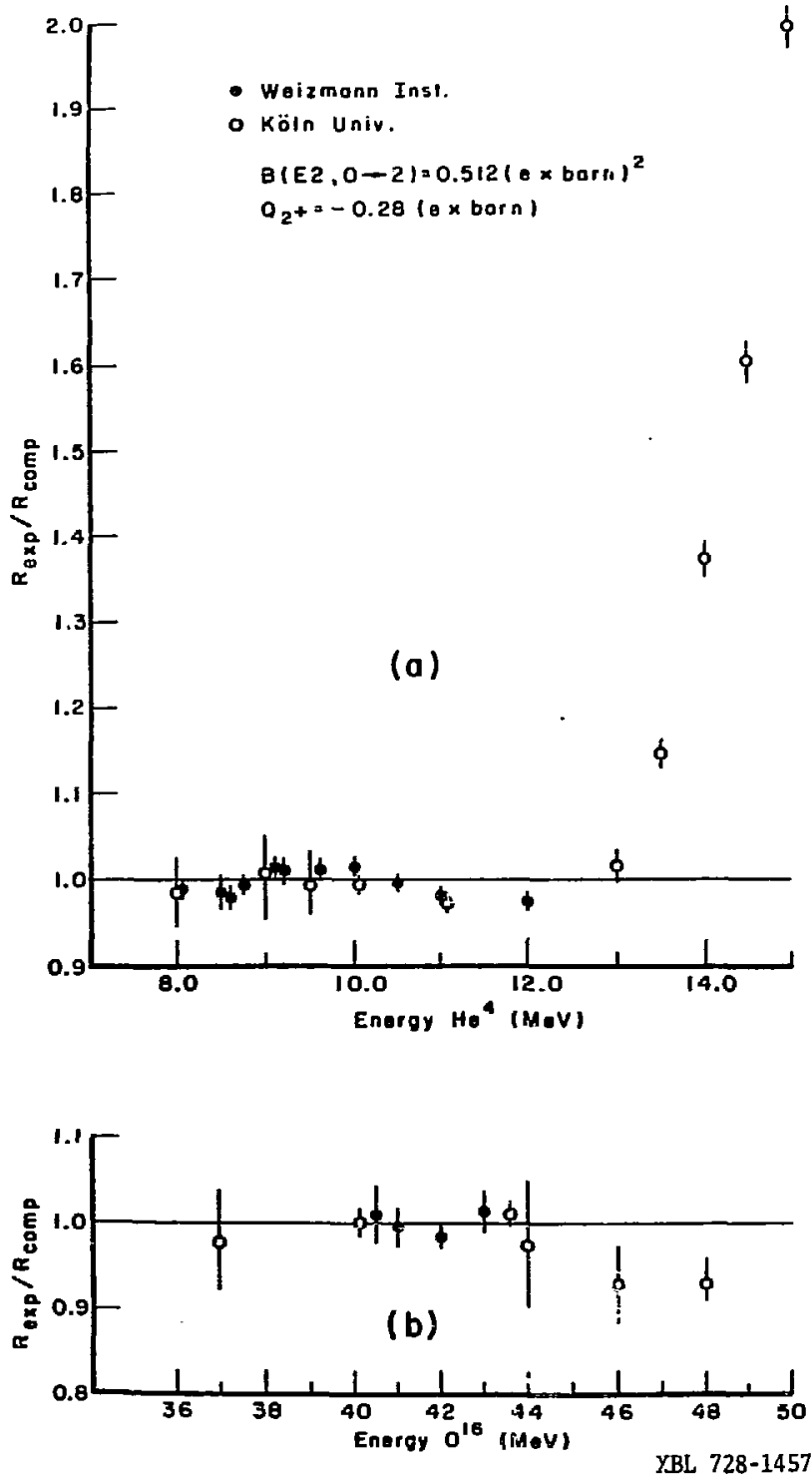

Fic. 3-2 


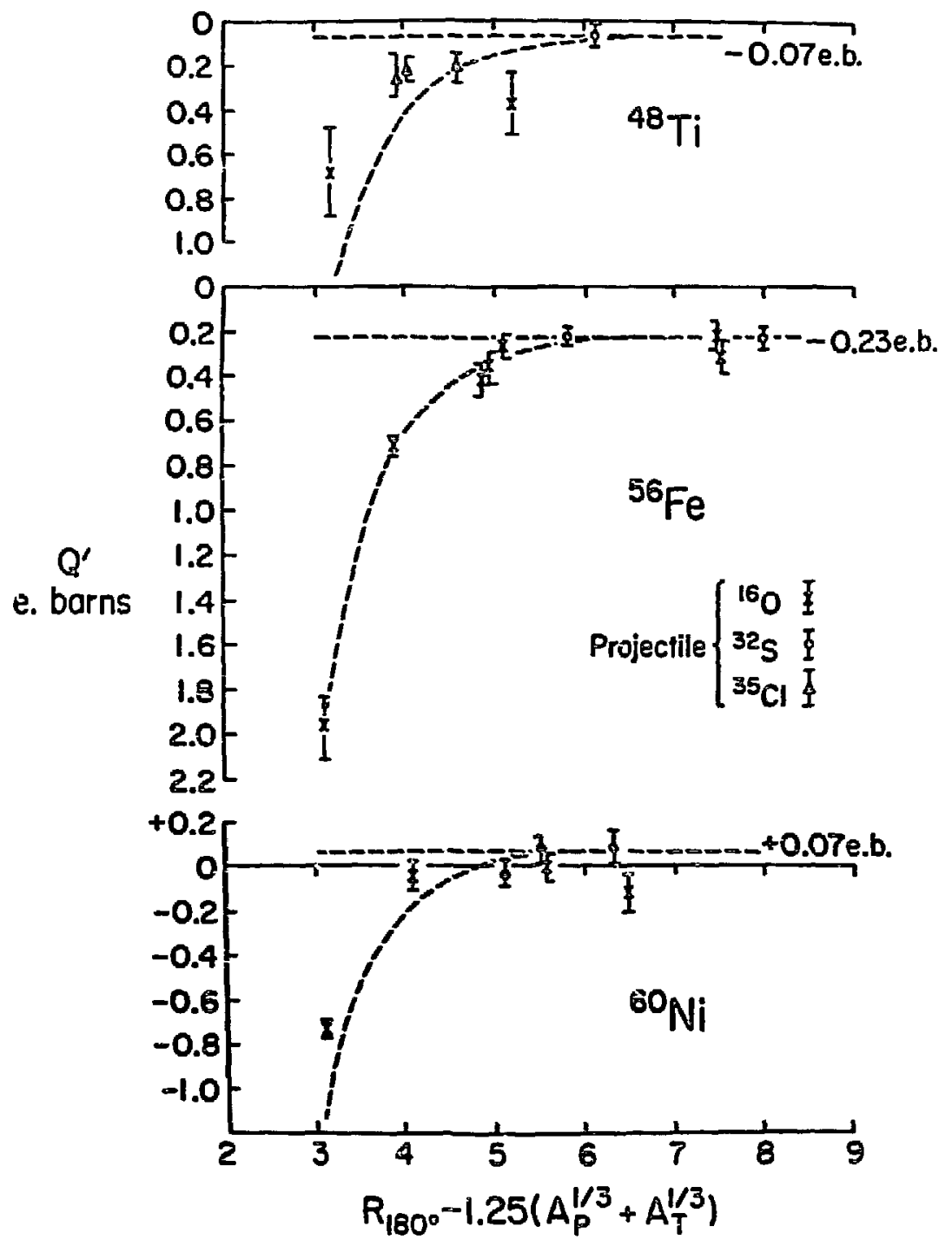

XBL $741-128$ 
$12+-(3100)$

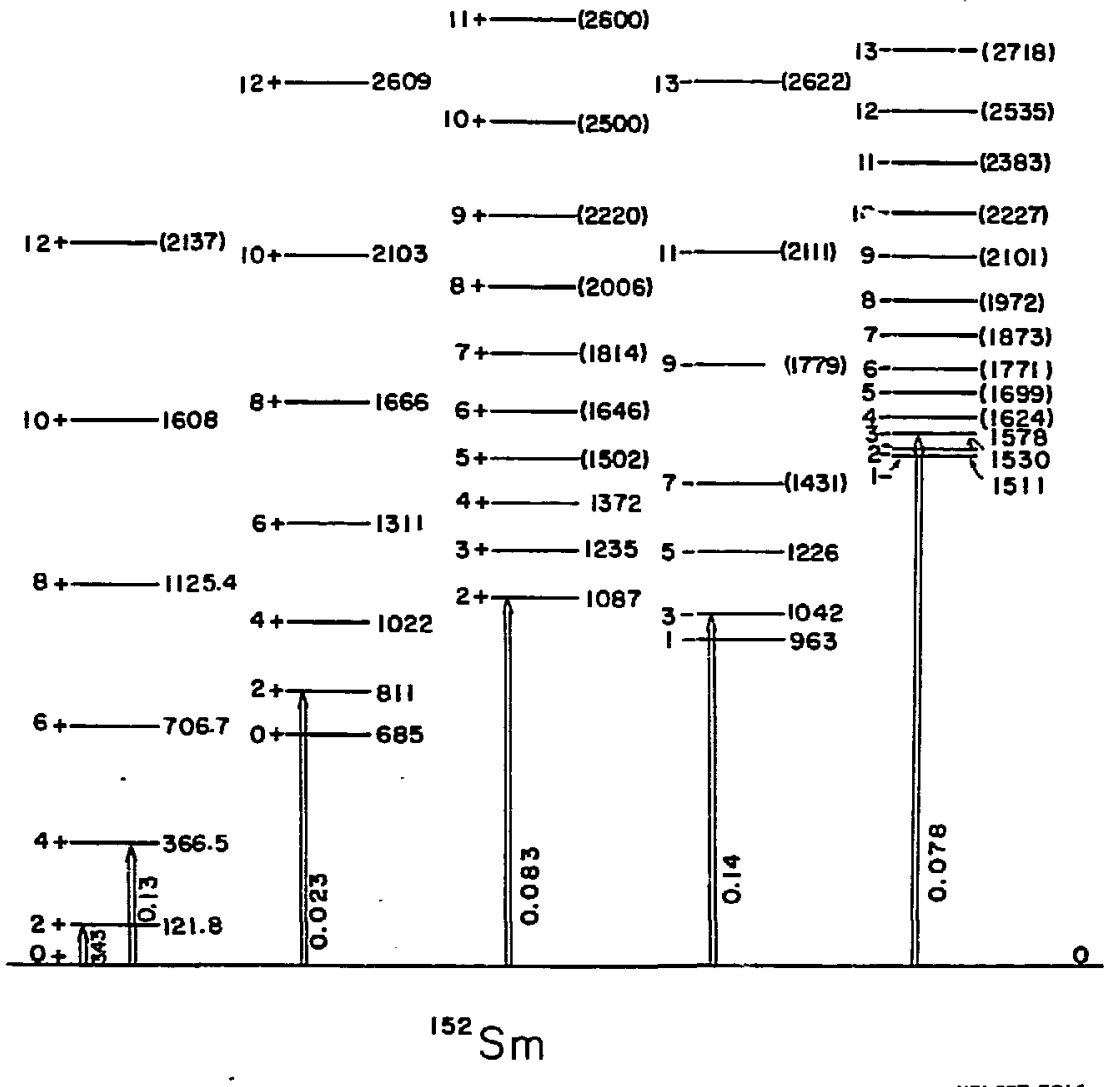

XEL697-3214

Fig. 3-4 


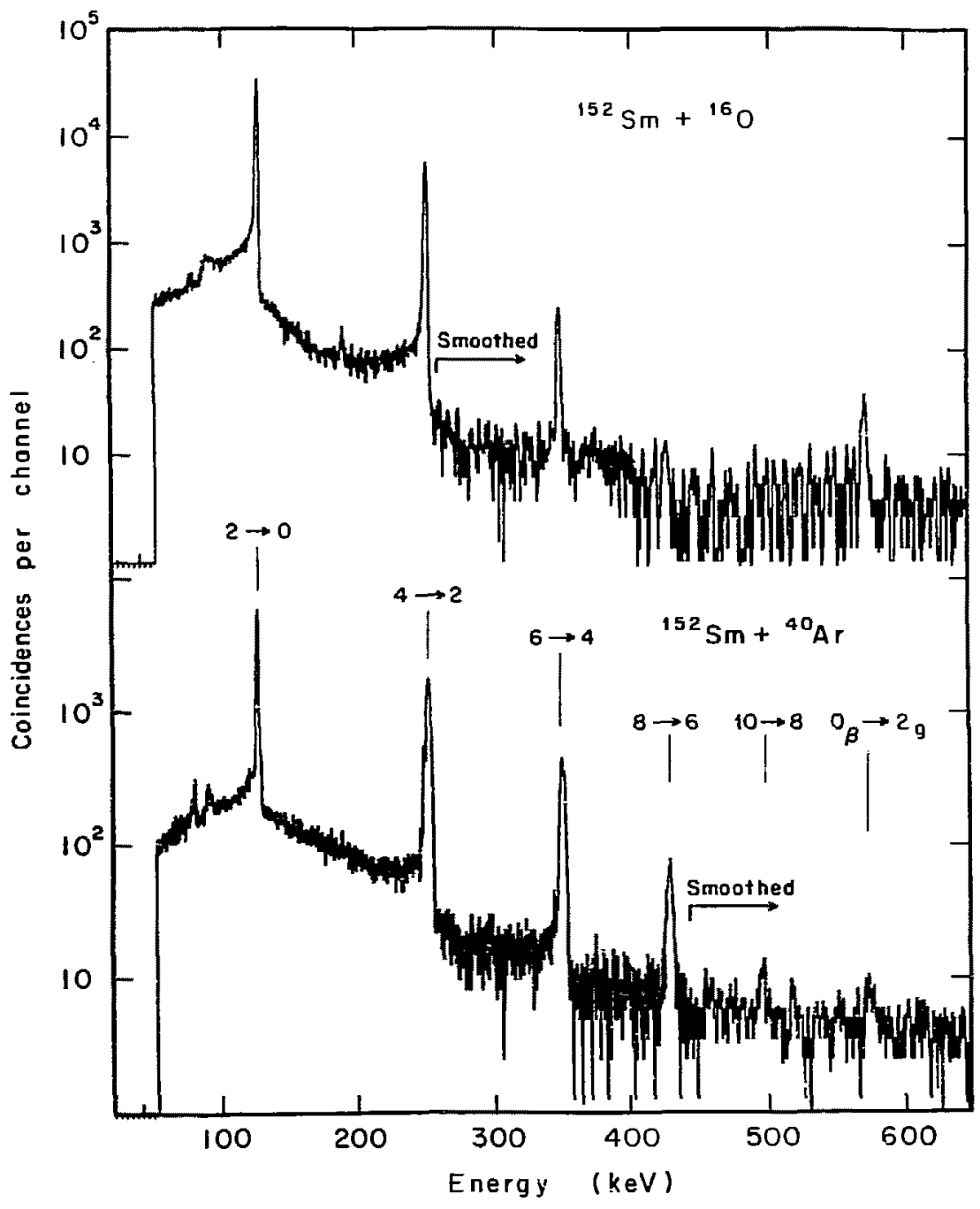

XBL696-3010

Eig. 3-5 


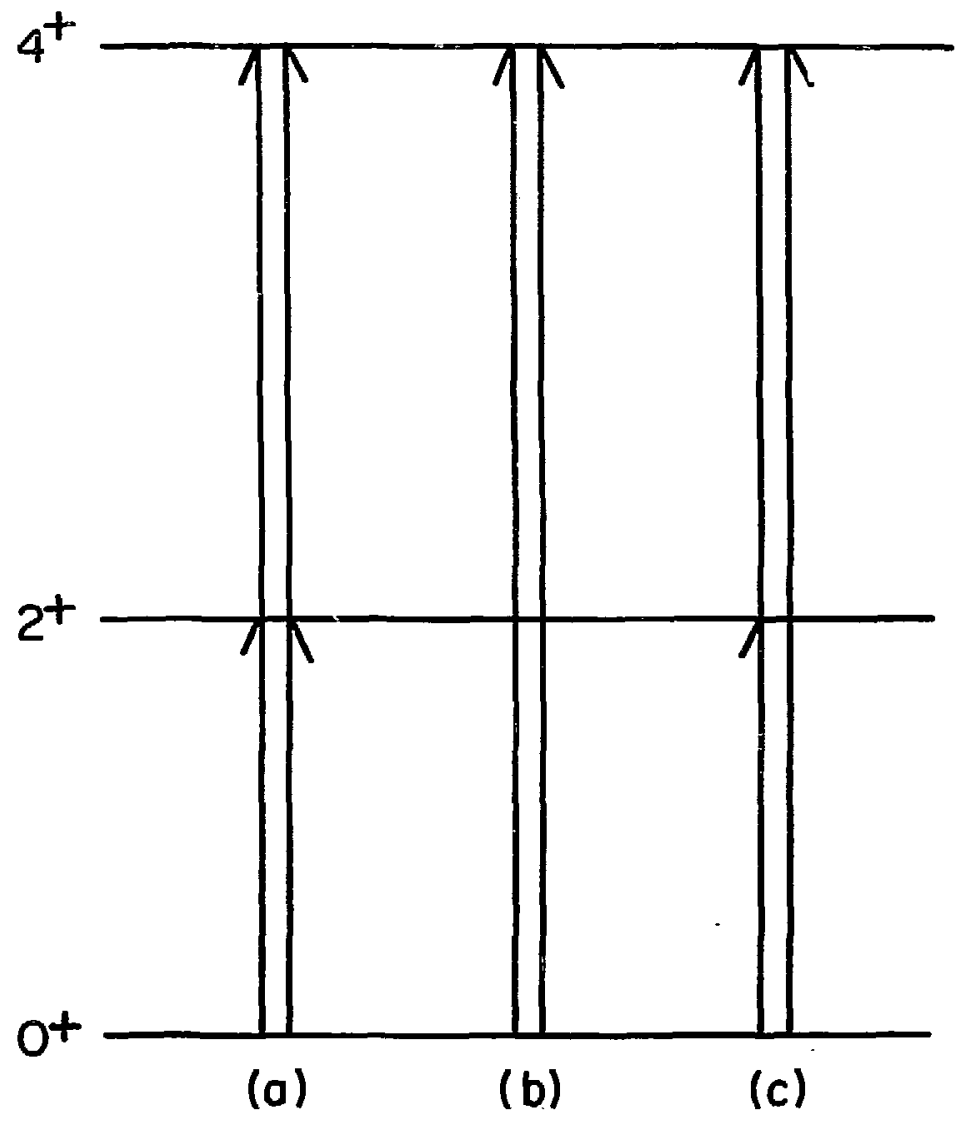

XBL741-2174

Fig. 3-6 


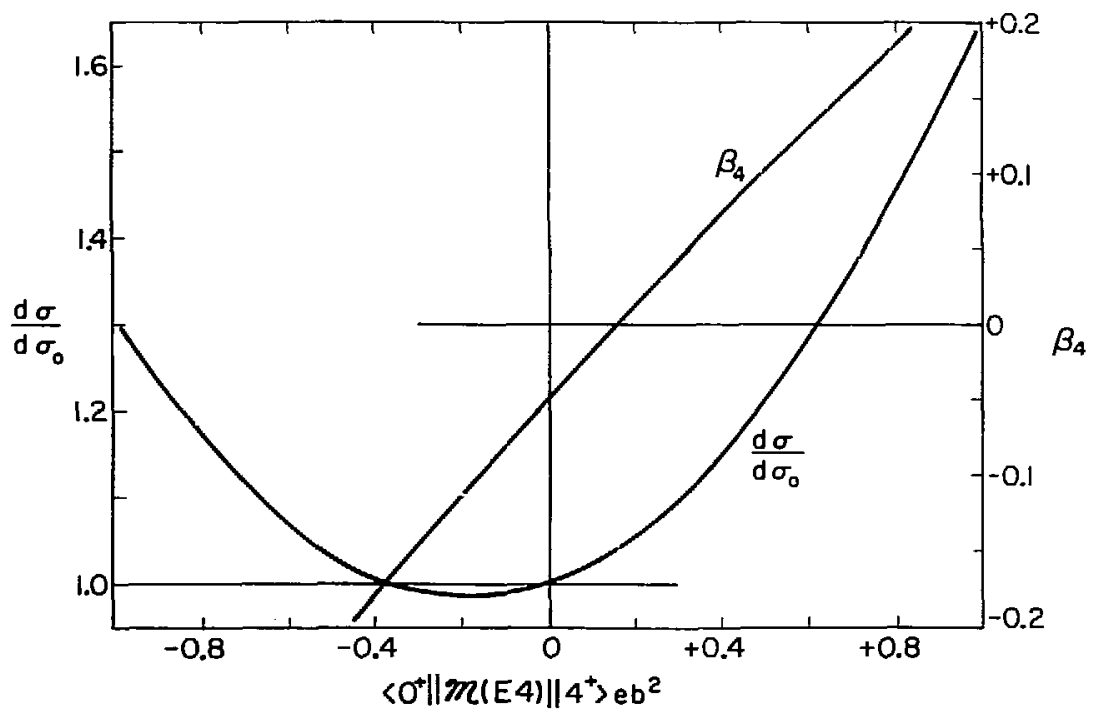

X8L703-2495

Fig. 3-7 


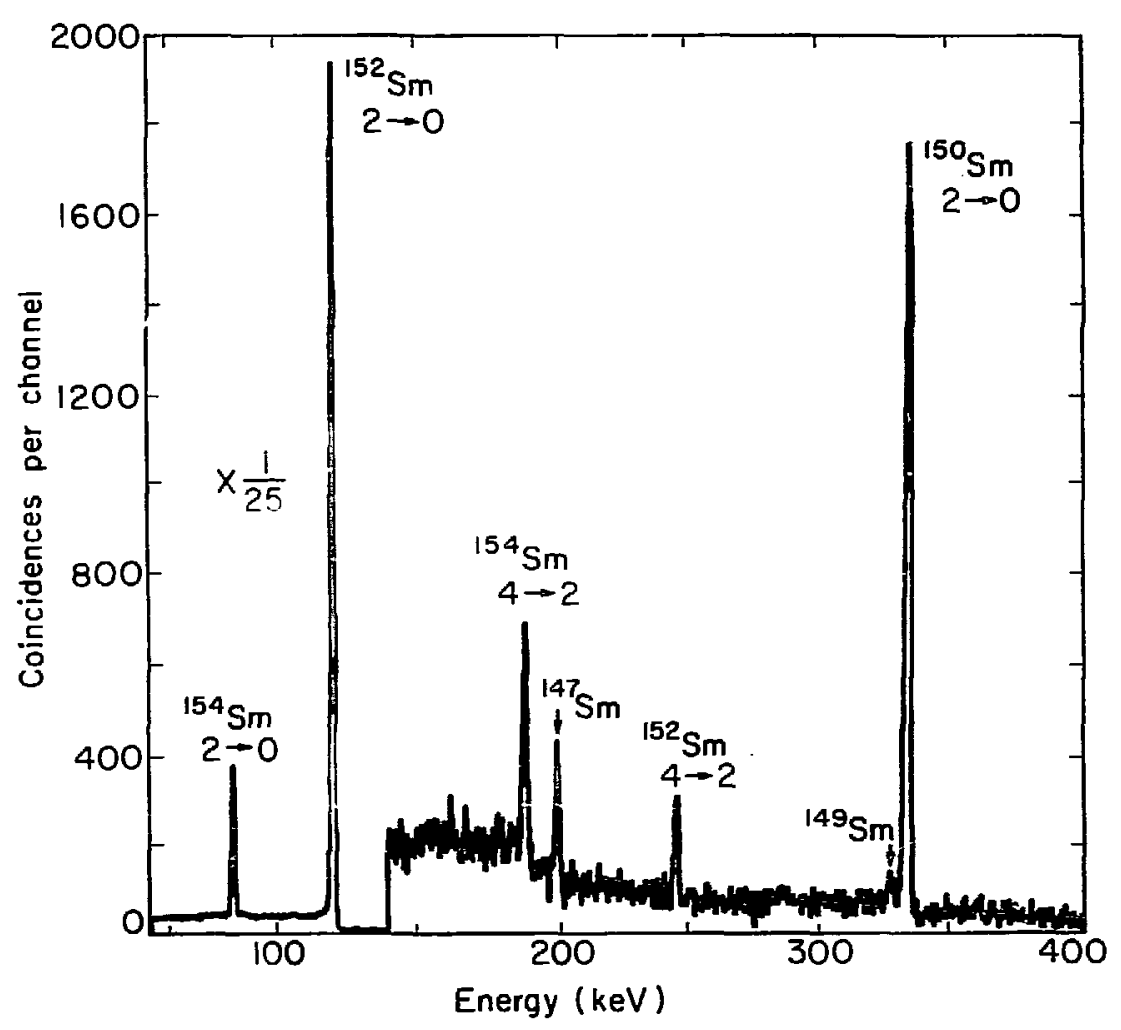

XBL703-2497

Fig. 3-B 


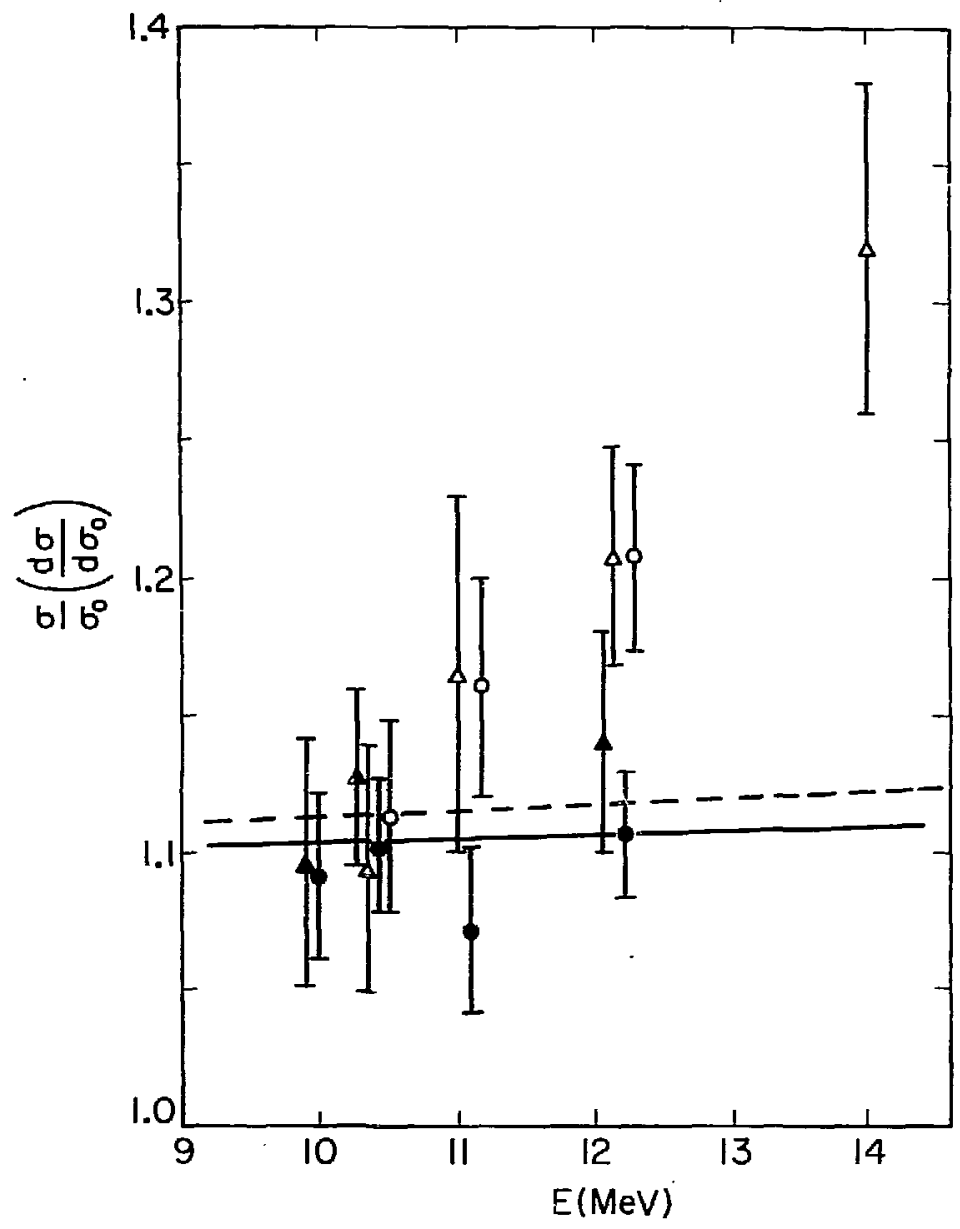

XBL703-2495

Fig. 3-9 


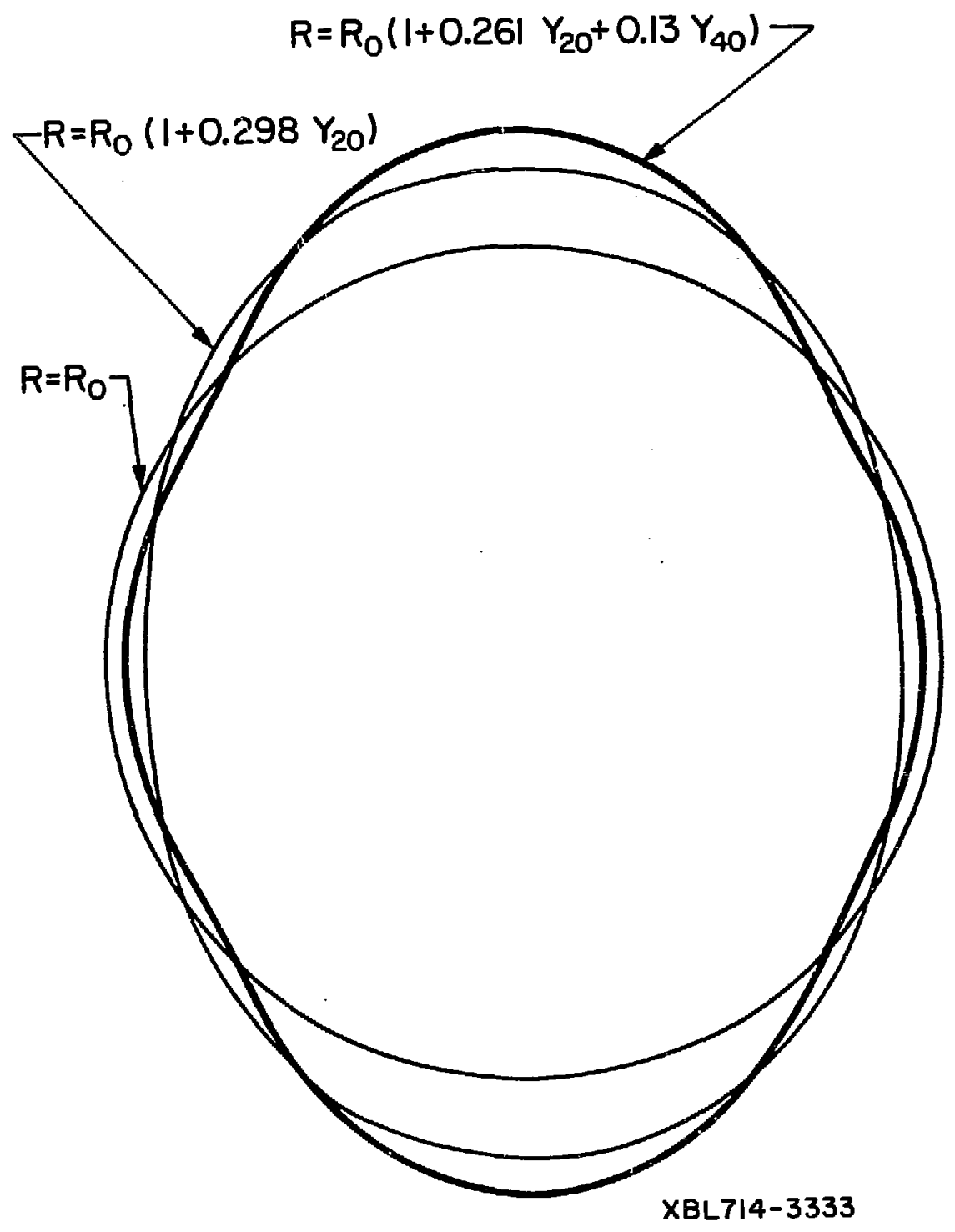

Fig. 3-10 

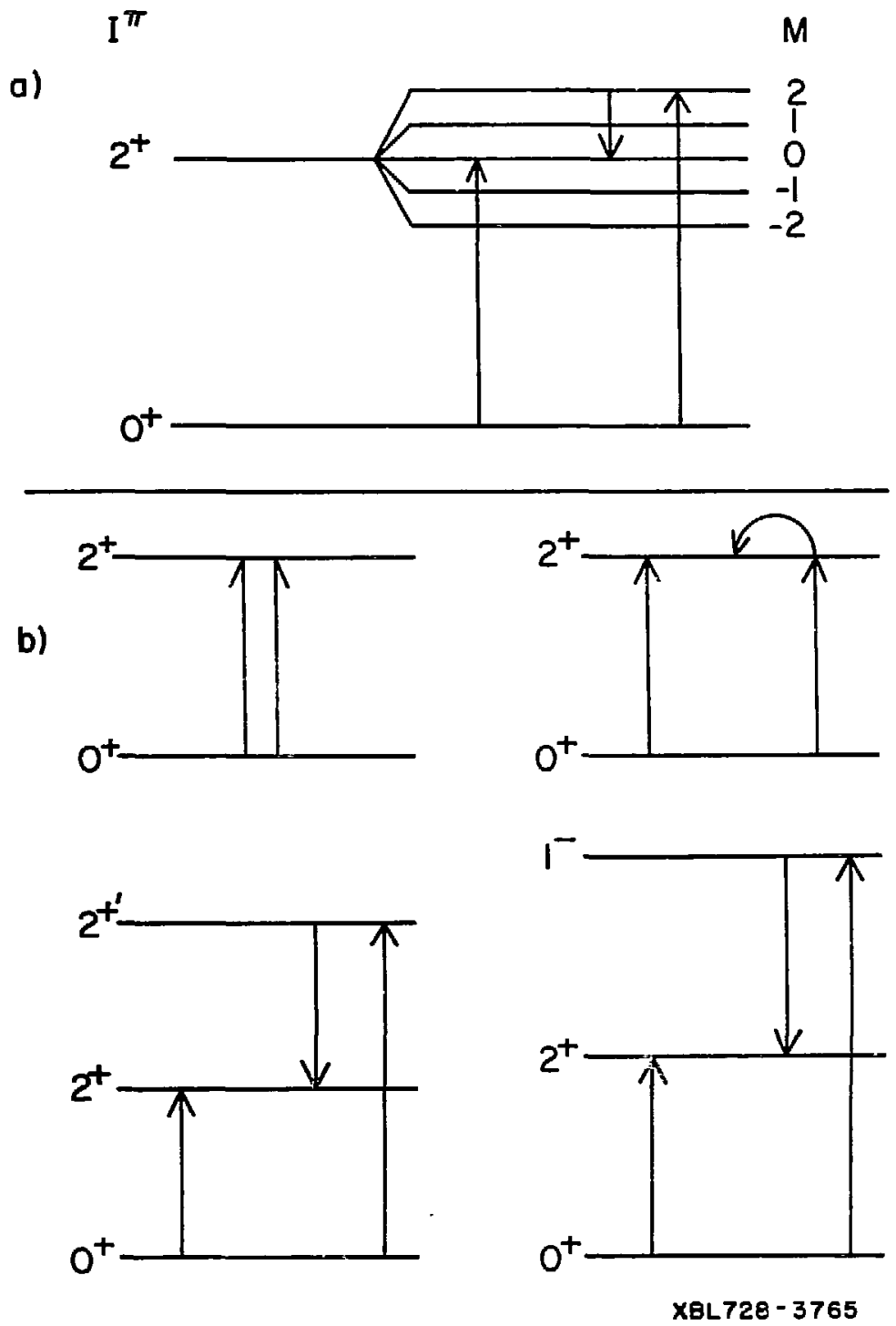

Fig. 3-11 


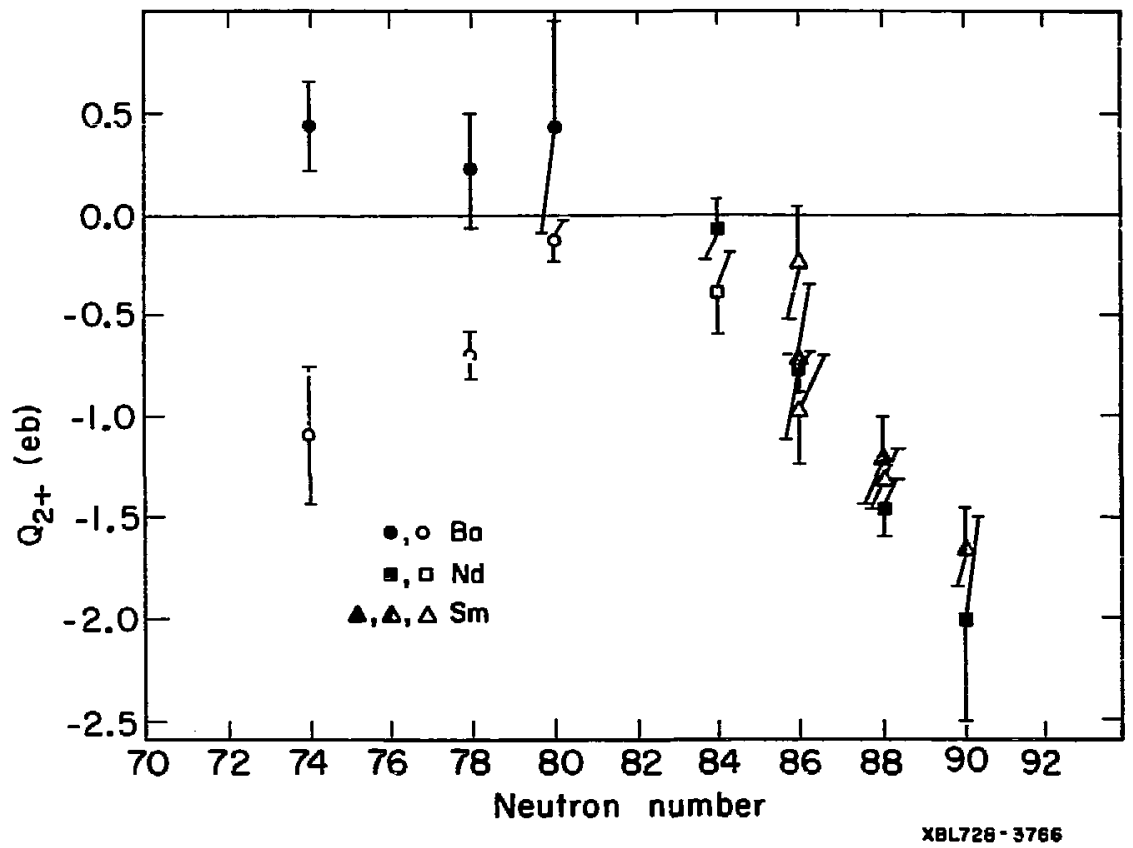

Fig. 3-12 


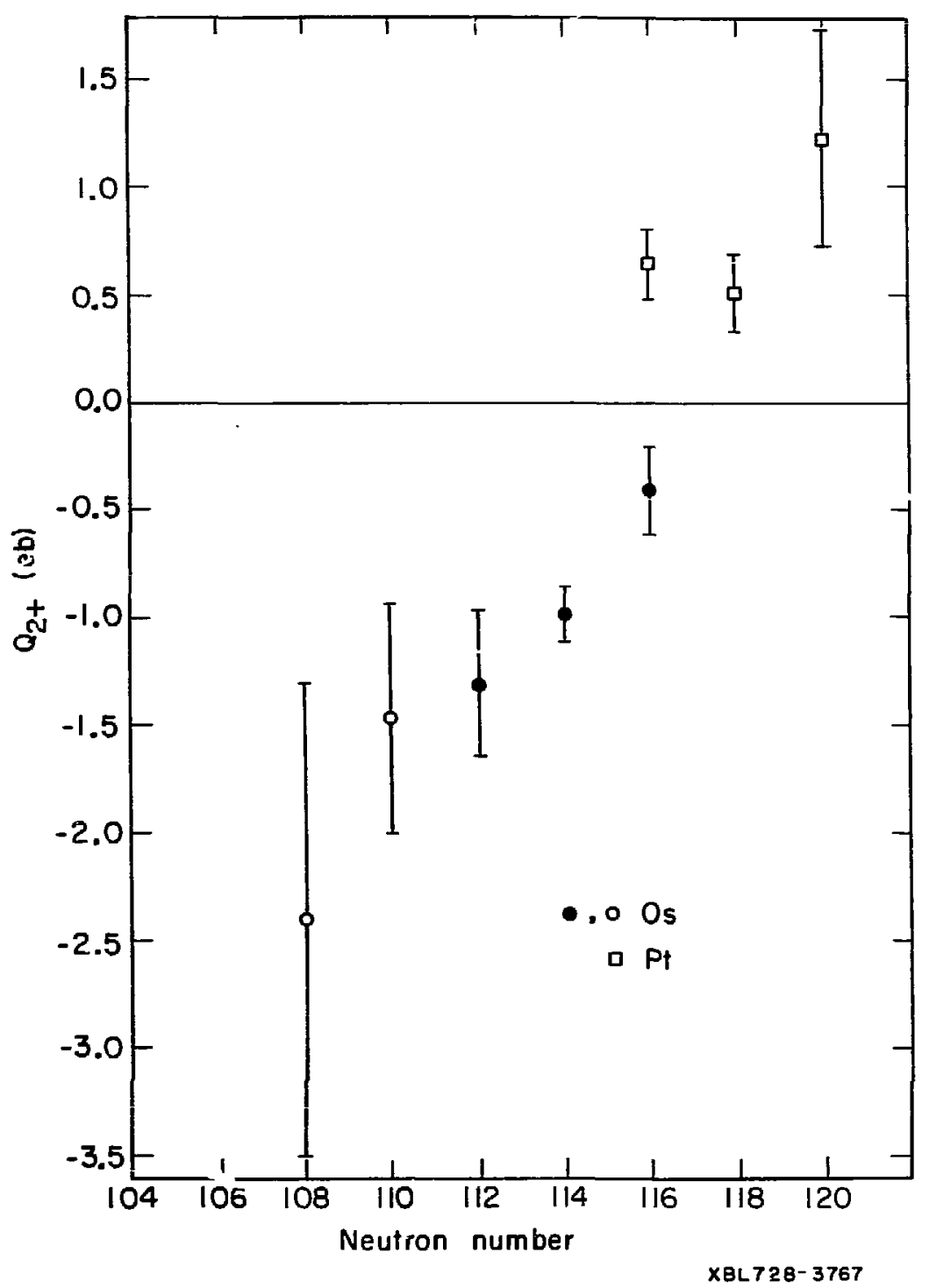

Fig. 3-13 


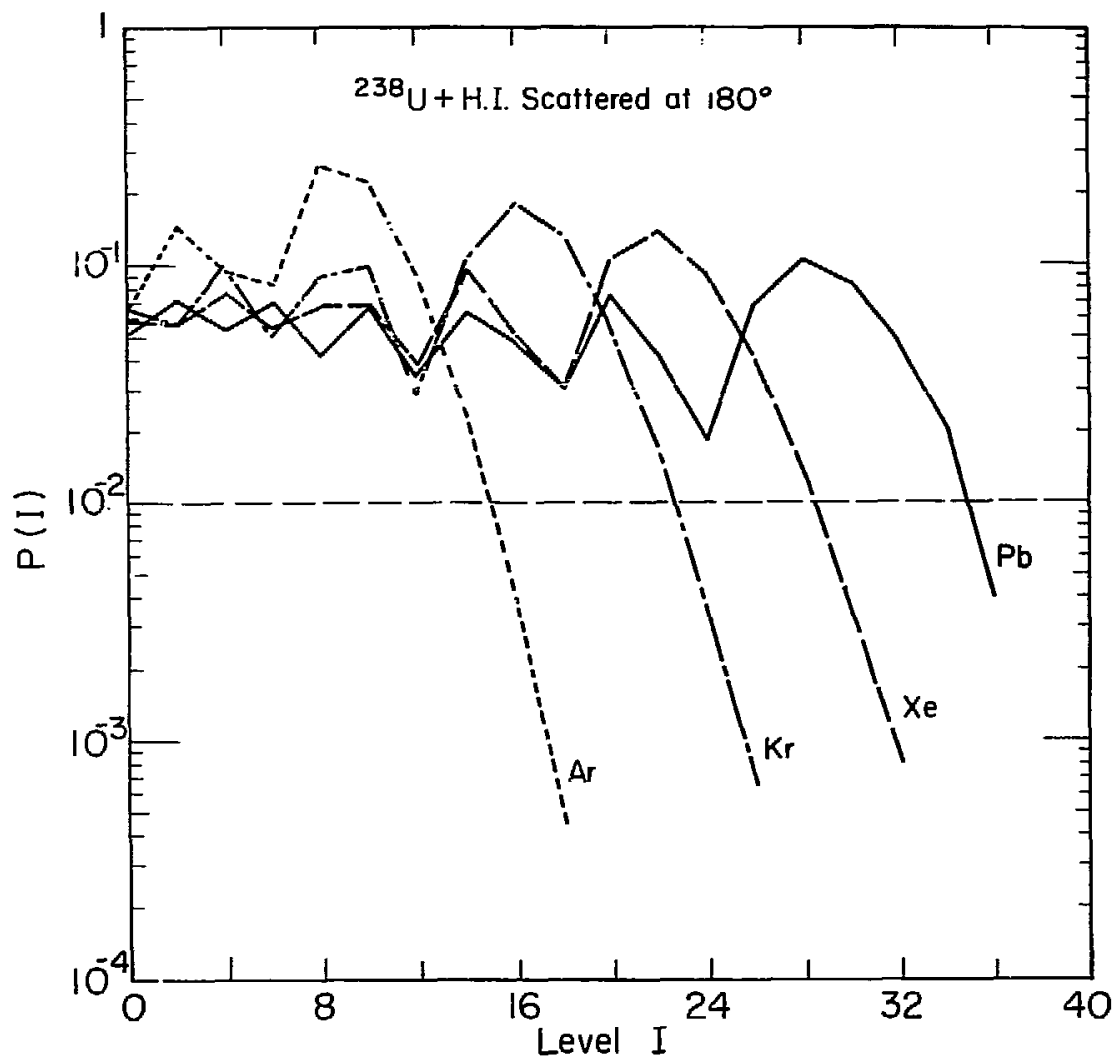

XBL728-3763

Fig. 3-14 